\title{
Solution-state preorganization of cyclic $\beta$-hairpin ligands determines binding mechanism and affinities for MDM2
}

\author{
Yunhui Ge, ${ }^{\dagger}$ Si Zhang, ${ }^{\dagger}$ Mate Erdelyi, ${ }^{\ddagger}$ and Vincent A. Voelz ${ }^{*, \dagger}$ \\ $\dagger$ Department of Chemistry, Temple University, Philadelphia, PA 19122, USA \\ $\ddagger$ Department of Chemistry, Uppsala University, Uppsala, Sweden \\ E-mail: voelz@temple.edu
}

\begin{abstract}
Understanding mechanisms of protein folding and binding is crucial to designing their molecular function. Molecular dynamics (MD) simulations and Markov State Model (MSM) approaches provide a powerful way to understand complex conformational change that occurs over long timescales. Consideration of such dynamics are important for the design of therapeutic peptidomimetic ligands, whose affinity and binding mechanism are dictated by a combination of folding and binding. To examine the role of preorganization in peptide binding to protein targets, we performed massively parallel explicit-solvent MD simulations of cyclic $\beta$-hairpin ligands designed to disrupt the MDM2-p53 interaction. MSM analysis of over $3 \mathrm{~ms}$ of aggregate trajectory data enabled us to build a detailed mechanistic model of coupled folding and binding of four cyclic peptides which we compare to experimental binding affinities and rates. The results show a striking relationship between the relative preorganization of each ligand in solution and its affinity for MDM2. Specifically, changes in
\end{abstract}


peptide conformational populations predicted by the MSMs suggest that entropy loss upon binding is the main factor influencing affinity. The MSMs also enable detailed examination of non-native interactions which lead to misfolded states, and comparison of structural ensembles with experimental NMR measurements. In contrast to an MSM study of p53 TAD binding to MDM2, MSMs of cyclic $\beta$-hairpin binding show a conformational selection mechanism. Finally, we make progress towards predicting accurate off-rates of cyclic peptides using multiensemble Markov models (MEMMs) constructed from unbiased and biased simulated trajectories.

\section{INTRODUCTION}

Understanding the conformational dynamics that underlies protein folding and binding is crucial to designing their molecular function. Molecular dynamics (MD) simulations have steadily become a powerful tool to gain mechanistic understanding of complex processes at the atomic level. Thanks to advances in computer hardware and enhanced sampling strategies, MD simulations can now routinely model conformational dynamics on the microsecond timescale, which is crucial for understanding the significant conformational rearrangements that peptides can undergo, and their association with protein targets.

Cyclic peptides have many beneficial properties for therapeutic development over their linear counterparts, including improved physical and chemical stability, binding affinity and membrane permeability. Much of these benefits are believed to arise through their intrinsic conformational preorganization in solution. 1] 6

A well-studied protein-peptide interaction targeted by cyclic peptides is between the E3 ubiquitin ligase MDM2 (mouse double minute 2 homolog) and the tumor suppressor protein p53, a potent transcription factor which plays a multifaceted role in the cell cycle. ${ }^{7}$ The level and activity of wild-type p53 in the cell is controlled by MDM2, its negative regulator, 11 both by binding to the transactivation domain (TAD) of p53 and inhibiting 
its transcriptional activity, ${ }^{12}$ and by targeting p 53 for degradation in the proteasome. ${ }^{\frac{13}{13}} \mathrm{In}$ many cancers (about 50\%), wild-type p53 is retained, but cannot mediate tumor suppression because of over-expression of MDM2. Therefore, many cancer therapeutic peptidomimetics have been developed to disrupt the MDM2/p53 interaction by competitively binding to MDM2.

The rational design of peptidomimetic ligands targeting MDM2 has benefited greatly from the existence of a crystal structure of p53 TAD (residues 15-29) bound to MDM2, 12 which shows the intrinsically disordered p53 TAD ${ }^{14}$ bound to the hydrophobic cleft of MDM2 in a helical conformation, with p53 TAD residues Phe19, Trp23 and Leu26 filling the cleft. In addition to small molecule binders,,$\frac{1516}{2}$ a number of linear and cyclic and binders of MDM2

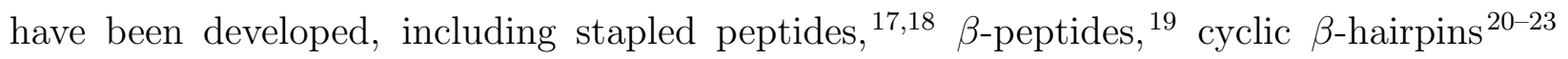
and D-peptides. ${ }^{24}$ Cyclic $\beta$-hairpins ligands, pioneered by the Robinson group, represent a particularly attractive scaffold, stabilized by cyclization, intra-strand hydrogen-bonding and D-Pro/L-Pro capping groups that mimic $\beta$-turns.

A key design principle for cyclic peptides has been the idea that cyclization and crosslinking should enhance structural preorganization, which should in turn enhance binding affinity by decreasing the unfavorable conformational entropy change upon binding. $62127 \sqrt[33]{36}$ This idea has been borne out for many different helical peptides. Helical stapled-peptides based on

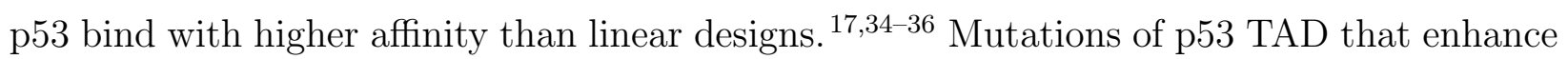
helicity also have higher affinity to MDM2 and affect signaling in cells. $\frac{37}{37}$ Markov State Model (MSM) of p53 TAD-MDM2 binding reaction, built from $831 \mu$ s of aggregate trajectory data, reveals a mechanism in which binding of p53 TAD precedes folding, which predicts increased affinity as peptide helicity increases ${ }^{38}$ An MSM of MDM2 binding for a more helical, high-affinity designed peptide PMI ${ }^{24}$ shows a mixture of induced-fit and conformational selection pathways. $\frac{39}{39}$

For cyclic $\beta$-hairpin binders of MDM2, the relationship between solution-state preorganization and binding properties is less clear. Unlike intrinsically disordered peptides, cyclic 
$\beta$-hairpins are partially folded in solution, and detailed characterization of the extent of their preorganization must come from careful comparison of biophysical measurements and simulated conformational ensembles. Recent work from Danelius et al. exploring the relationship between the rigidity of four cyclic $\beta$-hairpins and binding affinity to MDM2 offers insight into this question. $\stackrel{40}{ }$ The first of these peptides (peptide 1) was originally designed by Fasan et al. $\stackrel{23}{23}$ to mimic p53 TAD by displaying residues Phe, 6-chloro-Trp and Leu in a similar geometry (Figure 1). Peptides 2, 3 and 4 (Figure 1b) replace the D-Pro/L-Pro capping motif with a D-Pro-Gly $\beta$-turn type II' motif, substitute smaller polar residues (i.e. Glu $\rightarrow$ Ser), and explore different halogenated aromatic substitutions. Danelius et al. used solution-state NMR, along with the NAMFIS algorithm, 11 to characterize the structural heterogeneity of each peptide, as well as the affinity of the four designs, measured using SPR and fluorescence polarization assays.

Here, to further explore the relationship between preorganization, binding affinity and binding mechanism, we perform massively parallel all-atom molecular simulations of the four peptides, both in solution, and in the presence of MDM2. By collecting and analyzing a large number of $a b$ initio binding trajectories, we characterize both folding and binding mechanisms in microscopic detail.

\section{METHODS}

Molecular Simulation of Cyclic Hairpin Designs. Cyclic peptides were constructed using the AmberTools tleap program. Topologies for non-natural amino acids were built using UCSF Chimera and parameterized using the Generalized AMBER force field (GAFF), $\underline{42}$ with partial charges computed using the AM1-BCC 43 methods. The ACPYPE program $\underline{44}$ was then used to convert the topology file format for use with the GROMACS. MD simula-

tions were performed using GROMACS 4.5.4 $4^{\underline{45}}$ on the Folding@home distributed computing

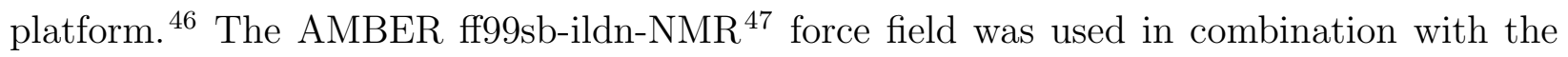


a

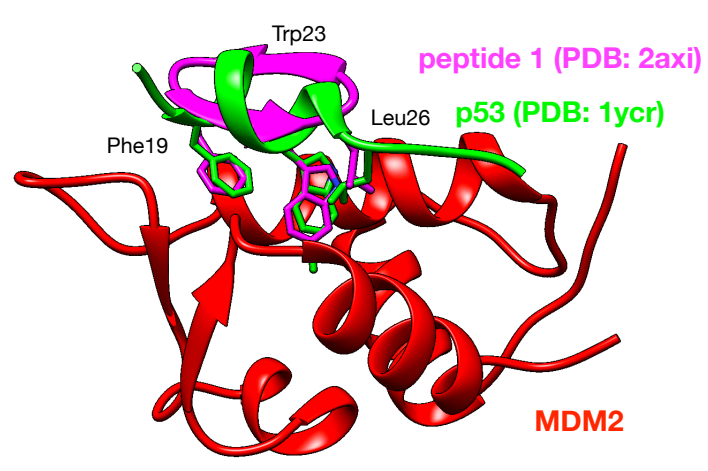

C
peptide 1 cyclo-FEULDWEF ${ }^{\mathrm{D}} \mathrm{P}^{\mathrm{L}} \mathrm{P}$
peptide 2 cyclo-FSXLDESF ${ }^{\mathrm{D}} \mathrm{PG}$
peptide 3 cyclo-FSZLDESF ${ }^{\text {DPG }}$
peptide 4 cyclo-FSULDWSF ${ }^{\mathrm{D}} \mathrm{PG}$

b

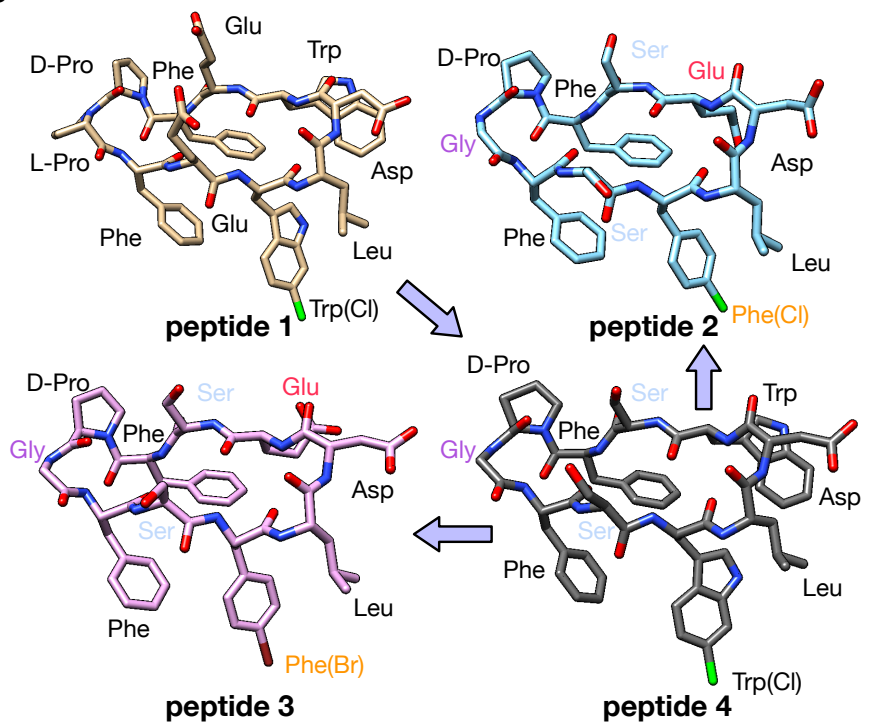

Figure 1: (a) Superposition of the crystal structure of peptide 1 bound to MDM2 (PDBid: 2axi, magenta) and the crystal structure of the p53 transactivation domain peptide (green) bound to MDM2 (red, PDBid: 1ycr). (b) Structures of the four designed cyclic $\beta$-hairpins examined in this study. Peptide 1 was originally designed by Fasan et al. ${ }^{23}$ Peptide 4 is obtained by substitution of Glu and L-Pro with Ser and Gly, respectively. Peptide 2 is obtained by further substitution of Trp and 6-chloro-(L)-Trp with Glu and 4-chloro-(L)-Phe. Peptide 3 is obtained after further replacement of 4-chloro-(L)-Phe with 4-bromo-(L)-Phe. (c) Sequences of the four peptides examined in this study. Single-letter residue codes $U=$ 6-chloro-(L)-tryptophan; $X=4$-chloro-(L)-phenylalanine; $Z=4$-bromo-(L)-phenylalanine.

TIP3P explicit solvent model. Cubic periodic boxes were filled with solvated protein and counterions $(0.1 \mathrm{M} \mathrm{NaCl})$ to neutralize the system. A full list of particle numbers and box sizes can be found in Table S1. Simulations were minimized and equilibrated at $300 \mathrm{~K}$ at a constant pressure of 1 atm for 200 ps using a Berendsen thermostat and barostat with time constant 1 ps and compressibility $4.5 \times 10^{-5} \mathrm{bar}^{-1}$. Trajectory data was generated using constant-volume molecular dynamics at $300 \mathrm{~K}$, a stochastic (Langevin) integration with a 2 fs time step, and friction constant $1 \mathrm{ps}^{-1}$.

Simulations of MDM2-bound cyclic $\beta$-hairpin peptide 1 were initiated from the crystal structure pose (PDBid: 2axi). Simulations of peptides 2, 3 and 4 were initiated from homology models built from the crystal structure template. Simulations of ab initio peptide binding to MDM2 were initiated from ten different starting configurations where the cyclic 
peptide is randomly inserted in the simulation box, and MDM2 is placed the center of the box. The distance between the center of mass of MDM2 and the peptide ligand was chosen to be at least $3.0 \mathrm{~nm}$, the distance beyond which we consider the peptide to be unbound (Figure S1). Over $3 \mathrm{~ms}$ of aggregate trajectory data was collected for analysis (Figure S2). The total simulation time and average trajectory length for each simulation can be found in Table $\$ 2$ [S3,

Umbrella simulations of cyclic hairpins. To enhance sampling of unbinding events, a series of umbrella simulations of peptide 1 were performed. A harmonic restraint with force constant $200 \mathrm{~kJ} \mathrm{~mol}^{-1} \mathrm{~nm}^{-2}$ was enforced for the distance between the $\beta$-carbon $(\mathrm{C} \beta)$ of 6-Cl-Trp on peptide 1, and the $\alpha$-carbon $(\mathrm{C} \alpha)$ of Gly58 on MDM2. Separate simulations were performed for a series of 27 different equilibrium distances ranging from $0.5 \mathrm{~nm}$ to 3.1 $\mathrm{nm}$, in increments of $0.1 \mathrm{~nm}$, resulting in $\sim 56 \mu$ s of aggregate trajectory data (Table S4).

Markov State Model (MSM) Construction. MSM approaches have been used with great success to model the conformational dynamics of protein folding and binding as a kinetic network of metastable conformational states, providing the atomistic details to help complement and interpret experimental measurements. $\frac{48 / 49}{4}$ A key advantage of MSMs is the ability to infer information about folding thermodynamics and kinetics from ensembles of short, nonequilibrium trajectories.

The MSMBuilder $3^{50}$ and PyEMMA ${ }^{51}$ software packages were used to construct MSMs from the simulation trajectory data. This involves the following steps: (1) featurizing the trajectory data, (2) projecting the time-series of features to a lower-dimensional subspace, (3) conformational clustering in this subspace to define kinetically metastable discrete states, and (4) using the observed transitions counts between discrete states to estimate $\mathrm{T}(\tau)$, the matrix of transition probabilities between discrete states at lag time $\tau$, from which all thermodynamic and kinetic properties can be inferred.

MSMs of cyclic peptides in solution. To build MSMs of peptides in solution, we used the 
pairwise distances between $\mathrm{C} \alpha$ and $\mathrm{C} \beta$ atoms as features. Dihedral angle $(\psi, \phi)$ features were also tested (resulting in poorer models, see Results section). Time-structure-based independent component analysis ${ }^{52153}$ (tICA) was used to project the distance features to a low-dimensional subspace for the purpose of conformational clustering. The outputs of tICA are the set of time-lagged independent components (tICs) that best capture a low-rank approximation of the time-lagged correlation matrix. Thus, the tICs represent the degrees of freedom along which the longest-timescale conformational dynamics occurs.

Conformational clustering was performed using the k-centers algorithm over four tICs (derived using a tICA lag time of $5 \mathrm{~ns}$ ) to identify 50 metastable states for each cyclic peptide. This number of metastable states was determined using the generalized matrix Rayleigh quotient (GMRQ) variational cross-validation method. ${ }^{54}$ A maximum-likelihood estimator (MLE) was used to estimate the MSM transition matrix from sliding-window counts. An MSM lag time of 20 ns was chosen for construction of the MSM model. Plots of implied time scales versus MSM lag time showed convergence near 20 ns, validating that dynamics was sufficiently Markovian (Figure S3). To compare differences in folding landscape between different peptides, we also construct what we call "joint MSMs" from the combined simulation data for multiple peptides. Previous work from our group showed the success of probing important differences in conformational dynamics by construction of joint MSMs with unified set of state definitions. .55 t57

MSMs of cyclic peptide binding to MDM2. To build MSMs of the peptide binding to MDM2, we chose two different featurizations for comparison. The first set of features, which we will call $\mathrm{F} 1$, consisted of pairwise distances between $\mathrm{C} \alpha$ and $\mathrm{C} \beta$ atoms in the peptide and $\mathrm{C} \alpha$ and $\mathrm{C} \beta$ atoms for selected residues in MDM2: Lys51, Leu54, Phe55, Leu57, Gly58, Gln59, Ile61, Met62, Tyr67, Gly72, Val75, Ser92, Val93, His96, Ile99, Tyr100, Ile103. (see Figure S4). The second set of features, which we will call F2, consisted of pairwise distances between $\mathrm{C} \alpha, \mathrm{C} \beta$ and side chain heavy atoms in the peptide, and $\mathrm{C} \alpha$ and $\mathrm{C} \beta$ of selected residues in MDM2 (the same as listed above). Similar featurizations were used in our previous work, 
and found to be especially useful in analyzing peptide binding dynamics at high resolution. 38

For peptide 2-4 system, all ab initio binding trajectories (started from unbound states) and selected long bound-state trajectories were used as the input data to construct MSMs. For peptide 1, all bound-state and ab initio binding trajectories (started from unbound states) were used as the input data to construct MSMs due to the large amount of data from binding simulations. The use of a combination of bound-state and binding trajectories in MSM construction has shown great success to understand binding mechanism at a higher resolution of motions. $\frac{58}{5}$ To identify trajectories in which the peptide ligand successfully binds to MDM2, the center-of-mass distance between MDM2 and the cyclic peptide was computed and compared with the reference distance $(1.2 \mathrm{~nm})$ from crystal structure (PDBid:2axi).

The GMRQ method was used to determine optimal hyperparameters for constructing MSMs (Figure S5), such as the number of metastable states (200 states for the peptide 1 system, 75 states for peptides 2, 3 and 4). A tICA lag time of 5 ns and 4 tICA components were used for tICA analysis. A lag time of 25 ns was used for all MSMs of cyclic peptide binding to MDM2.

Transition Path Theory analysis. Transition Path Theory $\underline{59}$ (TPT) analysis was performed to estimate binding pathways, fluxes, and rates using the MSMs of cyclic peptides association. Briefly, TPT uses the transition probability matrix $T^{(\tau)}$ to solve a set of selfconsistent equations to obtain committor values $q_{i}^{+}$for every state $i$. The committor value $q_{i}^{+}$is the probability that a trajectory started from state $i$ will reach a set of sink states (B) before reaching a set of source states $(\mathrm{A})$. Once the committor values are determined, the reaction rate $k_{A B}$ can be computed as $k_{A B}=\frac{F}{\tau \sum_{i} \pi_{i}\left(1-q_{i}^{+}\right)}$where $F=\sum_{i \in A} \sum_{i \notin A} \pi_{i} T_{i j}^{(\tau)} q_{i}^{+}$is the total flux and $\pi_{i}$ is the equilibrium population of state $i$. The rate $k_{A B}$ is the expected number of observed $\mathrm{A} \rightarrow \mathrm{B}$ transitions per unit time $\tau$, which can be converted to a predicted rate constant by $k_{A B}^{\prime}=k_{A B} / c$ where $c$ is the simulated molar concentration.

To calculate rates of cyclic peptide binding to MDM2, unbound states used in TPT cal- 
culations are defined based on the relative distances between the ligand and MDM2 (Figure S6). Any conformational states with an ensemble averaged distance smaller than $0.8 \mathrm{~nm}$ and larger than $3.0 \mathrm{~nm}$ are defined as bound and unbound states, respectively (Figure S7). Uncertainty estimates were calculated using a bootstrap procedure, whereby ten different MSMs were constructed by sampling the input trajectories with replacement.

Multiensemble Markov model construction. The recently developed TRAM estimator by the Noé group (transition-based reweighting analysis method) combines the features of free energy estimators like MBAR with the features of transition count-based MSM rate estimators and gives results better than either alone. $\frac{62[63}{6 n}$ order to better estimate kinetics and thermodynamic properties, a MEMM was built from the unbiased MD and umbrella simulation trajectory data. To define MEMM states, we first used tICA to project the combined simulation data of both unbiased and umbrella sampling to tICs, using as features pairwise distances between $\mathrm{C} \alpha$ and $\mathrm{C} \beta$ atoms in ligands and selected residues in MDM2 (see Figure S4). We then confirmed both unbiased and umbrella simulation share the same slow motions $\left(\mathrm{tIC}_{1}\right.$ and $\mathrm{tIC}_{2}$ ) (Figure $\mathrm{S} 8$ ). Then we used the state definitions from our MSM of peptide binding to assign state indices to each snapshot of the umbrella simulation trajectory data (based on their Euclidean distance to the cluster centers). In total, 200 microstates were used for MEMM construction. The trajectory data included both unbiased simulations starting from unbound state, and umbrella simulations starting from bound state. A lag time of 25 ns was used for TRAM estimation based on the convergence of the implied time scales (Figure S9). Uncertainty estimates were calculated using a bootstrap procedure, whereby ten different MEMMs were constructed by sampling the input trajectories from each ensemble with replacement.

Bayes Factor Analysis. A Bayes factor method was used to identify important contacts in each metastable state in MSM. Consider two sets of inter-residue contacts $\left\{C_{i j}\right\}$ and $\left\{C_{i j}\right\}^{*}$. The variables $C_{i j}$ are contact indicator variables. If a contact is present between 
residues $i$ and $j$, then $C_{i j}=1$ otherwise, $C_{i j}=0$. The Bayes factor $B F$ is defined as the ratio of probabilities that the structure is in state $k$ given the set of contacts $\left\{C_{i j}\right\}$ versus the set of contact $\left\{C_{i j}\right\}^{*}$ :

$$
B F=\frac{P\left(k \mid\left\{C_{i j}\right\}\right)}{P\left(k \mid\left\{C_{i j}\right\}^{*}\right)}
$$

Suppose the two sets of contacts differ only by a single contact, $C_{m n}$, that is formed in the first set of contacts and not formed in the second set. If we assume that each contact is statistically independent, such that $P\left(k \mid\left\{C_{i j}\right\}\right)=\prod_{i j} P\left(k \mid C_{i j}\right)$, then cancellation of terms and application of Bayes' Theorem yields:

$$
B F_{k}\left(C_{m n}\right)=\frac{P\left(k \mid c_{m n}=1\right)}{P\left(k \mid c_{m n}=0\right)}=\frac{P\left(C_{m n}=1 \mid k\right) P\left(C_{m n}=0\right)}{P\left(C_{m n}=0 \mid k\right) P\left(C_{m n}=1\right)}
$$

In practice, the Bayes factor $B F_{k}\left(C_{m n}\right)$ is estimated from the frequencies of contacts $N$ observed in the simulations, where $P\left(C_{m n}=1\right)=N\left(C_{m n}=1\right) / N_{\text {total }}$ and $P\left(C_{m n}=\right.$ $0)=1-P\left(C_{m n}=1\right), P\left(C_{m n}=1 \mid k\right)=N\left(C_{m n}=1 \mid k\right) / N_{\text {total }}$ and $P\left(C_{m n}=0 \mid k\right)=$ $\left(N_{k}-N\left(C_{m n}=1 \mid k\right)\right) / N_{\text {total }}$ where $N_{k}$ is the number of snapshots assigned to microstate $k$. A pseudo-count $N\left(C_{m n}=1 \mid k\right)=1$ is given to avoid a zero-valued denominator in the Bayes factors. Since $N_{\text {total }}$ is a very large number $(\geq 1 \mathrm{M})$ this approximation does not affect the results. In our analysis, we used the MDTraj library ${ }^{64}$ to compute contacts separated by three or more residues and define a contact formed if any pair of non-hydrogen atoms between two residues is closer than $0.4 \mathrm{~nm}$.

NAMFIS analysis. To identify the conformational families adopted in solution, we used the NMR Analysis of Molecular Flexibility In Solution (NAMFIS) algorythm, $\frac{41}{1}$ which has previously been successfully applied to describe the solution ensembles of linear peptides ${ }^{65}$ as

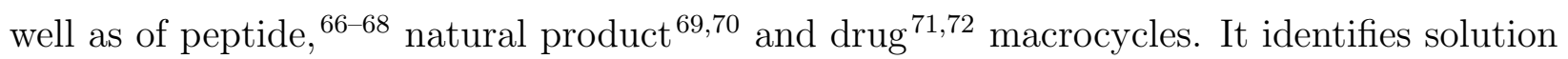
conformers and their molar fraction by deconvoluting time-averaged NMR spectroscopic data using a computationally generated ensemble as an additional input. This input ensemble has 
to cover the full conformational space available for the molecule, and hence cannot originate from a structure refinement that uses (experimental) restraints. Motivated by the poor, $<0.5$ $\mathrm{mg} / \mathrm{mL}$, aqueous solubility of the studied peptides, NOE build-up rates and scalar coupling constants were obtained by recording spectra for DMSO- $d_{6}$ solutions, similar to previous

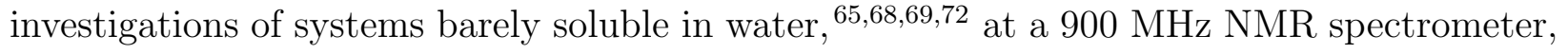
as described in detail by Danelius et al. $\underline{40}$

We used two different sets of data as theoretical input ensembles for the NAMFIS algorithm: (i) one generated from an MSM constructed using unbiased MD simulations, or (ii) one computed using restraint-free Monte Carlo conformational search, as described earlier. $\underline{40}$ The latter theoretical input ensemble was built by combination of the output conformers from two independent conformational searches using the OPLS-2005 and the AMBER* force fields, respectively, as implemented in the software Macromodel v9.1. Using only conformers within $42 \mathrm{~kJ} \mathrm{~mol}^{-1}$ from the global minimum, the ensembles were combined and redundant conformations were eliminated. The solution-state ensembles were then established by deconvolution of the experimentally obtained time-averaged interatomic distances, derived from NOE build up rates, and dihedral angles, obtained from scalar couplings, into conformational ensembles by varying the probabilities of each conformer of the priory predicted theoretical conformational pools. The conformers with non-zero probabilities were classified in terms of $\beta$-hairpin folding, with the overall folded populations being given in Table 3. We wish to emphasize that the NAMFIS algorithm is entirely driven by experimental data, and does not use any computed energies in the identification of conformers or their populations, and thereby it avoids the risks associated with the well-known method dependence of computed energies. ${ }^{[33}$ On the other hand, the reliability of its output vastly depends on the completeness of the theoretical input, which has to represent the entire theoretically available conformational space for the studied flexible compound. The quality of various input theoretical conformational ensembles may be evaluated by statistical algorithms for model comparison (see Results). 


\section{RESULTS AND DISCUSSION}

MSMs suggest a highly preorganized solution-state conformational ensemble for peptide 1. For each of the solution-state simulations of the four peptides, tICA analysis was performed as described in Methods. The projection of distance-feature trajectories to the first two tICs reveal long-lived kinetic intermediates across the folding landscape (Figure 2. Comparison of the tICA projections show that conformation sampling for peptide 1 is highly concentrated in the native-state basin, in marked contrast to peptides 2,3 and 4 , which populate a number of non-native basins.

Direct comparison of the tICA projections of the four peptides is problematic because the tICs are unique to each peptide's dynamics. To obtain a better comparison of how conformational dynamics varies across the four peptide designs, we constructed joint MSMs trained on the time-series of features for multiple peptides. In agreement with the individual tICA projections, joint tICA projections for pairs of peptides consistently show significant solution-state preorganization for peptide 1 (Figure S10 S12). Since peptide 1 has a DPro/L-Pro turn motif, while peptides 2, 3 and 4 have a D-Pro-Gly turn, the simulations suggest that replacing the highly flexible glycine with L-proline can significantly rigidify the overall cyclic peptide structure. This is consistent with known ability of D-Pro/L-Pro turn mimics to robustly induce hairpin structure. 74

To gain structural insight into the interactions that uniquely define non-native conformational basins, we computed Bayes factors to identify key inter-residue contacts as described in Methods (Figure S13,S15). For peptide 1, the most populated misfolded state is characterized by a non-native hydrogen-bond registration and interactions between Pro10 and aromatic side-chains. According to the tICA analysis, transitions between this state and the native state constitute the slowest (most time-correlated) dynamics. While this misfolded state is long-lived, few trajectories (1.13\%) sample this conformational basin. An MSM model constructed from the trajectory data predicts a population of $11.8 \%$ for all misfolded states, which is much lower than the native state $(88.2 \%$, see Table 1$)$. 

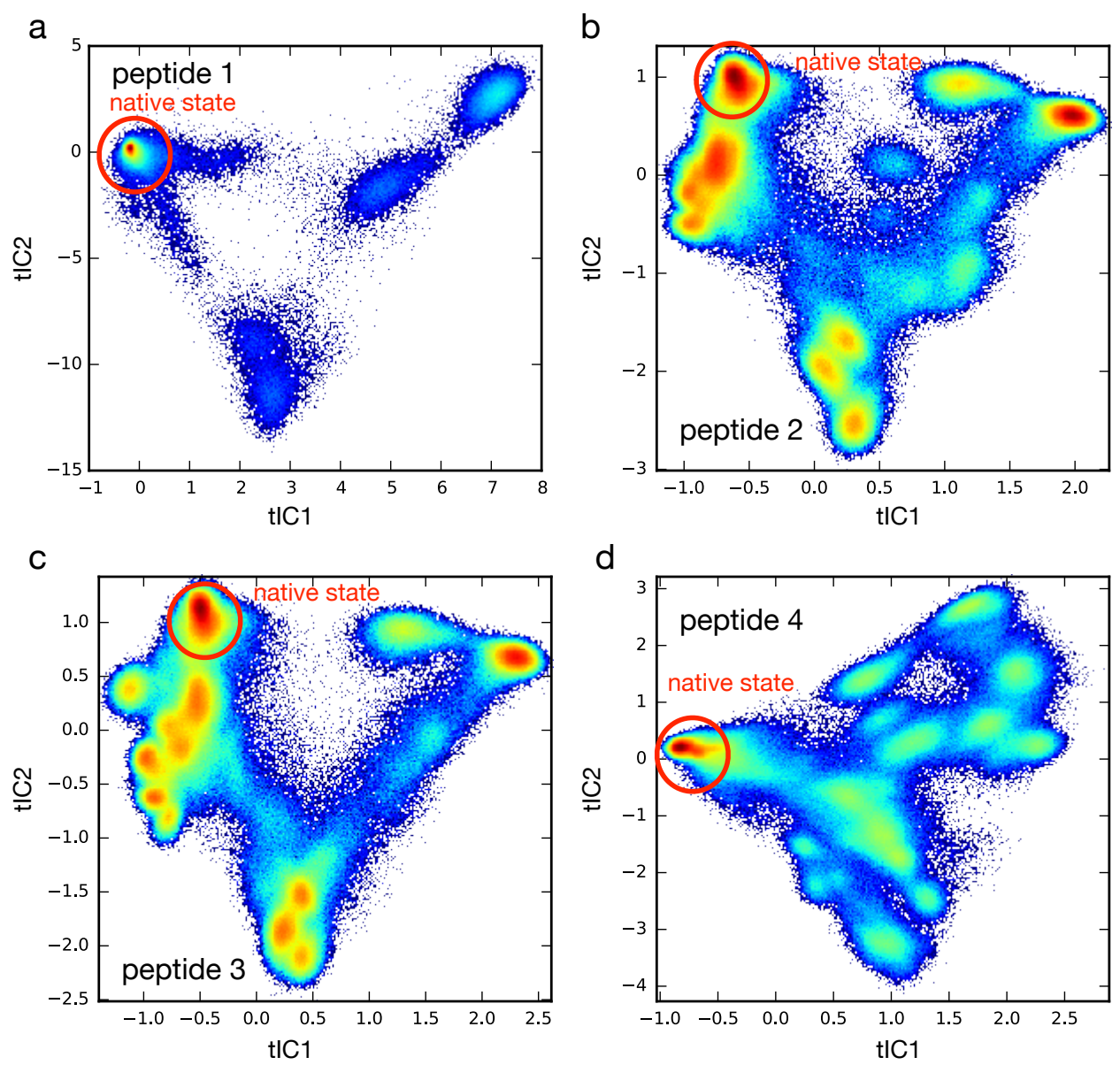

Figure 2: A heat map of trajectory data projected to the 2D tICA landscape of (a) peptide 1, (b) peptide 2, (c) peptide 3, (d) peptide 4. The native folded states are circled in red.

Peptide 2 and 3 have a similar tICA landscape (Figure 2) due to the high similarity in sequences (only the halogen atom is different). They also have the lowest folded state population compared with other peptides (Table 1). The misfolded states are mainly induced by non-native hydrogen bonding between Ser side chains and Glu/Asp side chains (Figure S14). One of the design motivations for these peptide sequences, in which Glu residues of peptide 1 are replaced by Ser, is to provide higher ligand flexibility in solution. ${ }^{40]}$ However, our results would suggest that increased flexibility introduces unexpected interactions which further lower preorganization. In addition to distance features, we also constructed MSMs using backbone dihedral angle features, to determine whether it resulted in better models of molecular kinetics. By the variational principle of conformational dynamics, $\frac{75}{75}$ the optimal 
eigenbasis to model molecular kinetics is the one that maximizes the eigenvalues of a timecorrelation matrix, which means that slower implied timescales indicate a better model (assuming finite-sampling/over-training errors are negligible). We find that dihedral angle features lead to faster timescales than distance features (Figure S16), and therefore reject this approach in favor of pairwise distance featurization.

\section{Binding affinities of cyclic peptides are highly correlated with solution-state sta-}

bility. While tICA analysis provides a qualitative analysis of the relative stability of peptides in solution, we can quantitatively assess the preorganization of the peptides using two metrics: (1) the native state population predicted by the MSMs, and (2) the $\beta$-strand content as computed by the Dictionary of Protein Secondary Structure (DSSP) algorithm. Both metrics provide identical rankings of the preorganization of the four peptides (Table 1 ). Comparison of predicted native state population with experimental binding affinities $\underline{40}$ reveals a remarkable correlation $\left(R^{2}=0.950\right)$ between preorganization and experimental binding affinity (Figure 3a). This result suggests an expanded view in which not only cyclization can stabilize peptides to enhance their affinity, $\underline{17 / 76 / 77}$ but sequence-based modulation of their intrinsic flexibility and folding properties.

To further explore the relationship between solution-state preorganization and binding affinity, we examined the applicability of two simple models. First, we consider a conformational selection model in which the peptide must fold completely before binding to MDM2. In this case, the predicted dissociation constant is $K_{D}=K_{D}^{*}\left(1+K_{u}\right)$, where $K_{D}^{*}$ is the dissociation constant for a folded (i.e. completely preorganized) peptide, and $K_{u}$ is the unfolding equilibrium constant (see SI for derivation). Using least-squares fitting of $\ln K_{D}^{*}\left(1+K_{u}\right)$ to the experimental $\ln K_{D}$, we found that $K_{D}^{*}=0.519 \mu \mathrm{M}$ best matched the simulation results, with reasonable correlation $\left(R^{2}=0.793\right)$ between theory and experiment (Figure $3 \mathrm{~b}$ ).

Going beyond this simple two-state folding model, we next considered a model in which the free energy of binding $\Delta G_{b i n d}$ is related to the change in the peptide's conformational 
entropy upon binding, $\Delta S_{b i n d}$. To see if this was the case, we used a featurization that only included pairwise distances of atoms in the ligand to construct joint MSMs from simulated trajectories of 1) the ligand in solution, 2) the ligand bound to MDM2, and 3) ab initio binding simulations. As expected, when bound with MDM2 (no unbinding events were observed), a very restricted set of conformational states are visited by peptide 1-4, and more conformational heterogeneity is observed when the peptide if unbound (Figure 4 , S17, S19). The value of $T \Delta S_{\text {bind }}$ was estimated as $T\left(S_{b}-S_{u}\right)$ where $S_{u}$ and $S_{b}$ are peptide conformational entropies in the unbound and bound states, respectively, calculated from MSMs built from trajectory data of the ligand in solution, and ligand bound to MDM2, respectively. Entropies were estimated using the formula $S=-R \Sigma_{i} p_{i} \ln p_{i}$ where $p_{i}$ are the metastable state populations of the MSM.

If we assume that the different binding free energies of the four peptides are due entirely

to differences in $\Delta S_{b i n d}$, we would expect that the experimental values of $\ln K_{D}$ vs. $T \Delta S_{b i n d}$ would obey a linear relationship, with a slope of $-1 / R T$. Indeed, a scatter plot shows these values to be highly correlated $\left(R^{2}=0.962\right)$, with nearly exactly the expected slope (Figure 3c). This result suggests that solution-state preorganization completely determines the binding affinity of the four peptides. Moreover, it suggests that atomically-detailed simulation models may be essential for predicting this kind of information.

We note that such strong correlation with solution-state entropy is likely due in part to the high similarly of the four peptides' residues that form an interface with MDM2, and might not be observed for sequence variants which more directly perturb the binding interface. Other studies have also pointed out counterexamples where enhanced preorganization results in weaker binding, suggesting that greater flexibility may be necessary for some designed ligands. $\stackrel{78179}{ }$

Comparison of simulated ensembles with structural NMR measurements. To validate the accuracy of our simulated ensembles, we compared simulated interproton dis- 
Table 1: Stability predicted from simulations vs. binding affinities measured from experiments.

\begin{tabular}{c|l|l|l} 
Peptide & $\begin{array}{l}\beta \text {-strand con- } \\
\text { tent in solution } \\
(\mathrm{DSSP})(\%)\end{array}$ & $\begin{array}{l}\text { folded state popu- } \\
\text { lation in solution } \\
(\mathrm{MSMs})(\%)\end{array}$ & $\begin{array}{l}\text { Binding affinities } \\
K_{D}(\mu \mathrm{M})\end{array}$ \\
\hline $\mathbf{1}$ & 33.55 & 88.19 & $0.127 \pm 0.001$ \\
$\mathbf{2}$ & 0.74 & 11.81 & $7.00 \pm 0.10$ \\
$\mathbf{3}$ & 0.56 & 11.48 & $5.73 \pm 0.09$ \\
$\mathbf{4}$ & 19.91 & 47.77 & $2.50 \pm 0.02$
\end{tabular}
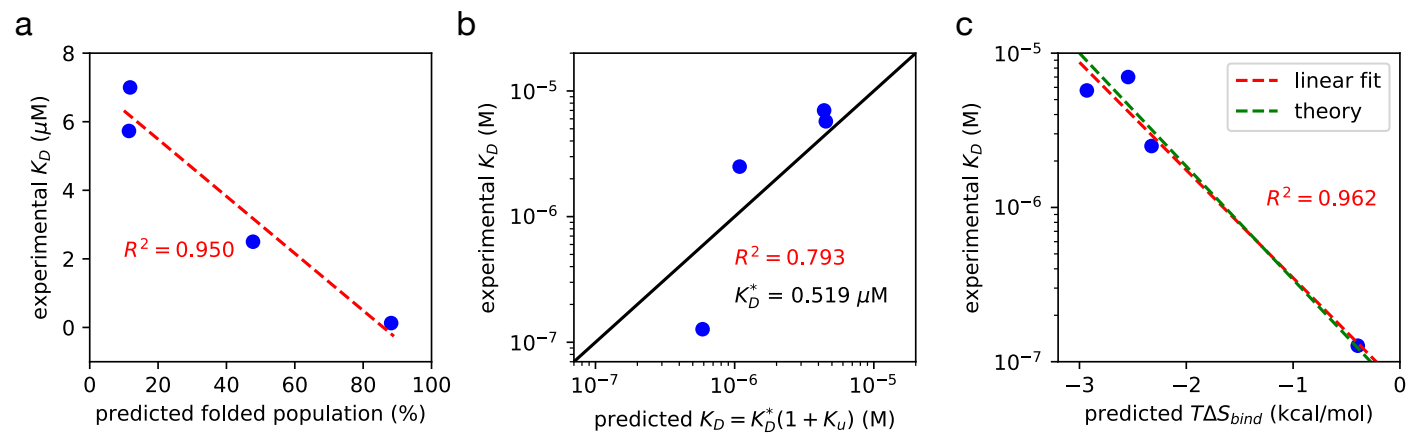

Figure 3: (a) Experimentally measured dissociation constants versus folded state population predicted by MSMs. (b) Experimentally measured dissociation constants versus predictions from a three-state conformational selection model. (c) Experimentally measured dissociation constants versus $-T \Delta S_{\text {bind }}$.

tances to NOE measurements published by Danelius et al. $\stackrel{40}{ }$ Simulated ensemble-averaged NOE distances $d_{i}$ were computed as $d_{i}=\sum_{j} \pi_{j}\left\langle r_{i}^{-6}\right\rangle_{j}^{-1 / 6}$ where $\pi_{j}$ is the predicted equilibrium population of MSM state $j, r_{i}$ is distance for proton pair $i$, and the bracketed term represents the ensemble average over conformations belonging to state $j$.

A comparison of experimental and predicted NOE distances (Figure 5) shows rms differences to be $0.16,0.26,0.27$, and $0.13 \mathrm{~nm}$, for peptides $1-4$, respectively. These differences are comparable to the rms differences $(0.12,0.06,0.08$ and $0.04 \mathrm{~nm}$ for peptides $1-4$, respectively) of the back-calculated solution structures solved by Danelius et al. Moreover, we constructed a representative collection of proton pairs that do not exhibit experimental NOEs by random selection from the set of NOE protons. We excluded those adjacent residues 

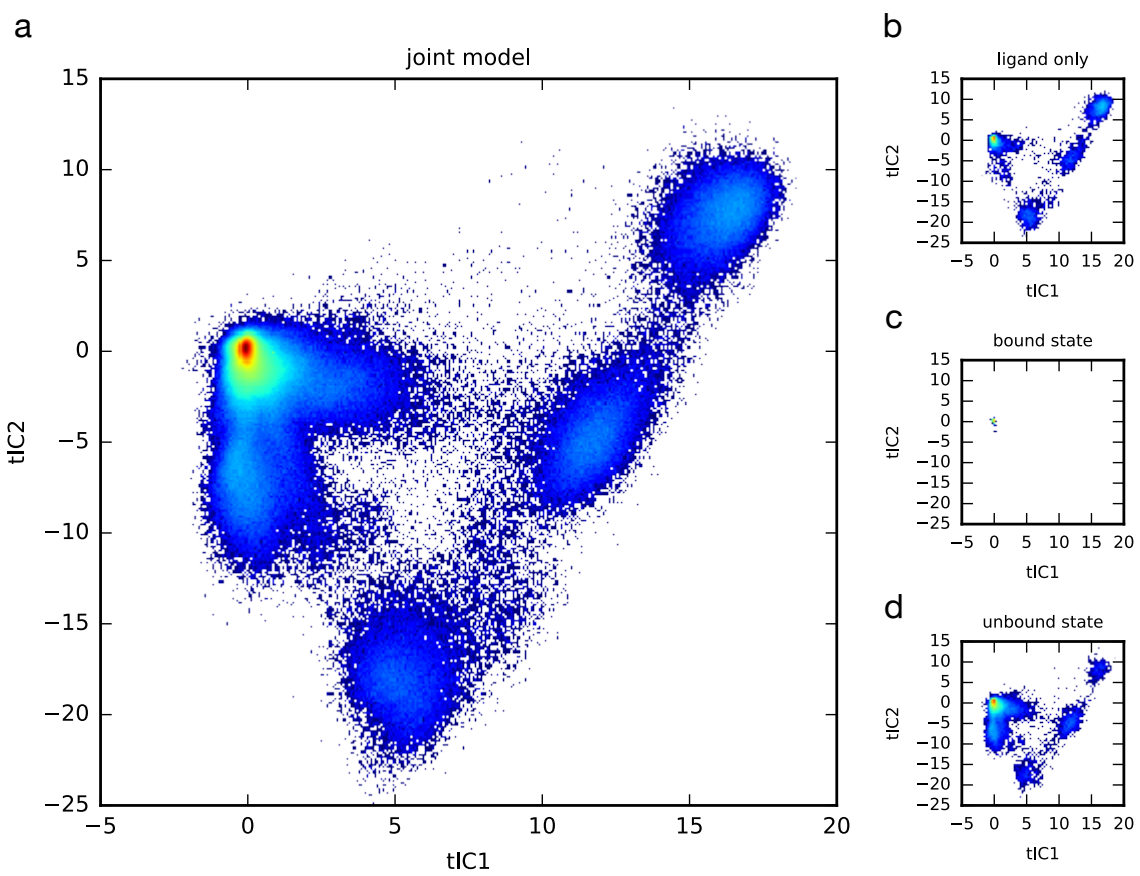

Figure 4: (a) Combined trajectory data of peptide 1 projected to the 2D tICA landscape. (bd) Transformation of simulation trajectory data using tICA model generated from combined trajectory data for ligand only, bound state and unbound state.

and found their predicted distances nearly always exceed $\sim 0.75 \mathrm{~nm}$. Overall, these results suggest the simulated ensembles agree well with with the experimental NMR spectroscopy.

A confusing discrepancy between our work and that of Danelius et al. was the apparent disagreement in the extent of folded structure reported for peptides 1-4. In Danelius et al., peptides 2 and 3 are reported as having the greatest extent of $\beta$-hairpin structure, while our simulations predict that peptide 1 has the greatest extent of folded structure. A deeper analysis, however, shows these disagreements are superficial. In Danelius et al., the NAMFIS algorithm ${ }^{41}$ was used to determine an ensemble of structures compatible with NOE constraints, and their relative populations. These populations were in turn used to characterize the extent of $\beta$-hairpin population in solution, according to a metric where any structures with at least three hydrogen bonds (defined as hydrogen bond donor and acceptor distance within $0.3 \mathrm{~nm}$ ) are considered as folded to a hairpin. When we input our MSM states and the ensemble averaged NOE distances to NAMFIS, we achieve excellent agreement 

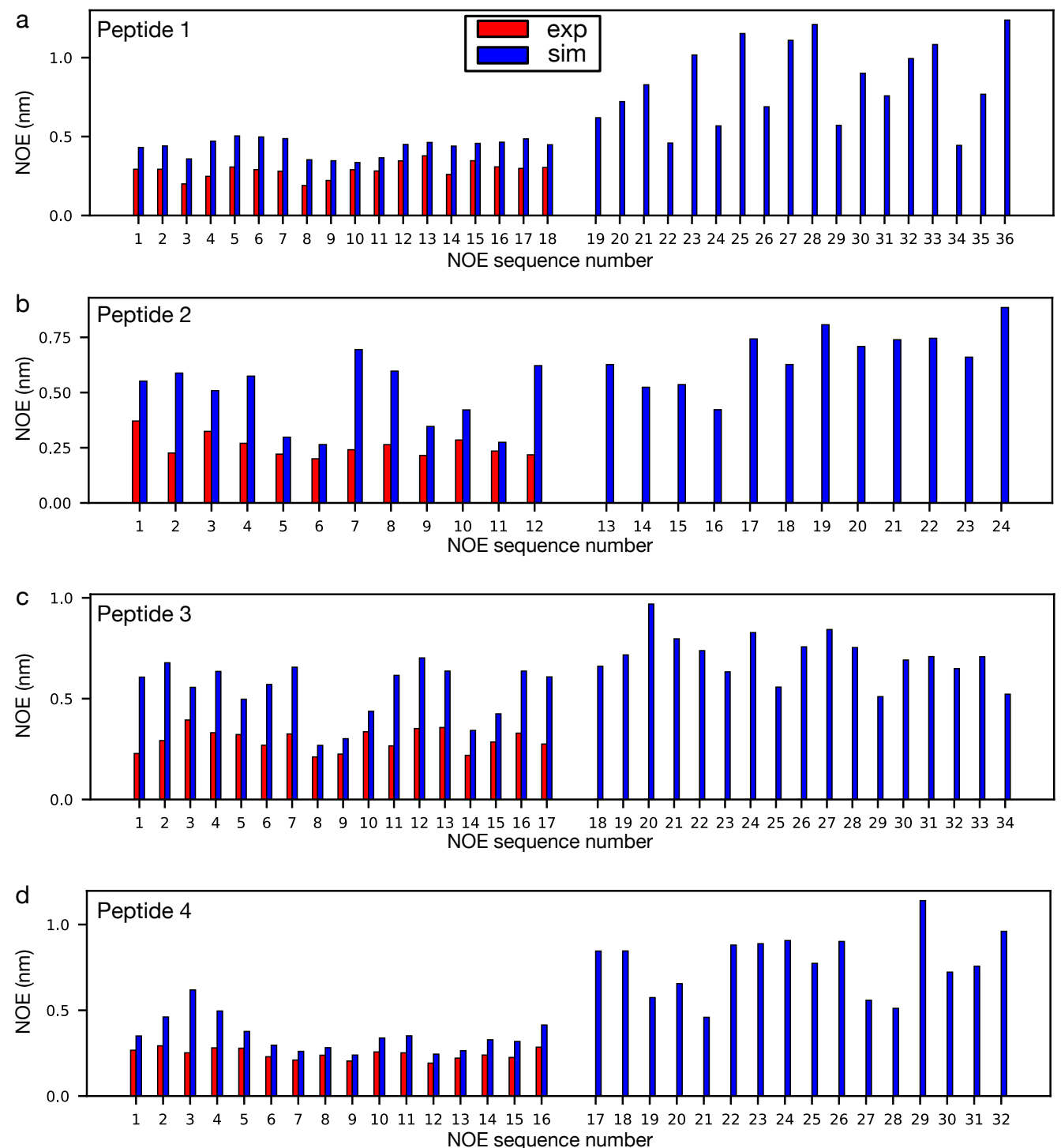

Figure 5: Comparison of experimental and predicted NOE distances for peptides 1-4 (panels a-d). In each panel, the red bars show experimental measured NOEs and the blue bars show population reweighted predicted NOEs. The left side of each plot shows predicted interproton distances for which NOEs were experimentally observed. The right side of each plot shows predicted distances for proton pairs that do not show experimental NOE. (The full list proton pairs are described in Table $\mathrm{S} 5 \mathrm{~S} \mathrm{~S} 8$.)

with the results reported by Danelius et al. (Table 3).

Further comparison of the two input ensembles was performed using Akaike Information

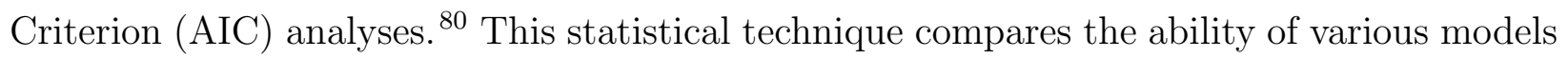
to describe reality, that is the fit of the models to experimental obtained observables. Model 
selection is important, as an under-fitted model may not capture the true conformational variability of a flexible molecule, whereas an over-fitted one lacks generality. The summary of the AIC analyses of the input models (i) and (ii) for the four peptides are given in Table 2 .

Table 2: Summary of AIC results for the theoretical models used as input for the estimation of the $\beta$-hairpin populations of peptides 1-4.

\begin{tabular}{lccccccc}
\hline Model & SSD & AIC & AICc & $\Delta$ AICc & $\mathrm{w}_{i}$ & $\mathrm{ER}_{i}$ & $\mathrm{LER}_{i}$ \\
\hline Monte Carlo & 576.5 & 594.5 & 595.9 & 0.0 & 1.0 & 1.0 & 0 \\
MD sim. & 815.1 & 851.1 & 873.1 & 277.2 & 0.0 & $1.6 \mathrm{e} 60$ & 60.2 \\
\hline & & & \multicolumn{5}{c}{ Peptide $\mathbf{2}$} \\
Monte Carlo & 150.9 & 164.9 & 166.1 & 0.0 & 1.0 & 1.0 & 0 \\
MD sim. & 459.8 & 471.8 & 473.8 & 307.7 & 0.0 & $6.6 \mathrm{e} 51$ & 66.8 \\
\hline & & & \multicolumn{5}{c}{ Peptide 3} \\
Monte Carlo & 227.7 & 241.7 & 242.9 & 0.0 & 1.0 & 1.0 & 0.0 \\
MD sim. & 509.5 & 515.5 & 516.0 & 273.1 & 0.0 & $2.0 \mathrm{e} 59$ & 59.3 \\
\hline & & & \multicolumn{7}{c}{ Peptide 4} \\
Monte Carlo & 147.5 & 165.5 & 167.4 & 0.0 & 1.0 & 1.0 & 0.0 \\
MD sim. & 357.9 & 367.9 & 369.2 & 201.8 & 0.0 & $6.8 \mathrm{e} 43$ & 43.8 \\
\hline
\end{tabular}

SSD is the sum of square differences between the measured and modelled distances, as defined by Snyder et al., 81 along with the error estimates given in the same article (NOE distance $<2.5 \AA: \pm 0.1 \AA$ error, NOE distance $2.5-3.0 \AA: \pm 0.2 ; 3.0-3.5 \AA: \pm 0.3 ;$ NOE distance $3.5-6.0 \AA: \pm 0.4$ ). AIC is the Akaike information criterion, AICc is the small sample size-corrected AIC that has a compensation for over-fitting for systems with small sample size, $\Delta \mathrm{AICc}$ is the difference in AIC of an individual model as compared to the best model, $\mathrm{w}_{i}$ is the Akaike weight of evidence in favor of a model being the actual best model for the given data, $\mathrm{ER}_{i}$ is the evidence ratio describing the relative likelihood of a pair of models, and $\mathrm{LER}_{i}$ is the logarithmic evidence ratio. The difference in $\mathrm{LER}_{i}$ expresses the degree by which the best proposed model is better than all other anticipated models. Accordingly, the model best fitting with reality has $\mathrm{LER}_{i} 0$, and an $\mathrm{LER}_{i}$ difference of 0 - 0.5 is interpreted as "weak", 0.5 - 1 as "substantial", $1-2$ as "strong", and > 2 as "decisive" evidence.

The AIC analyses (Table 2) suggests that there is a decisive evidence that the Monte 
Carlo-derived input ensemble is the more robust model. This means that the NAMFIS output derived using the Monte Carlo conformational search better fits to the experimental data, as this input ensemble contains all, or at least more conformers that are present in the real-life solution. There several possible explanations for this result, perhaps the most important being that the MD simulations (and subsequent MSMs) were performed in aqueous solution, whereas the NMR data was obtained for DMSO-solutions (made necessary by the low aqueous solubility of the studied peptides). Another explanation is provided by the nature of the conformational search methods. A Monte Carlo conformational search provides a highly diverse set of conformers, but an unreliable energetic ranking (not used in the NAMFIS analyses), whereas molecular dynamics is capable of reliably identifying the dominant solution conformers. Thus, MD methods may have difficulty discovering lesspopulated conformers, which in turn may complicate the ensemble fitting to experimental data. Finding the relevant conformations of macrocycles in solution has previously been shown to pose a significant challenge. $\frac{82}{8}$

That said, it is likely that the NAMFIS analysis does not agree with the MSM predictions of folded state populations (Table 1) because of the major differences in the information each algorithm uses to arrive at conformational population estimates. MSMs rely heavily on the simulation model, estimating conformational populations based on transitions observed between metastable stables, with the only restraints being detailed balance. NAMFIS, as mentioned above, relies much more on restraints derived from experimental observables, estimating conformational populations from a constraint-based analysis of states with viable structures. A more thorough analysis comparing the performance of MSMs (which can reconciled with experimental restraints using the BICePs algorithm $\frac{[83}{85}$ ) and NAMFIS remains an interesting topic for future research.

Binding mechanisms of designed cyclic MDM2 ligands. A powerful advantage of MSMs is the ability to obtain information about both kinetics and thermodynamics. Towards 
Table 3: NAMFIS predicted folded state population.

\begin{tabular}{c|l|l} 
Peptide & $\begin{array}{l}\text { Previous published NAM- } \\
\text { FIS analysis }\end{array}$ & $\begin{array}{l}\text { NAMFIS predicted folded } \\
\text { state population from } \\
\text { MSMs using a H-bonding } \\
\text { metric }(\%)\end{array}$ \\
\hline $\mathbf{1}$ & 24 & 20 \\
$\mathbf{2}$ & 61 & 63 \\
$\mathbf{3}$ & 61 & 56 \\
$\mathbf{4}$ & 39 & 57
\end{tabular}

this end, we performed MD simulations of MDM2 with cyclic peptide ligands initiated from bound and unbound states, which generated hundreds of ab initio binding events. The resulting trajectory data was combined and used to construct MSMs of the binding reaction, as described in Methods. Roughly equal numbers of binding events were observed for all ten starting positions of the ligand, which suggests that the simulated binding dynamics is not biased by the initial configuration (Figure S21).

For peptides 2, 3 and 4, we find that the tICA landscapes (i.e. the projection of the trajectory data to the first two tICs) and the resulting MSMs are similar. As an example, we present the tICA landscape (constructed using feature set F1, see Methods) for peptide 4 (Figure 6). Results for peptides 2 and 3 are shown in Figure $\mathrm{S} 22$. For these peptides, the slowest motion (along $\mathrm{tIC}_{1}$ ) corresponds to peptide binding, while the next-slowest motion (along $\mathrm{tIC}_{2}$ ) corresponds to peptide folding. The tICA landscape shows the vast majority of binding pathways must first visit intermediates where the peptide is folded, but not yet bound to MDM2 in the correct binding pose. Thus, we conclude that designed cyclic peptides 2, 3 and 4 bind via a conformational selection mechanism.

In contrast to peptides $2-4$, peptide 1 shows very little unfolding, resulting in a unique tICA landscape in which most metastable states have folded peptide conformations similar to the crystal pose (Figure 7). This finding is consistent with our simulations of cyclic peptides in solution, which show that peptide 1 has the highest extent of preorganization, 


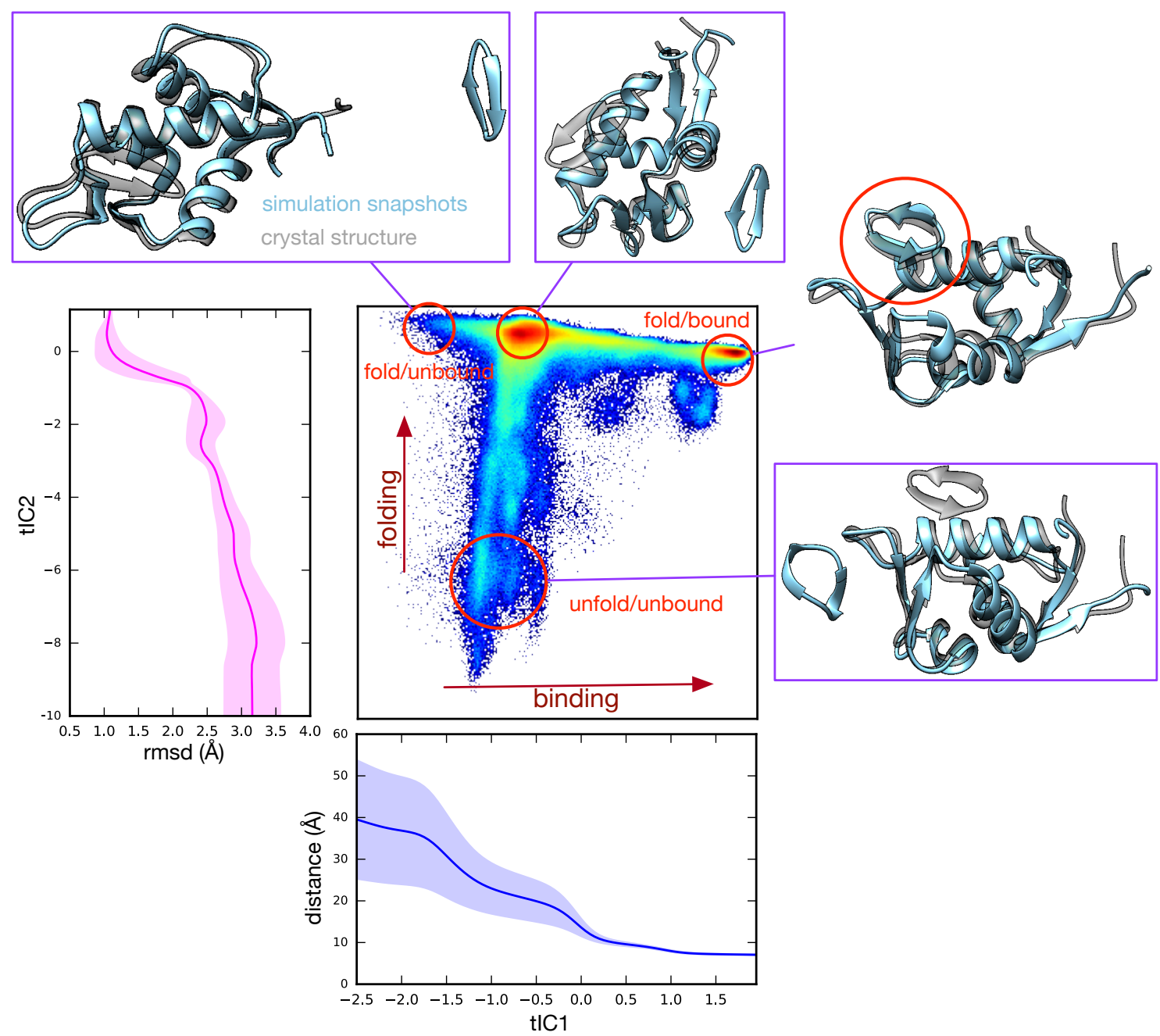

Figure 6: The slowest dynamics (along $\mathrm{tIC}_{1}$ ) corresponds to the binding process whereas the next-slowest dynamics corresponds to the ligand folding process. A conformational selection binding mechanism is revealed from the tICA landscape. Averaged distances between selected residues on MDM2 and the ligand (see Figure S5) is shown in blue and rmsd change along $\mathrm{tIC}_{2}$ is shown in magenta.

and a more stable folded state than peptides $2-4$. For this system, we found that feature set F2 (which includes heavy atoms of peptide side chains) produced superior models. The use of the F2 feature set resulted in slower implied timescales than F1, suggesting an MSM less prone to lumping errors, and better able to capture the relevant dynamics (Figure S23a-b). Moreover, the F2 featurization was able to discern binding to the correct pose on the 2D tICA landscape (Figure S23-f).

Similar to the tICA landscapes of peptides 2, 3 and 4, dynamical motion along the largest 
tICA component $\left(\mathrm{tIC}_{1}\right)$ corresponds to binding of the cyclic peptide to the crystallographic pose (Figure $7 \mathrm{~d}$ ). Motion along the next-slowest component $\left(\mathrm{tIC}_{2}\right)$, however, represents binding of the peptide to two major off-pathway traps, in poses that differ from the crystal structure (Figure $7 \mathrm{~b}, \mathrm{~h}$ ). The center of the tICA landscape corresponds to the unbound state; therefore, most trajectories that visit these "trap" states must first unbind to correctly reach to the crystallographic bound-state pose.

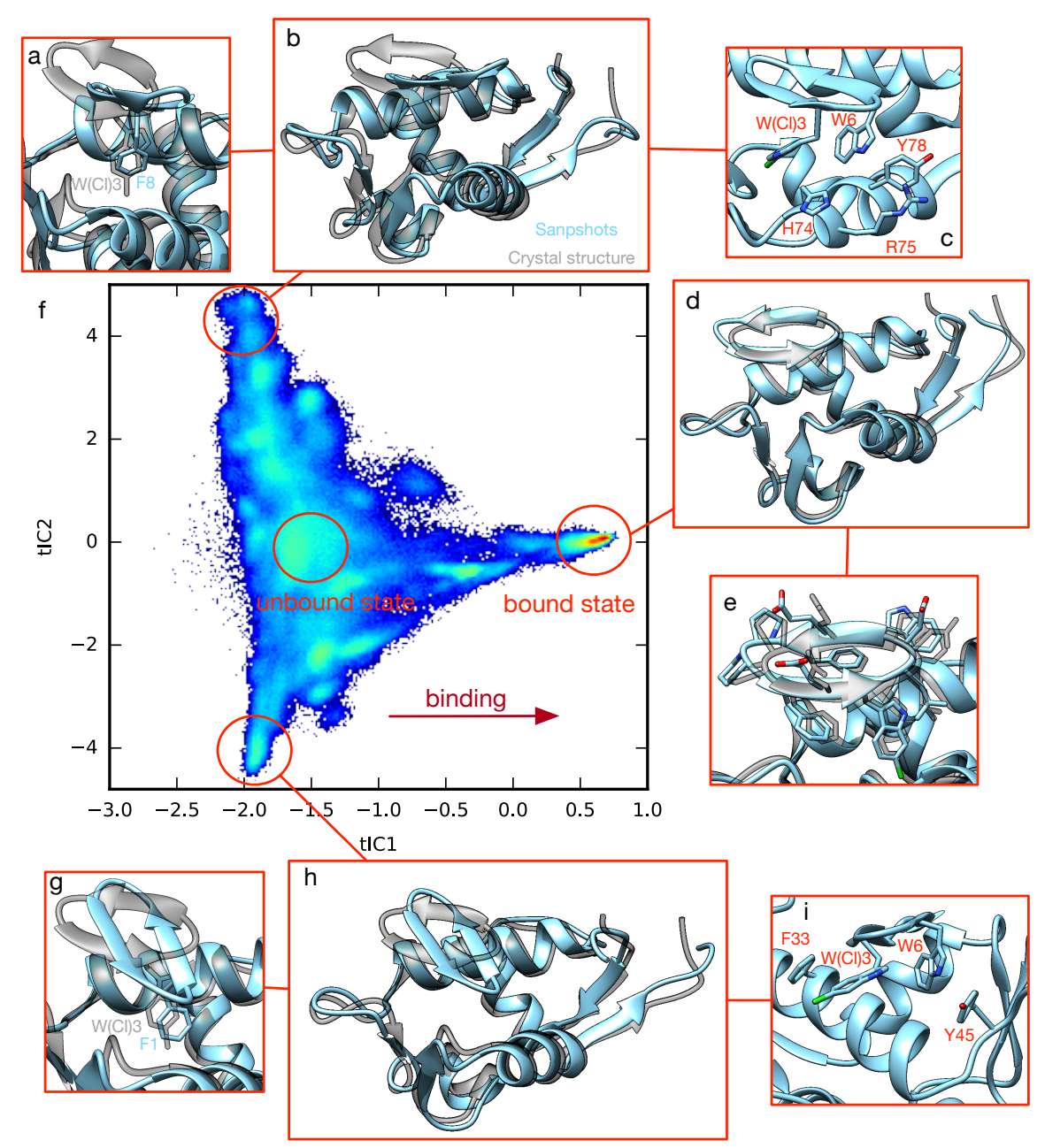

Figure 7: The slowest dynamics (along $\mathrm{tIC}_{1}$ ) corresponds to the binding process whereas the next-slowest dynamics corresponds to the ligand binding with different poses other than the crystal structure. Snapshot from simulation trajectories with key interactions observed are shown for selected metastable states.

Further inspection of the two trap states reveal near-bound poses in which hydrophobic residues designed to mimic the "hot spot" residues of Phe19, Trp23, and Leu26 of p53- 
TAD are mismatched with their preferred pocket in the MDM2 receptor. In peptide 1, 6-chloro-Trp (residue 3) is designed to mimic Trp23 in p53-TAD. In the two near-bound poses, we find instead that Phe8 (Figure $7 \mathrm{a}$ ) and Phe1 (Figure 7 $\mathrm{g}$ ) occupy the pocket in MDM2 where Trp23 normally residues. These binding poses are further stabilized by $\pi$ stacking interactions between His74-Trp $(\mathrm{Cl}) 3$, Trp6-Tyr78 (Figure 7c) and Phe33-Trp(Cl)3, Trp6-Tyr45 (Figure 7i).

The significance of the trap states is intriguing to consider. Because the folding of peptide 1 is so robust, such trap states likely represent long-lived local free energy minima which may compete with the crystallographic bound state. Discovering "trap" binding modes in computational peptide design efforts may be useful in either penalizing off-pathway poses, or in discover alternative motifs that could be further optimized.

Predicted binding rates of cyclic peptide 1 to MDM2. To estimate the kinetics of cyclic peptides binding to MDM2, we computed mean-first-passage times (MFPTs) using TPT analysis based on constructed MSMs (see Methods). We present results only for peptide 1, for which experimental measurements performed using surface plasmon resonance (SPR) have been published by Danelius et al. 10 The predicted binding rate, $k_{\text {on }}$, of peptide 1 agrees with the experimental measurement within an order of magnitude (Table 3). The estimated unbinding rate, $k_{\text {off }}$, and the bound-state population, $\pi_{\text {bound }}$, however, are poorly underestimated. Similar results were found for an MSM model of p53 TAD binding to MDM2, $\sqrt[38]{ }$ and the reason for it is well-known: MSM estimators assume that input trajectory data is sampled at equilibrium, which is impractical for binding simulations of this kind. To realize this, consider a scenario in which a peptide has dissociation constant of $K_{D} \sim 100$ nM. At our simulated concentration, we should expect only 3 of 100,000 samples to be in the unbound state. For MSM estimators that enforce detailed balance (as used in this study), input data that includes more than this fraction of unbound-state samples would tend to underestimate $k_{\mathrm{on}}$ and overestimate $k_{\text {off }}$. For MSM estimators that do not enforce 
detailed balance (such as a row-normalized counts estimator), finite-sampling error will affect estimates similarly.

To improve these estimates, we were interested in constructing multi-ensemble Markov models from umbrella simulations of peptide unbinding (see Methods) using an estimator called TRAM (Transition-based Reweighting Analysis Method) ${ }^{62}$ which uses samples from multiple thermodynamic ensembles to estimate both free energies of metastable states and the transition rates between them. The approach has been used to much more accurately estimate slow dissociation rates of ligands, including for high-affinity designed peptide ligands of MDM2. $\underline{63}$

We find that rate estimation using TRAM greatly improves the accuracy of both $k_{\text {off }}$ and $\pi_{\text {bound }}$ when compared to the conventional MSM (Table 3). Specifically, the TRAM estimation of $\pi_{\text {bound }}(\sim 96 \%)$ agrees better with the experimental value $(99.997 \%)$. The TRAM estimate of $k_{\text {on }}$ is similarly improved over the conventional MSM estimate (smaller by a factor of 20), and is within two orders of magnitude from the experimental value. While these predictions are far from perfect, the improvements are remarkable given that the total amount of biased trajectory data (from umbrella simulations, see Methods) used in the calculation is only $\sim 4 \%$ of the unbiased data.

These results show the utility of multi-ensemble Markov models in accurately estimating kinetics and thermodynamic of peptide binding, which bodes well for future studies using greater amounts of biased trajectory data. Accurate prediction of $k_{\text {off }}$ is highly desirable, as the dissociation rate is recognized as an important quantity in drug efficacy and safety in drug discovery. $\underline{\sqrt[86]{88}}$ Much pioneering work has recently been done to develop improved methods for predicting dissociation rates of protein-ligand interactions, $, 99,92$ and we are eager to contribute efforts towards this goal. 
Table 4: Estimated kinetics and thermodynamics properties using different estimators. Uncertainties are derived from a bootstrap procedure.

\begin{tabular}{c|c|c|c}
\hline Estimator & Estimated $\pi_{\text {bound }}(\%)$ & Estimated $k_{\text {on }}\left(\mathrm{s}^{-1} \mathrm{M}^{-1}\right)$ & Estimated $k_{\text {off }}\left(\mathrm{s}^{-1}\right)$ \\
\hline MSM & $51.74 \pm 9.48$ & $7.34( \pm 2.13) \times 10^{6}$ & $795.07 \pm 220.38$ \\
TRAM & $96.12 \pm 4.25$ & $7.72( \pm 5.10) \times 10^{6}$ & $40.11 \pm 39.74$ \\
Experiment & $99.997^{*}$ & $8.30( \pm 0.02) \times 10^{5}$ & $1.074( \pm 0.001) \times 10^{-1}$ \\
\hline \multicolumn{2}{r}{$*$}
\end{tabular}

${ }^{*}$ derived from experimental measured $K_{D}=129.3 \pm 0.3 \mathrm{nM}$ (see SI).

\section{CONCLUSION}

We have used molecular simulation approaches to understand the relationship between solution-state preorganization, binding mechanism, and binding affinity for four designed cyclic peptide ligands of MDM2. The microscopic view of conformational dynamics provided by Markov models reveals good agreement with previously published NMR studies, and a strong correlation between the loss of conformational entropy upon binding, and the experimental binding free energy. The best model explaining this correlation is one where solution-state preorganization completely determines the binding affinity of the four peptides, suggesting that simulation-based prediction of the solution-state properties of cyclic peptide binders may be key to discovering peptides with enhanced binding affinity.

Markov state models of the binding reaction for the four cyclic peptides show a conformational selection mechanism of binding. Even for the most preorganized design, peptide 1, non-native "trap" states are present, which could be designable features in future computational design studies. MSMs give reasonable agreement with experiment binding rates, which can be improved using the TRAM estimator alongside additional umbrella sampling simulations.

Notes All input files, setup and subset of simulated trajectory data (no water or ions) are available at: https://doi.org/10.5281/zenodo.3777274 (solution-state simulation trajectories of all peptides, https://doi.org/10.5281/zenodo.3780463 (bound-state trajectory 
data peptides 1, 2 and 3), https://doi .org/10.5281/zenodo.3782629 (bound-state trajectory data for peptide 4), https://doi.org/10.5281/zenodo.3780467 (ligand-binding trajectories of all peptides), https://doi.org/10.5281/zenodo.3814057 (umbrella-sampling trajectory data).

\section{ACKNOWLEDGEMENTS}

This research was supported in part by the National Institutes of Health through grant 1S10OD020095-01. Y.G and S.Z was supported by National Institutes of Health grant 1R01GM123296-01. This research includes calculations carried out on Temple University's HPC resources and thus was supported in part by the National Science Foundation through major research instrumentation grant number 1625061 and by the US Army Research Laboratory under contract number W911NF-16-2-0189. We would like to thank the participants of Folding@home, without whom this work would not be possible. The author also appreciates the insightful discussions with conference attendees during the poster session of the Biophysical Society Annual Meeting in 2019.

\section{References}

(1) Malde, A. K.; Hill, T. A.; Iyer, A.; Fairlie, D. P. Crystal Structures of Protein-Bound Cyclic Peptides. Chemical Reviews 2019, 119, 9861-9914.

(2) Nielsen, D. S.; Shepherd, N. E.; Xu, W.; Lucke, A. J.; Stoermer, M. J.; Fairlie, D. P. Orally Absorbed Cyclic Peptides. Chemical Reviews 2017, 117, 8094-8128.

(3) Dougherty, P. G.; Sahni, A.; Pei, D. Understanding Cell Penetration of Cyclic Peptides. Chemical Reviews 2019, 119, 10241-10287.

(4) Qian, Z.; Dougherty, P. G.; Pei, D. ScienceDirect Targeting intracellular protein-protein 
interactions with cell-permeable cyclic peptides. Current Opinion in Chemical Biology 2017, 38, 80-86.

(5) Wakefield, A. E.; Wuest, W. M.; Voelz, V. A. Molecular simulation of conformational pre-organization in cyclic RGD peptides. Journal of Chemical Information and Modeling 2015, 55, 806-813.

(6) Demmer, O.; Frank, A. O.; Hagn, F.; Schottelius, M.; Marinelli, L.; Cosconati, S.; Brack-Werner, R.; Kremb, S.; Wester, H.-J.; Kessler, H. A Conformationally Frozen Peptoid Boosts CXCR4 Affinity and Anti-HIV Activity. Angewandte Chemie International Edition 2012, 51, 8110-8113.

(7) Joerger, A. C.; Fersht, A. R. The tumor suppressor p53: from structures to drug discovery. Cold Spring Harbor Perspectives in Biology 2010, 2, a000919-a000919.

(8) Kubbutat, M. H.; Jones, S. N.; Vousden, K. H. Regulation of p53 stability by Mdm2. Nature 1997, 387, 299-303.

(9) Haupt, Y.; Maya, R.; Kazaz, A.; Oren, M. Mdm2 promotes the rapid degradation of p53. Nature 1997, 387, 296-299.

(10) Momand, J.; Zambetti, G. P.; Olson, D. C.; George, D.; Levine, A. J. The mdm-2 oncogene product forms a complex with the p53 protein and inhibits p53-mediated transactivation. Cell 1992, 69, 1237-1245.

(11) Tao, W.; Levine, A. J. Nucleocytoplasmic shuttling of oncoprotein Hdm2 is required for Hdm2-mediated degradation of p53. Proceedings of the National Academy of Sciences of the United States of America 1999, 96, 3077-3080.

(12) Kussie, P. H.; Gorina, S.; Marechal, V.; Elenbaas, B.; Moreau, J.; Levine, A. J.; Pavletich, N. P. Structure of the MDM2 Oncoprotein Bound to the p53 Tumor Suppressor Transactivation Domain. Science 1996, 274, 948-953. 
(13) Honda, R.; Tanaka, H.; Yasuda, H. Oncoprotein MDM2 is a ubiquitin ligase E3 for tumor suppressor p53. FEBS letters 1997, 420, 25-27.

(14) Wells, M.; Tidow, H.; Rutherford, T. J.; Markwick, P.; Jensen, M. R.; Mylonas, E.; Svergun, D. I.; Blackledge, M.; Fersht, A. R. Structure of tumor suppressor p53 and its intrinsically disordered N-terminal transactivation domain. Proceedings of the National Academy of Sciences of the United States of America 2008, 105, 5762-5767.

(15) Rew, Y. et al. Structure-Based Design of Novel Inhibitors of the MDM2-p53 Interaction. Journal of Medicinal Chemistry 2012, 55, 4936-4954.

(16) Estrada-Ortiz, N.; Neochoritis, C. G.; Dömling, A. How To Design a Successful p53MDM2/X Interaction Inhibitor: A Thorough Overview Based on Crystal Structures. ChemMedChem 2015, 11, 757-772.

(17) Baek, S.; Kutchukian, P. S.; Verdine, G. L.; Huber, R.; Holak, T. A.; Lee, K. W.; Popowicz, G. M. Structure of the Stapled p53 Peptide Bound to Mdm2. Journal of the American Chemical Society 2011, 134, 103-106.

(18) Chang, Y. S. et al. Stapled $\alpha$ helical peptide drug development: A potent dual inhibitor of MDM2 and MDMX for p53-dependent cancer therapy. Proceedings of the National Academy of Sciences 2013, 110, E3445-E3454.

(19) Kritzer, J. A.; Lear, J. D.; Hodsdon, M. E.; Schepartz, A. Helical $\beta$-Peptide Inhibitors of the p53-hDM2 Interaction. Journal of the American Chemical Society 2004, 126, 9468-9469.

(20) Robinson, J. a.; DeMarco, S.; Gombert, F.; Moehle, K.; Obrecht, D. The design, structures and therapeutic potential of protein epitope mimetics. Drug Discovery Today 2008, 13, 944-951. 
(21) Robinson, J. a. $\beta$-Hairpin Peptidomimetics: Design, Structures and Biological Activities. Accounts of Chemical Research 2008, 41, 1278-1288.

(22) Fasan, R.; Dias, R. L. A.; Moehle, K.; Zerbe, O.; Vrijbloed, J. W.; Obrecht, D.; Robinson, J. a. Using a $\beta$-Hairpin To Mimic an $\alpha$-Helix: Cyclic Peptidomimetic Inhibitors of the p53-HDM2 Protein-Protein Interaction. Angewandte Chemie 2004, 116, 21612164.

(23) Fasan, R.; Dias, R. L. A.; Moehle, K.; Zerbe, O.; Obrecht, D.; Mittl, P. R. E.; Grütter, M. G.; Robinson, J. a. Structure-Activity Studies in a Family of $\beta$-Hairpin Protein Epitope Mimetic Inhibitors of the p53-HDM2 Protein-Protein Interaction. ChemBioChem 2006, 7, 515-526.

(24) Pazgier, M.; Liu, M.; Zou, G.; Yuan, W.; Li, C.; Li, C.; Li, J.; Monbo, J.; Zella, D.; Tarasov, S. G.; Lu, W. Structural basis for high-affinity peptide inhibition of p53 interactions with MDM2 and MDMX. Proceedings of the National Academy of Sciences of the United States of America 2009, 106, 4665-4670.

(25) Liu, M.; Pazgier, M.; Li, C.; Yuan, W.; Li, C.; Lu, W. A Left-Handed Solution to Peptide Inhibition of the p53-MDM2 Interaction. Angewandte Chemie International Edition 2010, 49, 3649-3652.

(26) Zhan, C.; Zhao, L.; Wei, X.; Wu, X.; Chen, X.; Yuan, W.; Lu, W.-Y.; Pazgier, M.; Lu, W. An Ultrahigh Affinity d-Peptide Antagonist Of MDM2. Journal of Medicinal Chemistry 2012, 55, 6237-6241.

(27) Mas-Moruno, C.; Rechenmacher, F.; Kessler, H. Cilengitide: the first anti-angiogenic small molecule drug candidate design, synthesis and clinical evaluation. Anti-cancer agents in medicinal chemistry 2010, 10, 753-768.

(28) Syud, F. A.; Stanger, H. E.; Gellman, S. H. Interstrand Side ChainSide Chain Inter- 
actions in a Designed $\beta$-Hairpin: Significance of Both Lateral and Diagonal Pairings. Journal of the American Chemical Society 2001, 123, 8667-8677.

(29) n Gago, P. M.; Ramón, R.; Aragón, E.; Fernández-Carneado, J.; Martin-Malpartida, P.; Verdaguer, X.; López-Ruiz, P.; Colás, B.; Cortes, M. A.; Ponsati, B.; Macias, M. J.; Riera, A. A tetradecapeptide somatostatin dicarba-analog: Synthesis, structural impact and biological activity. Bioorganic \&3 Medicinal Chemistry Letters 2014, 24, 103-107.

(30) Lama, D.; Quah, S. T.; Verma, C. S.; Lakshminarayanan, R.; Beuerman, R. W.; Lane, D. P.; Brown, C. J. Rational Optimization of Conformational Effects Induced By Hydrocarbon Staples in Peptides and their Binding Interfaces. Scientific reports 2013, 3, 277-10.

(31) Saglam, A. S.; Wang, D. W.; Zwier, M. C.; Chong, L. T. Flexibility vs Preorganization: Direct Comparison of Binding Kinetics for a Disordered Peptide and Its Exact Preorganized Analogues. The Journal of Physical Chemistry B 2017, 121, 10046-10054.

(32) Chatterjee, D.; Boyd, C. D.; O'Toole, G. A.; Sondermann, H. Structural Characterization of a Conserved, Calcium-Dependent Periplasmic Protease from Legionella pneumophila. Journal of Bacteriology 2012, 194, 4415-4425.

(33) Brown, C. J.; Quah, S. T.; Jong, J.; Goh, A. M.; Chiam, P. C.; Khoo, K. H.; Choong, M. L.; Lee, M. A.; Yurlova, L.; Zolghadr, K.; Joseph, T. L.; Verma, C. S.; Lane, D. P. Stapled Peptides with Improved Potency and Specificity That Activate p53. ACS Chemical Biology 2013, 8, 506-512.

(34) Schafmeister, C. E.; Po, J.; Verdine, G. L. An All-Hydrocarbon Cross-Linking System for Enhancing the Helicity and Metabolic Stability of Peptides. Journal of the American Chemical Society 2000, 122, 5891-5892.

(35) Walensky, L. D.; Bird, G. H. Hydrocarbon-Stapled Peptides: Principles, Practice, and Progress. Journal of Medicinal Chemistry 2014, 57, 6275-6288. 
(36) Edwards, A. L.; Gavathiotis, E.; LaBelle, J. L.; Braun, C. R.; Opoku-Nsiah, K. A.; Bird, G. H.; Walensky, L. D. Multimodal interaction with BCL-2 family proteins underlies the proapoptotic activity of PUMA BH3. Chemistry \&6 biology 2013, 20, 888-902.

(37) Borcherds, W.; Theillet, F.-X.; Katzer, A.; Finzel, A.; Mishall, K. M.; Powell, A. T.; Wu, H.; Manieri, W.; Dieterich, C.; Selenko, P.; Loewer, A.; Daughdrill, G. W. Disorder and residual helicity alter p53-Mdm2 binding affinity and signaling in cells. Nature chemical biology 2014, 10, 1000-1002.

(38) Zhou, G.; Pantelopulos, G. A.; Mukherjee, S.; Voelz, V. A. Bridging Microscopic and Macroscopic Mechanisms of p53-MDM2 Binding with Kinetic Network Models. Biophysj 2017, 113, 785-793.

(39) Paul, F.; Noé, F.; Weikl, T. R. Identifying Conformational-Selection and InducedFit Aspects in the Binding-Induced Folding of PMI from Markov State Modeling of Atomistic Simulations. The Journal of Physical Chemistry B 2018, 122, 5649-5656.

(40) Danelius, E.; Pettersson, M.; Bred, M.; Min, J.; Waddell, M. B.; Guy, R. K.; Grøtli, M.; Erdelyi, M. Flexibility is important for inhibition of the MDM2/p53 protein-protein interaction by cyclic $\beta$-hairpins. Org. Biomol. Chem. 2016, 14, 10386-10393.

(41) Cicero, D. O.; Barbato, G.; Bazzo, R. NMR Analysis of Molecular Flexibility in Solution: A New Method for the Study of Complex Distributions of Rapidly Exchanging Conformations. Application to a 13-Residue Peptide with an 8-Residue Loop. Journal of the American Chemical Society 1995, 117, 1027-1033.

(42) Wang, J.; Wolf, R. M.; Caldwell, J. W.; Kollman, P. A.; Case, D. a. Development and testing of a general amber force field. Journal of Computational Chemistry 2004, 25, $1157-1174$.

(43) Jakalian, A.; Jack, D. B.; Bayly, C. I. Fast, efficient generation of high-quality atomic 
charges. AM1-BCC model: II. Parameterization and validation. Journal of Computational Chemistry 2002, 23, 1623-1641.

(44) Sousa da Silva, A. W.; Vranken, W. F. ACPYPE - AnteChamber PYthon Parser interfacE. BMC research notes 2012, 5, 367.

(45) Pronk, S.; Páll, S.; Schulz, R.; Larsson, P.; Bjelkmar, P.; Apostolov, R.; Shirts, M. R.; Smith, J. C.; Kasson, P. M.; van der Spoel, D.; Hess, B.; Lindahl, E. GROMACS 4.5: a high-throughput and highly parallel open source molecular simulation toolkit. Bioinformatics 2013, 29, 845-854.

(46) Shirts, M.; Pande, V. S. COMPUTING: Screen Savers of the World Unite! Science 2000, 290, 1903-1904.

(47) Li, D.-W.; Brüschweiler, R. NMR-Based Protein Potentials. Angewandte Chemie International Edition 2010, 49, 6778-6780.

(48) Beauchamp, K. A.; McGibbon, R.; Lin, Y.-S.; Pande, V. S. Simple few-state models reveal hidden complexity in protein folding. Proceedings of the National Academy of Sciences of the United States of America 2012, 109, 17807-17813.

(49) Acharyya, A.; Ge, Y.; Wu, H.; DeGrado, W. F.; Voelz, V. A.; Gai, F. Exposing the Nucleation Site in $\alpha$-Helix Folding: A Joint Experimental and Simulation Study. The Journal of Physical Chemistry B 2019, 123, 1797-1807.

(50) Harrigan, M. P.; Sultan, M. M.; Hernández, C. X.; Husic, B. E.; Eastman, P.; Schwantes, C. R.; Beauchamp, K. A.; Mcgibbon, R. T.; Pande, V. S. MSMBuilder: Statistical Models for Biomolecular Dynamics. Biophysj 2017, 112, 10-15.

(51) Scherer, M. K.; Trendelkamp-Schroer, B.; Paul, F.; Pérez-Hernández, G.; Hoffmann, M.; Plattner, N.; Wehmeyer, C.; Prinz, J.-H.; Noé, F. PyEMMA 2: A Software Package for 
Estimation, Validation, and Analysis of Markov Models. Journal of Chemical Theory and Computation 2015, 11, 5525-5542.

(52) Pérez-Hernández, G.; Paul, F.; Giorgino, T.; De Fabritiis, G.; Noé, F. Identification of slow molecular order parameters for Markov model construction. The Journal of Chemical Physics 2013, 139, 015102-14.

(53) Schwantes, C. R.; Pande, V. S. Improvements in Markov State Model Construction Reveal Many Non-Native Interactions in the Folding of NTL9. Journal of Chemical Theory and Computation 2013, 9, 2000-2009.

(54) Mcgibbon, R. T.; Pande, V. S. Variational cross-validation of slow dynamical modes in molecular kinetics. The Journal of Chemical Physics 2015, 142, 124105-13.

(55) Razavi, A. M.; Wuest, W. M.; Voelz, V. A. Computational Screening and Selection of Cyclic Peptide Hairpin Mimetics by Molecular Simulation and Kinetic Network Models. Journal of Chemical Information and Modeling 2014, 54, 1425-1432.

(56) Razavi, A. M.; Voelz, V. A. Kinetic Network Models of Tryptophan Mutations in $\beta$-Hairpins Reveal the Importance of Non-Native Interactions. Journal of Chemical Theory and Computation 2015, 11, 2801-2812.

(57) Ge, Y.; Kier, B. L.; Andersen, N. H.; Voelz, V. A. Computational and Experimental Evaluation of Designed $\beta$-Cap Hairpins Using Molecular Simulations and Kinetic Network Models. Journal of Chemical Information and Modeling 2017, 57, 1609-1620.

(58) Ge, Y.; Borne, E.; Stewart, S.; Hansen, M. R.; Arturo, E. C.; Jaffe, E. K.; Voelz, V. A. Simulations of the regulatory ACT domain of human phenylalanine hydroxylase (PAH) unveil its mechanism of phenylalanine binding. Journal of Biological Chemistry 2018, 293, 19532-19543. 
(59) Metzner, P.; Schütte, C.; Vanden-Eijnden, E. Transition Path Theory for Markov Jump Processes. Multiscale Modeling \& Simulation 2009, 7, 1192-1219.

(60) Noé, F.; Schütte, C.; Vanden-Eijnden, E.; Reich, L.; Weikl, T. R. Constructing the equilibrium ensemble of folding pathways from short off-equilibrium simulations. Proceedings of the National Academy of Sciences of the United States of America 2009, 106, 19011-19016.

(61) Berezhkovskii, A.; Hummer, G.; Szabo, A. Reactive flux and folding pathways in network models of coarse-grained protein dynamics. The Journal of Chemical Physics 2009, 130, 205102-6.

(62) Wu, H.; Paul, F.; Wehmeyer, C.; Noé, F. Multiensemble Markov models of molecular thermodynamics and kinetics. Proceedings of the National Academy of Sciences of the United States of America 2016, 113, E3221-30.

(63) Paul, F.; Wehmeyer, C.; Abualrous, E. T.; Wu, H.; Crabtree, M. D.; Schöneberg, J.; Clarke, J.; Freund, C.; Weikl, T. R.; Noé, F. Protein-peptide association kinetics beyond the seconds timescale from atomistic simulations. Nature Communications 2017, 8, 1095.

(64) Mcgibbon, R. T.; Beauchamp, K. A.; Harrigan, M. P.; Klein, C.; Swails, J. M.; Hernández, C. X.; Schwantes, C. R.; Wang, L.-P.; Lane, T. J.; Pande, V. S. MDTraj: A Modern Open Library for the Analysis of Molecular Dynamics Trajectories. Biophysj 2015, 109, 1528-1532.

(65) Koivisto, J. J.; Kumpulainen, E. T. T.; Koskinen, A. M. P. Conformational ensembles of flexible beta-turn mimetics in DMSO-d6. Org. Biomol. Chem. 2010, 8, 2103-2116.

(66) Thepchatri, P.; Eliseo, T.; Cicero, D. O.; Myles, D.; Snyder, J. P. Relationship Among Ligand Conformations in Solution, in the Solid State, and at the Hsp90 Binding Site: 
Geldanamycin and Radicicol. Journal of the American Chemical Society 2007, 129, $3127-3134$.

(67) Danelius, E.; G, O. R.; Mukandlia Mulumba, A. H.; Ong, H.; S, C.; Erdelyi, M.; Lubell, W. D. Dynamic Chirality in the Mechanism of Action of Allosteric CD36 Modulators of Macrophage-Driven Inflammation. J. Med. Chem. 2019, 62, 11071-11079.

(68) E, D.; Danelius, E.; Mitchell, S. A.; F, H. D.; Erdelyi, M.; T, T. A. A Chemical Biology Approach to Under.standing Molecular Recognition of Lipid II by Nisin(1-12): Synthesis and NMR Ensemble Analysis of Nisin(1-12) and Analogues. Chem Eur. J. 2019, 25, 14572-14582.

(69) Thepchatri, P.; Cicero, D. O.; Monteagudo, E.; Ghosh, A. K.; Cornett, B.; Weeks, E. R.; Snyder, J. P. Conformations of Laulimalide in DMSO-d6. Journal of the American Chemical Society 2005, 127, 12838-12846.

(70) Erdelyi, M.; B, P.; Haunstein, K.; Forher, J.; Gertsch, J.; Altman, K.-H.; Carlomagno, T. Conformational Preferences of Natural and C3-Modified Epothilones in Aqueous Solution. J. Med. Chem 2008, 51, 1469-1478.

(71) Danelius, E.; Poongavanam, V.; Peintner, S.; Wieske, L. H. E.; Erdelyi, M.; J, K. Solution Conformations Explain the Chameleonic Behaviour of Macrocyclic Drugs. Chem Eur. J. 2020, 26, 5231-5244.

(72) Peng, C.; Y, A.; amd Xu Z, W. J.; ; Poongavanam, V.; Shi, J.; Kihlberg, J.; W, Z.; Erdelyi, M. Conformation of the Macrocyclic Drug Lorlatinib in Polar and Nonpolar Environments: A MD Simulation and NMR Study. ACS Omega 2019, 4, 22245-22250.

(73) Lakdawala, A.; Wang, M.; Nevins, N.; Liotta, D. C.; Rusinska-Roszak, D.; Lozynski, M.; Snyder, J. P. Calculated conformer energies for organic molecules with multiple polar functionalities are method dependent: Taxol (case study). BMC Chem. Biol. 2001, 1, 2. 
(74) Favre, M.; Moehle, K.; Jiang, L.; Pfeiffer, B.; Robinson, J. A. Structural mimicry of canonical conformations in antibody hypervariable loops using cyclic peptides containing a heterochiral diproline template. Journal of the American Chemical Society 1999, 121, 2679-2685.

(75) Nüske, F.; Keller, B. G.; Pérez-Hernández, G.; Mey, A. S. J. S.; Noé, F. Variational Approach to Molecular Kinetics. Journal of Chemical Theory and Computation 2014, 10, 1739-1752.

(76) Bernal, F.; Wade, M.; Godes, M.; Davis, T. N.; Whitehead, D. G.; Kung, A. L.; Wahl, G. M.; Walensky, L. D. A Stapled p53 Helix Overcomes HDMX-Mediated Suppression of p53. Cancer Cell 2010, 18, 411-422.

(77) Guo, Z.; Streu, K.; Krilov, G.; Mohanty, U. Probing the Origin of Structural Stability of Single and Double Stapled p53 Peptide Analogs Bound to MDM2. Chemical Biology E3 Drug Design 2014, 83, 631-642.

(78) Doak, B. C.; Zheng, J.; Dobritzsch, D.; Kihlberg, J. How Beyond Rule of 5 Drugs and Clinical Candidates Bind to Their Targets. Journal of Medicinal Chemistry 2016, 59, 2312-2327.

(79) Kumar, E. A.; Chen, Q.; Kizhake, S.; Kolar, C.; Kang, M.; Chang, C.-e. A.; Borgstahl, G. E. O.; Natarajan, A. The paradox of conformational constraint in the design of $\mathrm{Cbl}(\mathrm{TKB})$-binding peptides. Scientific reports 2013, 3, 677-7.

(80) Akaike, H. In Information Theory and an Extension of the Maximum Likelihood Principle. In B. N. Petrov, F. Csaki (Eds.), Proceedings of the 2nd International Symposium on Information Theory. 1973, 267-281.

(81) Nevins, N.; D, C.; Snyder, J. P. CA Test of the Single-Conformation Hypothesis in the Analysis of NMR Data for Small Polar Molecules: A Force Field Comparison, year 
$=1999$, volume $=64$, number $=11$, pages $=3979-3986$, publisher $=$ ACS, doi $=$ 10.1021 $/$ jo9824450, language $=$ English, abstract $=$ A tricyclic ketone with seven stereogenic centers produced in i95:5 diastereomeric excess by an asymmetric DielsAlder reaction has been subjected to a careful 2-D NMR(CDCl3)/MD analysis by Reggelin and co-workers and interpreted in terms of a single conformation in $\mathrm{CDCl} 3$ solution. The present work examines the validity of this interpretation. Specifically, the conformational profile of the endo isomer of the tricyclic ketone was examined by Monte Carlo searches with both the MM2* and MMFF force fields in MacroModel using the GB/SA $\mathrm{CHCl} 3$ solvent continuum model. The two sets of conformations were then combined and optimized with the MM3(96) force field. The structures of the resulting conformations and the NMR-derived geometric variables were together subjected to a NAMFIS analysis (NMR analysis of molecular flexibility in solution). Ideally, the procedure deconvolutes the thermally averaged NMR data into a small family of conformations that optimally represents the J-derived torsions and the NOE-derived distances. It is concluded that the tricyclic ketone under study is not well-characterized by a single conformation in $\mathrm{CDCl} 3$ solution, but by a set of rapidly equilibrating conformers that produces a deceptively averaged NMR spectrum. It would appear that the previously suggested structure is a virtual conformation obligated to compress multiconformer features into a single 3-D construct. In additon, we find that current force fields do not uniformly represent the conformational profile of polar molecules such as 1 . The cause is traced primarily to the variable treatment of electrostatic effects. ., url $=$ https://pubs.acs.org/doi/abs/10.1021/jo9824450,.

(82) Pongavanam, V.; Danelius, E.; Peintner, S.; Alcaraz, L.; Caron, G.; Cummings, M. D.; Wlodek, S.; Erdelyi, M.; Hawkins, P. C. D.; Ermondi, G.; Kihlberg, J. Conformational Sampling of Macrocyclic Drugs in Different Environments: Can We Find the Relevant Conformations?, year $=2018$, volume $=3$, number $=9$, pages $=11742-11757$, publisher $=$ ACS , doi $=10.1021$ acsomega.8b01379, language $=$ English, abstract $=$ Conforma- 
tional flexibility is a major determinant of the properties of macrocycles and other drugs in beyond rule of 5 (bRo5) space. Prediction of conformations is essential for design of drugs in this space, and we have evaluated three tools for conformational sampling of a set of 10 bRo5 drugs and clinical candidates in polar and apolar environments. The distance-geometry based OMEGA was found to yield ensembles spanning larger structure and property spaces than the ensembles obtained by MOE-LowModeMD (MOE) and MacroModel (MC). Both MC and OMEGA but not MOE generated different ensembles for polar and apolar environments. All three conformational search methods generated conformers similar to the crystal structure conformers for 9 of the 10 compounds, with OMEGA performing somewhat better than MOE and MC. MOE and OMEGA found all six conformers of roxithromycin that were identified by NMR in aqueous solutions, whereas only OMEGA sampled the three conformers observed in chloroform. We suggest that characterization of conformers using molecular descriptors, e.g., the radius of gyration and polar surface area, is preferred to energy- or root-meansquare deviation-based methods for selection of biologically relevant conformers in drug discovery in bRo5 space., url = https://pubs.acs.org/doi/10.1021/acsomega.8b01379,.

(83) Voelz, V. A.; Zhou, G. Bayesian inference of conformational state populations from computational models and sparse experimental observables. Journal of Computational Chemistry 2014, 35, 2215-2224.

(84) Ge, Y.; Voelz, V. A. Model Selection Using BICePs: A Bayesian Approach for Force Field Validation and Parameterization. The Journal of Physical Chemistry B 2018, 122, 5610-5622.

(85) Wan, H.; Ge, Y.; Razavi, A.; Voelz, V. A. Reconciling Simulated Ensembles of Apomyoglobin with Experimental Hydrogen/Deuterium Exchange Data Using Bayesian Inference and Multiensemble Markov State Models. Journal of Chemical Theory and Computation 2020, 16, 1333-1348. 
(86) Copeland, R. A. The drug-target residence time model: a 10-year retrospective. Nature Reviews Drug Discovery 2016, 15, 87-95.

(87) Copeland, R. A.; Pompliano, D. L.; Meek, T. D. Drug-target residence time and its implications for lead optimization. Nature Reviews Drug Discovery 2006, 5, 730-739.

(88) Núñez, S.; Venhorst, J.; Kruse, C. G. Target-drug interactions: first principles and their application to drug discovery. Drug Discovery Today 2012, 17, 10-22.

(89) Lotz, S. D.; Dickson, A. Unbiased Molecular Dynamics of 11 min Timescale Drug Unbinding Reveals Transition State Stabilizing Interactions. Journal of the American Chemical Society 2018, 140, 618-628.

(90) Tiwary, P.; Limongelli, V.; Salvalaglio, M.; Parrinello, M. Kinetics of protein-ligand unbinding: Predicting pathways, rates, and rate-limiting steps. Proceedings of the $\mathrm{Na}$ tional Academy of Sciences of the United States of America 2015, 112, E386-E391.

(91) Bruce, N. J.; Ganotra, G. K.; Kokh, D. B.; Sadiq, S. K.; Wade, R. C. New approaches for computing ligand-receptor binding kinetics. Current Opinion in Structural Biology 2018, 49, 1-10.

(92) Gobbo, D.; Piretti, V.; Di Martino, R. M. C.; Tripathi, S. K.; Giabbai, B.; Storici, P.; Demitri, N.; Girotto, S.; Decherchi, S.; Cavalli, A. Investigating Drug-Target Residence Time in Kinases through Enhanced Sampling Simulations. Journal of Chemical Theory and Computation 2019, 15, 4646-4659. 


\section{Supplementary Information}

\section{Supporting Methods}

\section{Convert experimental $K_{D}$ to theoretical bound state population.}

Given a ligand binding to a protein:

$$
P+L \stackrel{K_{Q}}{\Leftrightarrow} P \cdot L
$$

where $P, L$ and $P \cdot L$ represent protein, ligand and ligand bound to protein, respectively. So that $K_{D}$ can be expressed as:

$$
K_{D}=\frac{[P][L]}{[P \cdot L]}
$$

where brackets are denoted as concentrations. The ratio $\frac{[P]}{[P \cdot L]}$ can be computed as the ratio of unbound states populations and bound states populations. The ligand concentration $[L]$ can be directly computed using the box size of the simulation. Let's denote $\pi_{\text {bound }}$ as bound states populations and $1-\pi_{\text {bound }}$ as unbound states populations, then we can express $K_{D}$ as:

$$
K_{D}=\frac{\left(1-\pi_{\text {bound }}\right)[L]}{\pi_{\text {bound }}}
$$

So that we can compute theoretical $\pi_{\text {bound }}$ using measured $K_{D}$ as:

$$
\pi_{\text {bound }}=\frac{[L]}{K_{D}+[L]}
$$

In this work, $[L]=0.00439 \mathrm{M}$ and measured $K_{D}=129.3 \pm 0.3 \mathrm{nM}$. Thus, the computed $\pi_{\text {bound }}=99.997 \%$. 


\section{A conformational selection model relates $K_{D}$ to preorganization.}

Consider a conformational selection model in which a peptide ligand $L$ can be either in a folded state $F$ or unfolded state $U$, so that $[L]=[F]+[U]$. In this model, the peptide must fold completely before binding to protein $P$ :

$$
P \cdot F \stackrel{K_{D}^{*}}{\Longleftrightarrow} P+F \stackrel{K^{\prime}}{\Leftrightarrow} P+U
$$

Here, $K_{D}^{*}=[P][F] /[P \cdot F]$ is the dissociation constant for a folded (i.e. completely preorganized) peptide, and $K_{u}=[U] /[F]$ is the unfolding equilibrium constant. Thus, the experimental dissociation constant is

$$
\begin{gathered}
K_{D}=\frac{[P][L]}{[P \cdot L]}=\frac{[P]([F]+[U])}{[P \cdot L]}=\frac{[P]\left([F]+K_{u}[F]\right)}{[P \cdot L]}=\frac{[P][F]\left(1+K_{u}\right)}{[P \cdot L]}=K_{D}^{*}\left(1+K_{u}\right) \\
K_{D}=K_{D}^{*}\left(1+K_{u}\right)
\end{gathered}
$$

\section{Supporting Tables}

Table S1: Number of particles and periodic box sizes for ligand only simulations.

\begin{tabular}{l|l|l|l|l|l|l|l|l}
\hline Ligand & $\begin{array}{l}\text { No. } \\
\text { atoms }\end{array}$ & $\begin{array}{l}\mathrm{Na}^{+} \\
\text {atoms }\end{array}$ & $\begin{array}{l}\mathrm{Cl}^{-} \\
\text {atoms }\end{array}$ & $\begin{array}{l}\text { No. water } \\
\text { molecules }\end{array}$ & $\begin{array}{l}\text { Cubic } \\
\text { box } \\
\text { length } \\
(\mathrm{nm})\end{array}$ & $\begin{array}{l}\text { Box size } \\
\left(\mathrm{nm}^{3}\right)\end{array}$ & $\begin{array}{l}\text { Simulation } \\
\text { data }(\mu \mathrm{s})\end{array}$ & $\begin{array}{l}\text { Average } \\
\text { trajectory } \\
\text { length } \\
(\mathrm{ns})\end{array}$ \\
\hline Ligand 1 & 6581 & 7 & 4 & 2131 & 4.069 & 67.37 & 198.65 & 376.94 \\
Ligand 2 & 5080 & 5 & 3 & 1641 & 3.736 & 52.15 & 196.20 & 444.90 \\
Ligand 3 & 5077 & 5 & 3 & 1640 & 3.736 & 52.15 & 196.50 & 464.54 \\
Ligand 4 & 5110 & 4 & 3 & 1647 & 3.738 & 52.23 & 196.96 & 462.35 \\
\hline
\end{tabular}


Table S2: Number of particles and periodic box sizes for bound state ligand/MDM2 simulations.

\begin{tabular}{l|l|l|l|l|l|l|l|l}
\hline Ligand & $\begin{array}{l}\text { No. } \\
\text { atoms }\end{array}$ & $\begin{array}{l}\mathrm{Na}^{+} \\
\text {atoms }\end{array}$ & $\begin{array}{l}\mathrm{Cl}^{-} \\
\text {atoms }\end{array}$ & $\begin{array}{l}\text { No. water } \\
\text { molecules }\end{array}$ & $\begin{array}{l}\text { Cubic } \\
\text { box } \\
\text { length } \\
(\mathrm{nm})\end{array}$ & $\begin{array}{l}\text { Box size } \\
\left(\mathrm{nm}^{3}\right)\end{array}$ & $\begin{array}{l}\text { Simulation } \\
\text { data }(\mu \mathrm{s})\end{array}$ & $\begin{array}{l}\text { Average } \\
\text { trajectory } \\
\text { length } \\
(\mathrm{ns})\end{array}$ \\
\hline Ligand 1 & 37045 & 23 & 23 & 11754 & 7.233 & 378.41 & 184.69 & 237.70 \\
Ligand 2 & 37033 & 23 & 24 & 11759 & 7.236 & 378.87 & 183.71 & 238.27 \\
Ligand 3 & 37111 & 23 & 24 & 11785 & 7.129 & 362.31 & 183.73 & 246.29 \\
Ligand 4 & 37041 & 23 & 25 & 11757 & 7.240 & 379.50 & 182.89 & 242.24 \\
\hline
\end{tabular}

Table S3: Number of particles and periodic box sizes for unbound state ligand/MDM2 simulations.

\begin{tabular}{l|l|l|l|l|l|l|l|l}
\hline Ligand & $\begin{array}{l}\text { No. } \\
\text { atoms }\end{array}$ & $\begin{array}{l}\mathrm{Na}^{+} \\
\text {atoms }\end{array}$ & $\begin{array}{l}\mathrm{Cl}^{-} \\
\text {atoms }\end{array}$ & $\begin{array}{l}\text { No. water } \\
\text { molecules }\end{array}$ & $\begin{array}{l}\text { Cubic } \\
\text { box } \\
\text { length } \\
(\mathrm{nm})\end{array}$ & $\begin{array}{l}\text { Box size } \\
\left(\mathrm{nm}^{3}\right)\end{array}$ & $\begin{array}{l}\text { Simulation } \\
\text { data }(\mu \mathrm{s})\end{array}$ & $\begin{array}{l}\text { Average } \\
\text { trajectory } \\
\text { length } \\
(\mathrm{ns})\end{array}$ \\
\hline Ligand 1 & 37069 & 23 & 23 & 11762 & 7.237 & 379.03 & 1087.91 & 113.24 \\
Ligand 2 & 37072 & 23 & 24 & 11772 & 7.243 & 379.98 & 112.88 & 73.54 \\
Ligand 3 & 37078 & 23 & 24 & 11774 & 7.238 & 379.19 & 113.62 & 72.37 \\
Ligand 4 & 37074 & 23 & 25 & 11768 & 7.236 & 378.87 & 226.04 & 74.77 \\
\hline
\end{tabular}

\section{Supporting Figures}


Table S4: Number of particles and periodic box sizes for peptide1/MDM2 umbrella simulations.

\begin{tabular}{l|l|l|l|l|l|l|l|l}
\hline Ligand & $\begin{array}{l}\text { No. } \\
\text { atoms }\end{array}$ & $\begin{array}{l}\mathrm{Na}^{+} \\
\text {atoms }\end{array}$ & $\begin{array}{l}\mathrm{Cl}^{-} \\
\text {atoms }\end{array}$ & $\begin{array}{l}\text { No. water } \\
\text { molecules }\end{array}$ & $\begin{array}{l}\text { Cubic } \\
\text { box } \\
\text { length } \\
(\mathrm{nm})\end{array}$ & $\begin{array}{l}\text { Box size } \\
\left(\mathrm{nm}^{3}\right)\end{array}$ & $\begin{array}{l}\text { Simulation } \\
\text { data }(\mu \mathrm{s})\end{array}$ & $\begin{array}{l}\text { Average } \\
\text { trajectory } \\
\text { length } \\
(\mathrm{ns})\end{array}$ \\
\hline Ligand 1 & 37045 & 23 & 23 & 11754 & 7.233 & 378.41 & 56.32 & 57.00 \\
\hline
\end{tabular}

Table S5: Interproton distances computed and plotted in Figure 5 for peptide 1.

\begin{tabular}{|c|c|c|c|}
\hline $\begin{array}{l}\text { NOE } \\
\text { index }\end{array}$ & $\begin{array}{l}\text { Interproton } \\
\text { distances }\end{array}$ & $\begin{array}{l}\text { NOE } \\
\text { index }\end{array}$ & $\begin{array}{l}\text { Interproton } \\
\text { distances }\end{array}$ \\
\hline 1 & E2NH-F1NH & 19 & W6NH- ${ }^{D} \mathrm{P} 9 \alpha$ \\
\hline 2 & L4NH-W6NH & 20 & E2NH-F8 $\alpha$ \\
\hline 3 & L4NH-D5NH & 21 & $\mathrm{E} 2 \mathrm{NH}-{ }^{D} \mathrm{P} 9 \alpha$ \\
\hline 4 & $\mathrm{D} 5 \mathrm{NH}-\mathrm{W}(\mathrm{Cl}) 3 \alpha$ & 22 & W6NH-P10 $\alpha$ \\
\hline 5 & W6NH-W(Cl) $3 \alpha$ & 23 & F1NH-W6 $\alpha$ \\
\hline 6 & W6NH-L4 $\alpha$ & 24 & L4NH-P $10 \alpha$ \\
\hline 7 & W6NH-E $2 \alpha$ & 25 & L4NH-E2 $\alpha$ \\
\hline 8 & D 5 NH-L $4 \alpha$ & 26 & D 5 NH-E $2 \alpha$ \\
\hline 9 & W5NH-D $5 \alpha$ & 27 & E2NH-W6 $\alpha$ \\
\hline 10 & F1NH-P10 $\alpha$ & 28 & L4NH-F1NH \\
\hline 11 & $\mathrm{~F} 1 \mathrm{NH}-{ }^{D} \mathrm{P} 9 \alpha$ & 29 & $\mathrm{~F} 1 \mathrm{NH}-\mathrm{W}(\mathrm{Cl}) 3 \alpha$ \\
\hline 12 & $\mathrm{~F} 8 \alpha^{-}{ }^{D} \mathrm{P} 9 \alpha$ & 30 & $\mathrm{~L} 4 \mathrm{NH}-\mathrm{F} 8 \alpha$ \\
\hline 13 & $\mathrm{~W} 6 \alpha-\mathrm{D} 5 \alpha$ & 31 & E2NH-D5NH \\
\hline 14 & $\mathrm{~W}(\mathrm{Cl}) 3 \alpha-\mathrm{L} 4 \alpha$ & 32 & L4NH-W6 $\alpha$ \\
\hline 15 & ${ }^{D} \mathrm{P} 9 \alpha-\mathrm{P} 10 \alpha$ & 33 & W6NH-F1NH \\
\hline 16 & $\mathrm{D} 5 \alpha-\mathrm{L} 4 \alpha$ & 34 & E2NH-L4NH \\
\hline 17 & D5NH-W6 $\alpha$ & 35 & F1NH-D $5 \alpha$ \\
\hline 18 & $\mathrm{E} 2 \alpha-\mathrm{W}(\mathrm{Cl}) 3 \alpha$ & 36 & F1NH-L $4 \alpha$ \\
\hline
\end{tabular}


Table S6: Interproton distances computed and plotted in Figure 5 for peptide 2.

\begin{tabular}{|c|c|c|c|}
\hline $\begin{array}{l}\text { NOE } \\
\text { index }\end{array}$ & $\begin{array}{l}\text { Interproton } \\
\text { distances }\end{array}$ & $\begin{array}{l}\text { NOE } \\
\text { index }\end{array}$ & $\begin{array}{l}\text { Interproton } \\
\text { distances }\end{array}$ \\
\hline 1 & F $8 \mathrm{NH}-{ }^{D} \mathrm{P} 9 \alpha$ & 13 & $\mathrm{~F}(\mathrm{Cl}) 3 \mathrm{NH}-\mathrm{D} 5 \alpha$ \\
\hline 2 & F 8 NH-S $2 \alpha$ & 14 & F1NH-F(Cl)3NH \\
\hline 3 & F8NH-G10NH & 15 & F8NH-E6NH \\
\hline 4 & F8NH-F1NH & 16 & $\mathrm{~F}(\mathrm{Cl}) 3 \mathrm{NH}-\mathrm{D} 5 \mathrm{NH}$ \\
\hline 5 & G10NH-F1NH & 17 & ${ }^{D} \mathrm{P} 9 \alpha-\mathrm{F}(\mathrm{Cl}) 3 \mathrm{NH}$ \\
\hline 6 & G10NH- ${ }^{D} \mathrm{P} 9 \alpha$ & 18 & F8NH-D5NH \\
\hline 7 & $\mathrm{~F}(\mathrm{Cl}) 3 \mathrm{NH}-\mathrm{S} 7 \alpha$ & 19 & F1NH-E6NH \\
\hline 8 & $\mathrm{~F}(\mathrm{Cl}) 3 \mathrm{NH}-\mathrm{E} 6 \mathrm{NH}$ & 20 & $\begin{array}{l}\text { G10NH- } \\
\mathrm{F}(\mathrm{Cl}) 3 \mathrm{NH}\end{array}$ \\
\hline 9 & D5NH-E6NH & 21 & ${ }^{D} \mathrm{P} 9 \alpha-\mathrm{S} 7 \alpha$ \\
\hline 10 & F1NH- ${ }^{D} \mathrm{P} 9 \alpha$ & 22 & ${ }^{D} \mathrm{P} 9 \alpha-\mathrm{S} 2 \alpha$ \\
\hline 11 & E6NH-D5 $\alpha$ & 23 & F8NH-F $(\mathrm{Cl}) 3 \mathrm{NH}$ \\
\hline 12 & $\mathrm{~S} 7 \alpha-\mathrm{S} 2 \alpha$ & 24 & ${ }^{D} \mathrm{P} 9 \alpha-\mathrm{D} 5 \mathrm{NH}$ \\
\hline
\end{tabular}

Table S7: Interproton distances computed and plotted in Figure 5 for peptide 3.

\begin{tabular}{|c|c|c|c|}
\hline $\begin{array}{l}\text { NOE } \\
\text { index }\end{array}$ & $\begin{array}{l}\text { Interproton } \\
\text { distances }\end{array}$ & $\begin{array}{l}\text { NOE } \\
\text { index }\end{array}$ & $\begin{array}{l}\text { Interproton } \\
\text { distances }\end{array}$ \\
\hline 1 & F8NH-S2 $\alpha$ & 18 & F8NH-D5NH \\
\hline 2 & F8NH-F(Br)3NH & 19 & F1NH-D5NH \\
\hline 3 & $\mathrm{~F} 8 \mathrm{NH}_{-}{ }^{D} \mathrm{P} 9 \alpha$ & 20 & ${ }^{D} \mathrm{P} 9 \alpha-\mathrm{E} 6 \mathrm{NH}$ \\
\hline 4 & F8NH-S2NH & 21 & F1NH-E6NH \\
\hline 5 & F8NH-G10NH & 22 & ${ }^{D} \mathrm{P} 9 \alpha-\mathrm{S} 7 \alpha$ \\
\hline 6 & F8NH-F1NH & 23 & $\mathrm{~S} 2 \alpha-\mathrm{S} 7 \alpha$ \\
\hline 7 & S2NH-S7 $\alpha$ & 24 & G10NH-D5NH \\
\hline 8 & G10NH- ${ }^{D} \mathrm{P} 9 \alpha$ & 25 & F8NH-E6NH \\
\hline 9 & G10NH-F1NH & 26 & $\mathrm{~F}(\mathrm{Br}) 3 \mathrm{NH}-{ }^{D} \mathrm{P} 9 \alpha$ \\
\hline 10 & $\mathrm{~F}(\mathrm{Br}) 3 \mathrm{NH}-\mathrm{D} 5 \mathrm{NH}$ & 27 & G10NH-E6NH \\
\hline 11 & $\mathrm{~F}(\mathrm{Br}) 3 \mathrm{NH}-\mathrm{E} 6 \mathrm{NH}$ & 28 & $\mathrm{~S} 2 \alpha_{-}{ }^{D} \mathrm{P} 9 \alpha$ \\
\hline 12 & $\mathrm{~F}(\mathrm{Br}) 3 \mathrm{NH}-\mathrm{S} 7 \alpha$ & 29 & $\mathrm{~S} 2 \alpha-\mathrm{D} 5 \mathrm{NH}$ \\
\hline 13 & $\mathrm{~F}(\mathrm{Br}) 3 \mathrm{NH}-\mathrm{D} 5 \alpha$ & 30 & $\mathrm{~S} 7 \alpha-\mathrm{D} 5 \mathrm{NH}$ \\
\hline 14 & D5NH-E6NH & 31 & $\begin{array}{l}\mathrm{F}(\mathrm{Br}) 3 \mathrm{NH}- \\
\mathrm{G} 10 \mathrm{NH}\end{array}$ \\
\hline 15 & F1NH- ${ }^{D} \mathrm{P} 9 \alpha$ & 32 & ${ }^{D} \mathrm{P} 9 \alpha-\mathrm{S} 2 \mathrm{NH}$ \\
\hline 16 & F1NH-S7 $\alpha$ & 33 & S2NH-E6NH \\
\hline 17 & E6NH-S $2 \alpha$ & 34 & $\mathrm{~F}(\mathrm{Br}) 3 \mathrm{NH}-\mathrm{F} 1 \mathrm{NH}$ \\
\hline
\end{tabular}


Table S8: Interproton distances computed and plotted in Figure 5 for peptide 4.

\begin{tabular}{|c|c|c|c|}
\hline $\begin{array}{l}\text { NOE } \\
\text { index }\end{array}$ & $\begin{array}{l}\text { Interproton } \\
\text { distances }\end{array}$ & $\begin{array}{l}\text { NOE } \\
\text { index }\end{array}$ & $\begin{array}{l}\text { Interproton } \\
\text { distances }\end{array}$ \\
\hline 1 & F8NH-S7NH & 17 & $\mathrm{~W}(\mathrm{Cl}) 3 \mathrm{NH}-{ }^{D} \mathrm{P} 9 \alpha$ \\
\hline 2 & F8NH-F1NH & 18 & S2NH-5D $\alpha$ \\
\hline 3 & F8NH-F1 $\alpha$ & 19 & $\begin{array}{l}\text { F1NH- } \\
\mathrm{W}(\mathrm{Cl}) 3 \mathrm{NH}\end{array}$ \\
\hline 4 & $\begin{array}{l}\text { W(Cl)3NH- } \\
\text { W6NH }\end{array}$ & 20 & $\mathrm{~F} 1 \alpha^{-D} \mathrm{P} 9 \alpha$ \\
\hline 5 & $\mathrm{~W}(\mathrm{Cl}) 3 \mathrm{NH}-\mathrm{S} 2 \mathrm{NH}$ & 21 & W6NH-L4NH \\
\hline 6 & G10NH-F1NH & 22 & $\mathrm{~F} 1 \alpha-\mathrm{W} 6 \mathrm{NH}$ \\
\hline 7 & G10NH- ${ }^{D} \mathrm{P} 9 \alpha$ & 23 & $\mathrm{~F} 1 \alpha-\mathrm{D} 5 \mathrm{NH}$ \\
\hline 8 & L4NH-D5NH & 24 & G10NH-W(Cl)3 $3 \alpha$ \\
\hline 9 & L4NH-W(Cl) $3 \alpha$ & 25 & $\begin{array}{l}\text { W(Cl)3NH- } \\
\text { G10NH }\end{array}$ \\
\hline 10 & S7NH-W6NH & 26 & $\mathrm{~F} 1 \alpha-\mathrm{L} 4 \alpha$ \\
\hline 11 & S2NH-F1NH & 27 & S2NH-L4NH \\
\hline 12 & S2NH-F1 $\alpha$ & 28 & W6NH-L4 $\alpha$ \\
\hline 13 & D5NH-W6NH & 29 & ${ }^{D} \mathrm{P} 9 \alpha-\mathrm{D} 5 \mathrm{NH}$ \\
\hline 14 & D5NH-L4 $\alpha$ & 30 & F8NH-L4NH \\
\hline 15 & W6NH-D $5 \alpha$ & 31 & S7NH-F1 $\alpha$ \\
\hline 16 & F1NH- ${ }^{D} \mathrm{P} 9 \alpha$ & 32 & W6NH-G10NH \\
\hline
\end{tabular}


a

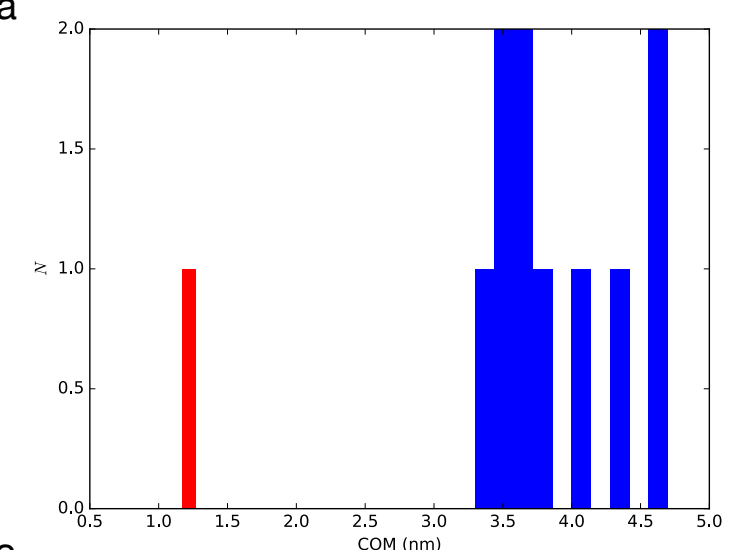

C

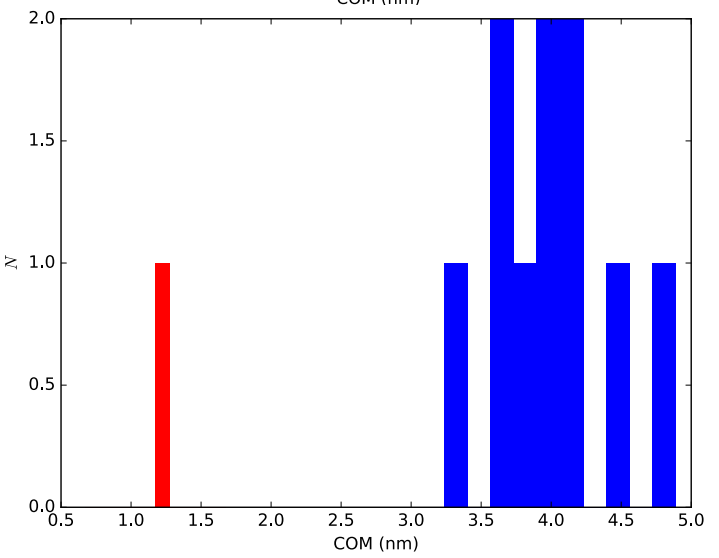

b
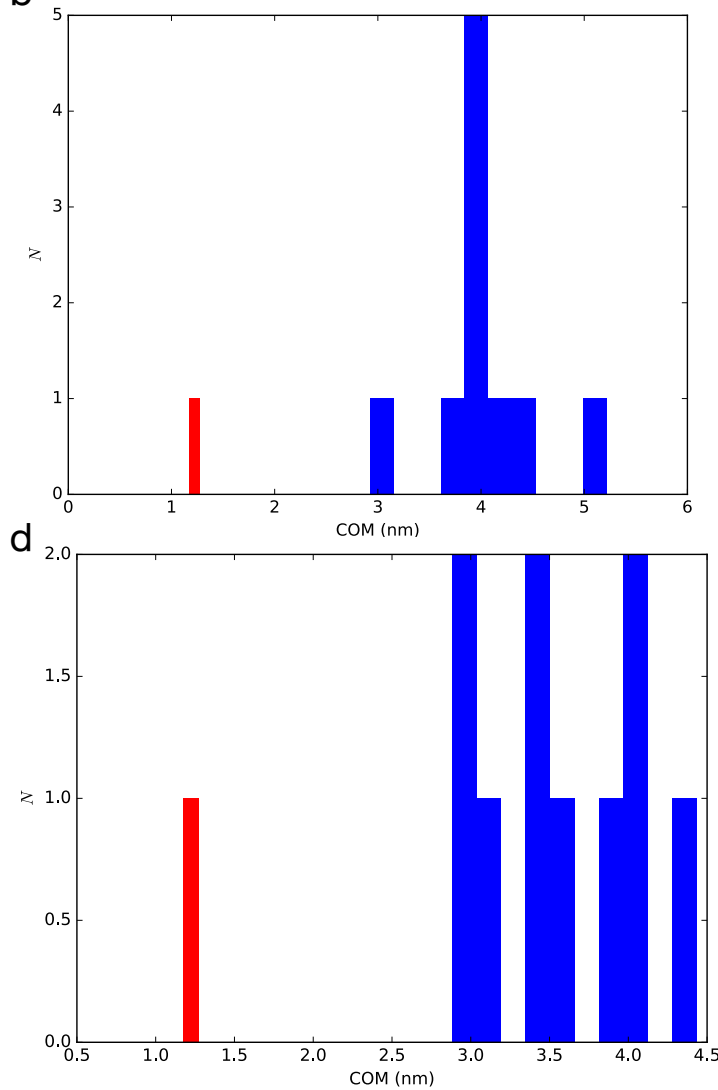

Figure S1: (a-d) Distributions of center of mass distances between ligands and MDM2 in starting points of binding simulations for peptide 1-4. 

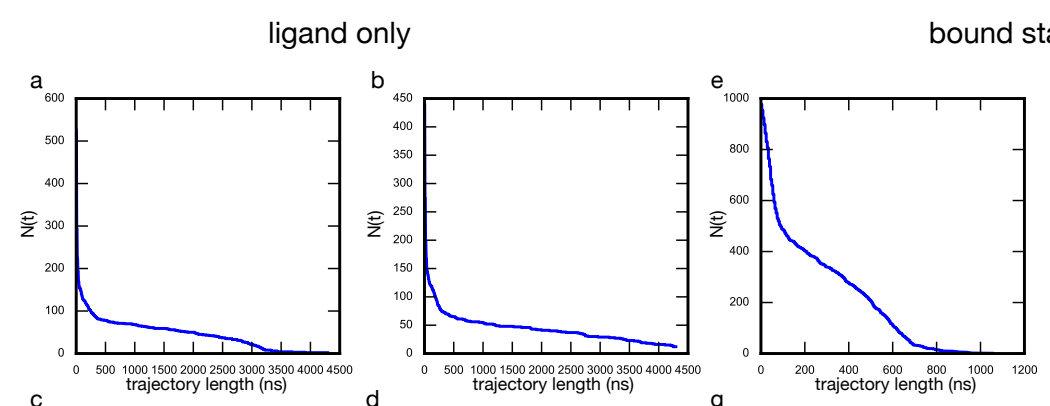

bound state
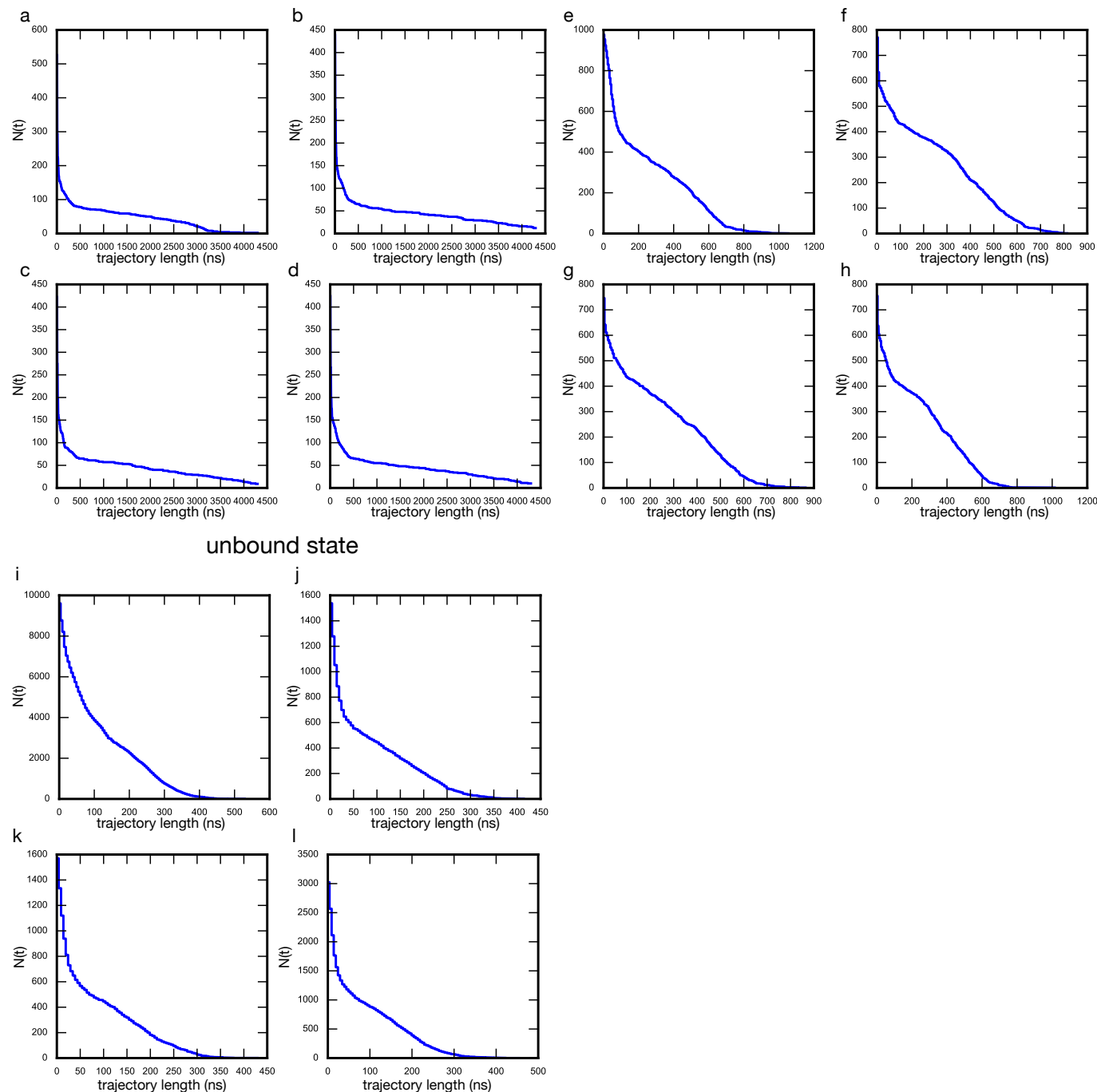

Figure S2: Distributions of trajectory lengths for peptides 1-4 simulations of (a-d) ligand only, (e-h) bound state and (i-l) unbound state, shown as $\mathrm{N}(\mathrm{t})$, the number of trajectories that reach a given length of time, t. (a), (b), (c) and (d) correspond to Ligand 1, 2, 3 and 4 , respectively. 

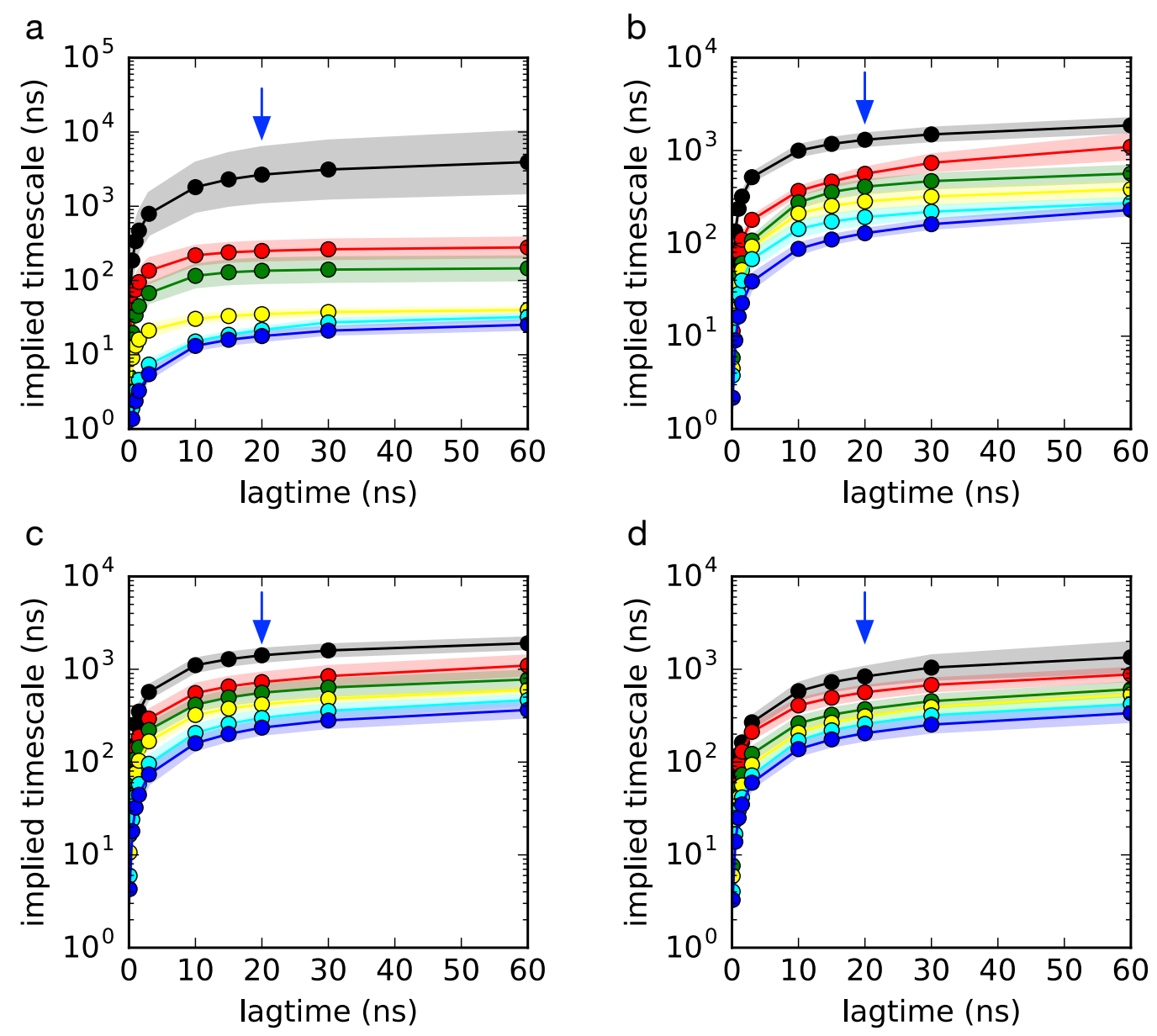

Figure S3: (a-d) Implied timescale plots for peptide 1-4. Shown are implied relaxation times from MSMs built using different lag times. Six slow motions in total are shown in different colored lines. Uncertainty estimates (standard deviations shown as shaded regions) were calculated using a bootstrap procedure, whereby 20 different MSMs were constructed by sampling the input trajectories with replacement. 


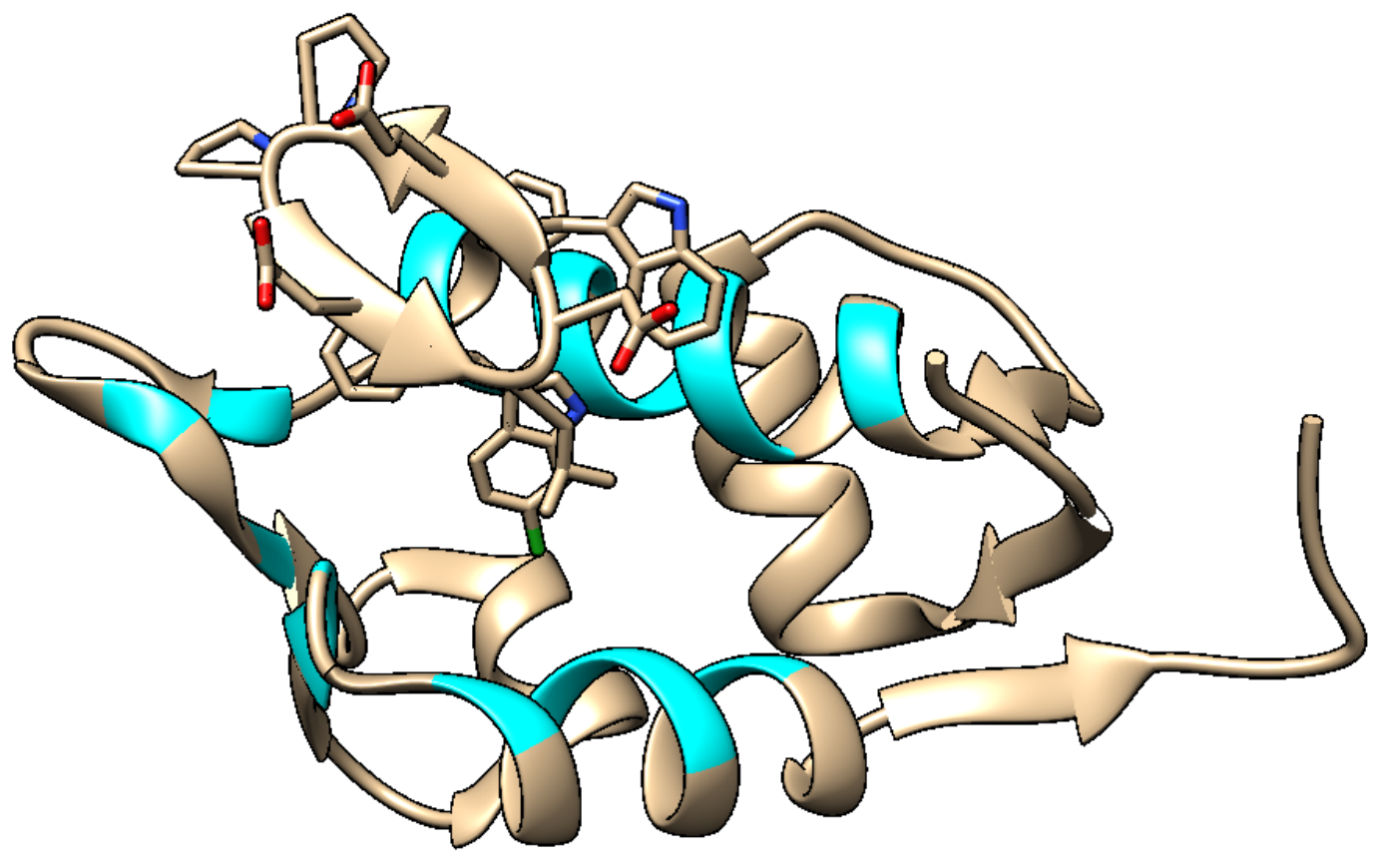

Figure S4: Selected residues on MDM2 to compute pairwise distances as tICA input: Lys51, Leu54, Phe55, Leu57, Gly58, Gln59, Ile61, Met62, Tyr67, Gly72, Val75, Ser92, Val93, His96, Ile99, Tyr100, Ile103. 

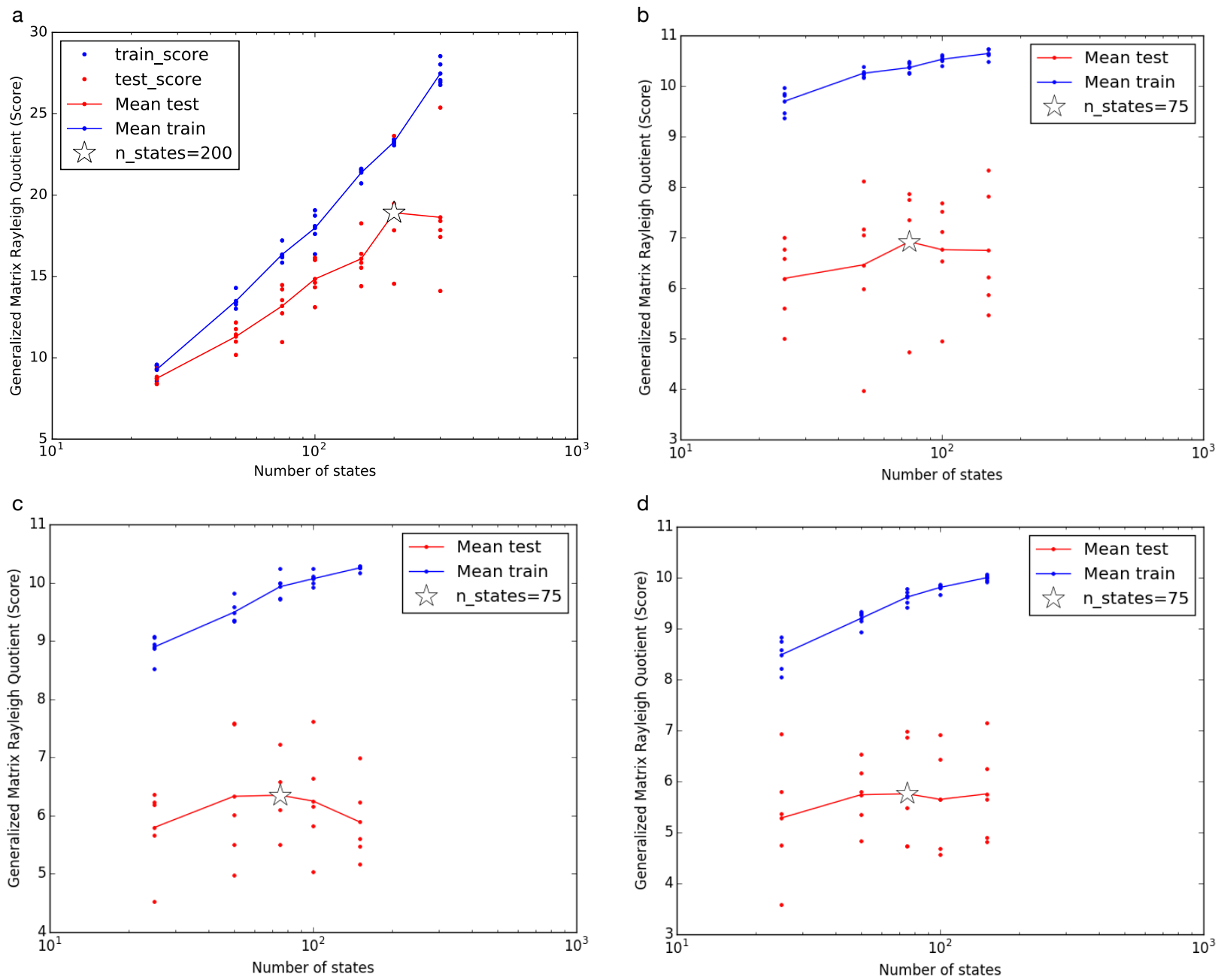

Figure S5: The generalized matrix Rayleigh quotient (GMRQ) method was used to optimize the number of states for constructing a joint MSM of binding and bound state trajectories for peptide 1-4 (a-d). Here, other model construction parameters are held fixed (i.e. 4 tICA components, tICA lag time of $5 \mathrm{~ns}$ ). 


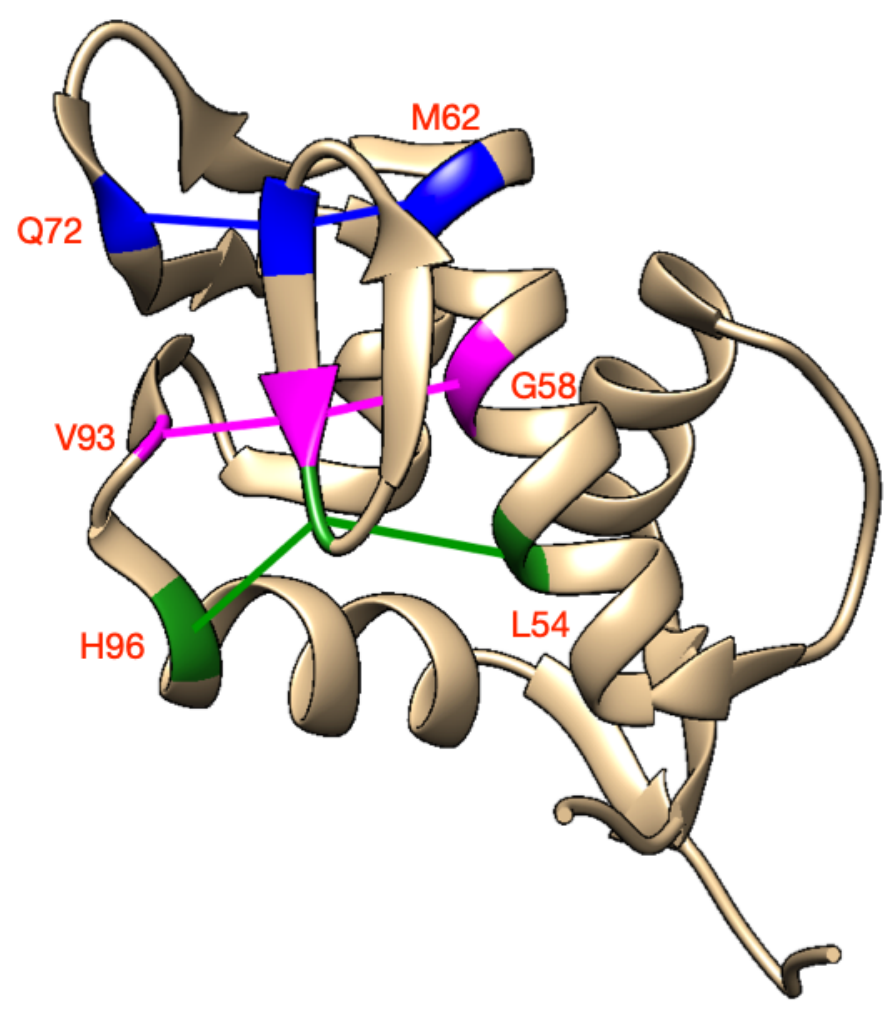

Figure S6: Selected residues on MDM2 and cyclic peptide to compute averaged dis-

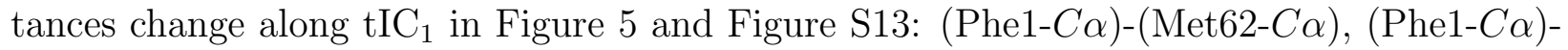
$(\mathrm{Gln} 72-C \alpha), \quad(\operatorname{Trp}(\mathrm{Cl}) 3-C \alpha)-(\operatorname{Val} 93-C \alpha), \quad(\operatorname{Trp}(\mathrm{Cl}) 3-C \alpha)-(\mathrm{Gly} 58-C \alpha), \quad(\mathrm{Leu} 4-C \alpha)-(\mathrm{Leu} 54-$ $C \alpha),($ Leu4-C $\alpha)-($ His96-C $\alpha)$. 


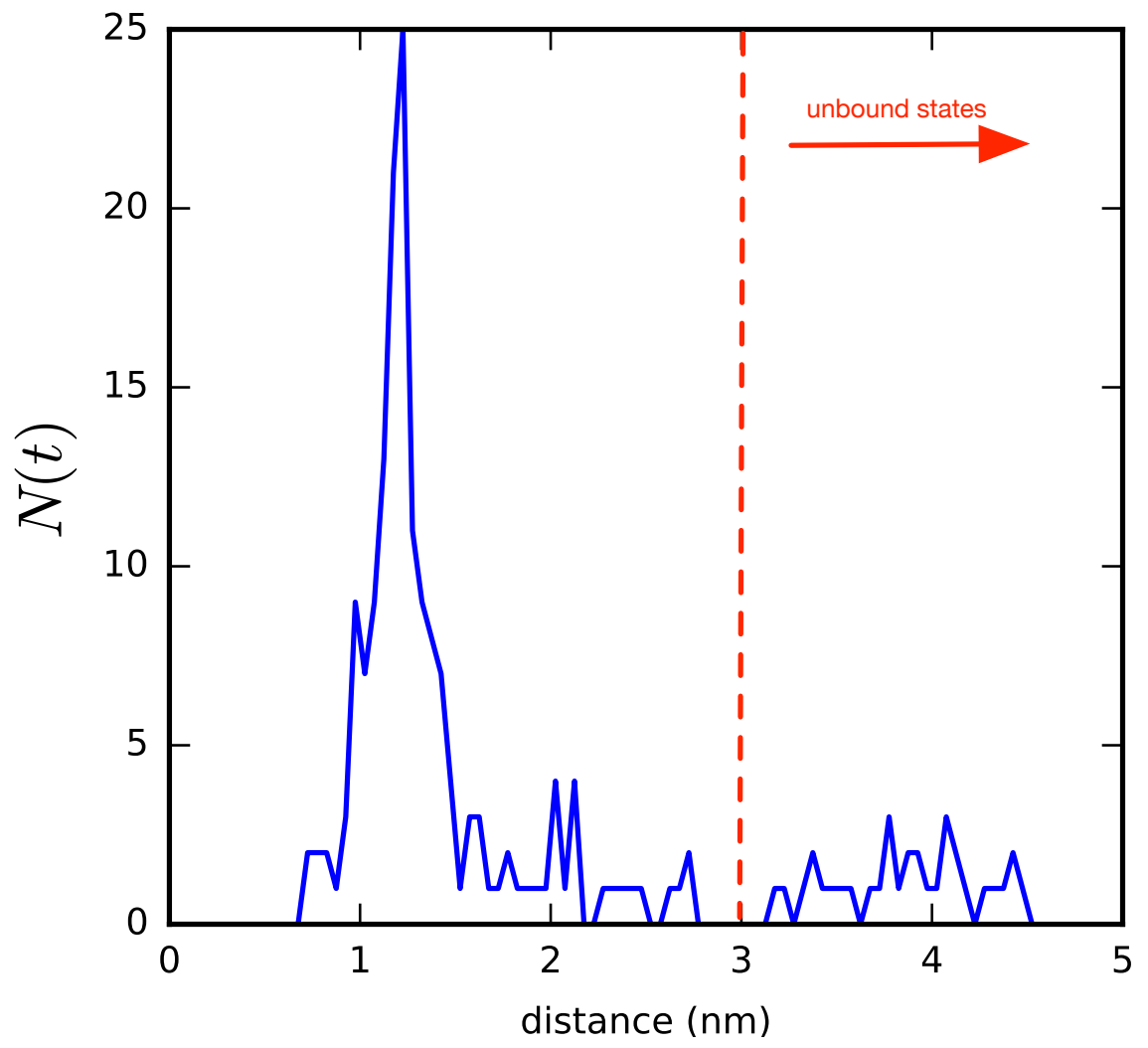

Figure S7: Distribution of state averaged distances between selected residues on peptide 1 and MDM2 (see Figure S6). A cut-off of $3 \mathrm{~nm}$ is used to defined unbound states. 


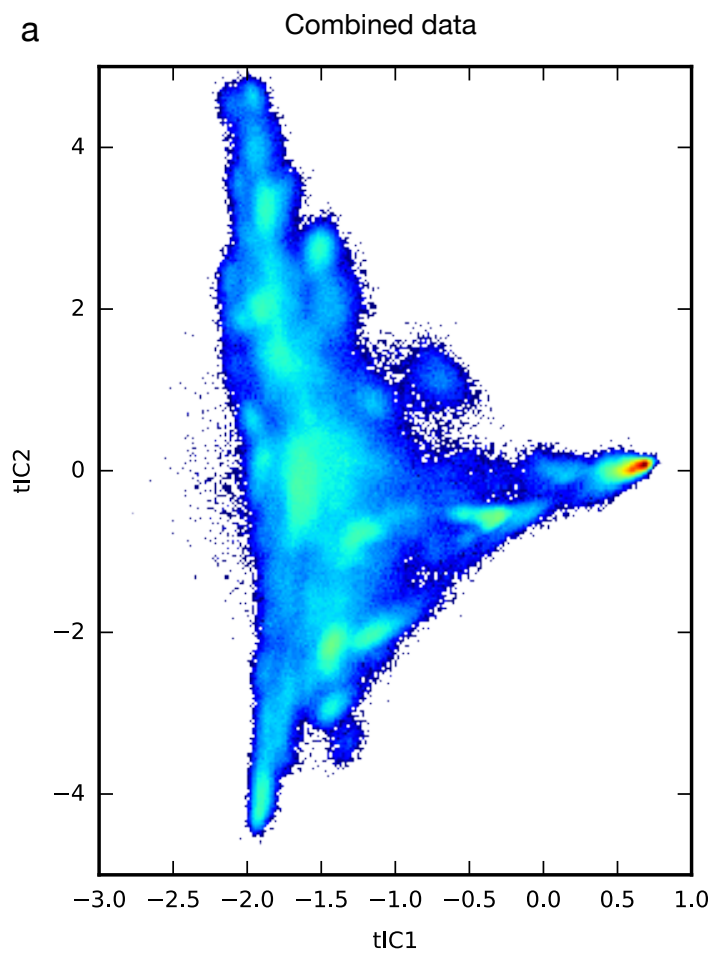

b Binding simulations

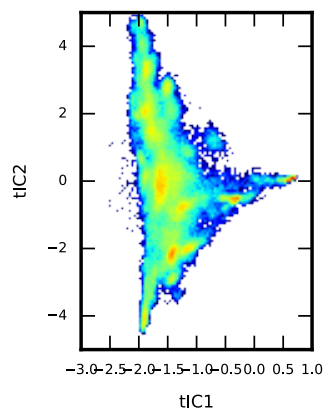

C Bound simulations

d Umbrella simulations
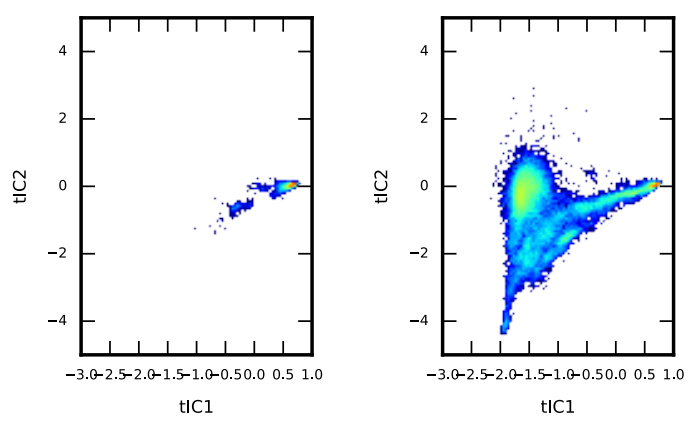

Figure S8: (a) Combination of binding simulations, bound state simulations and umbrella simulations projected to the 2D tICA landscape. Projection of simulation data of (b) binding simulation (c) bound simulation and (d) umbrella simulations alone onto the tICA space from (a). 


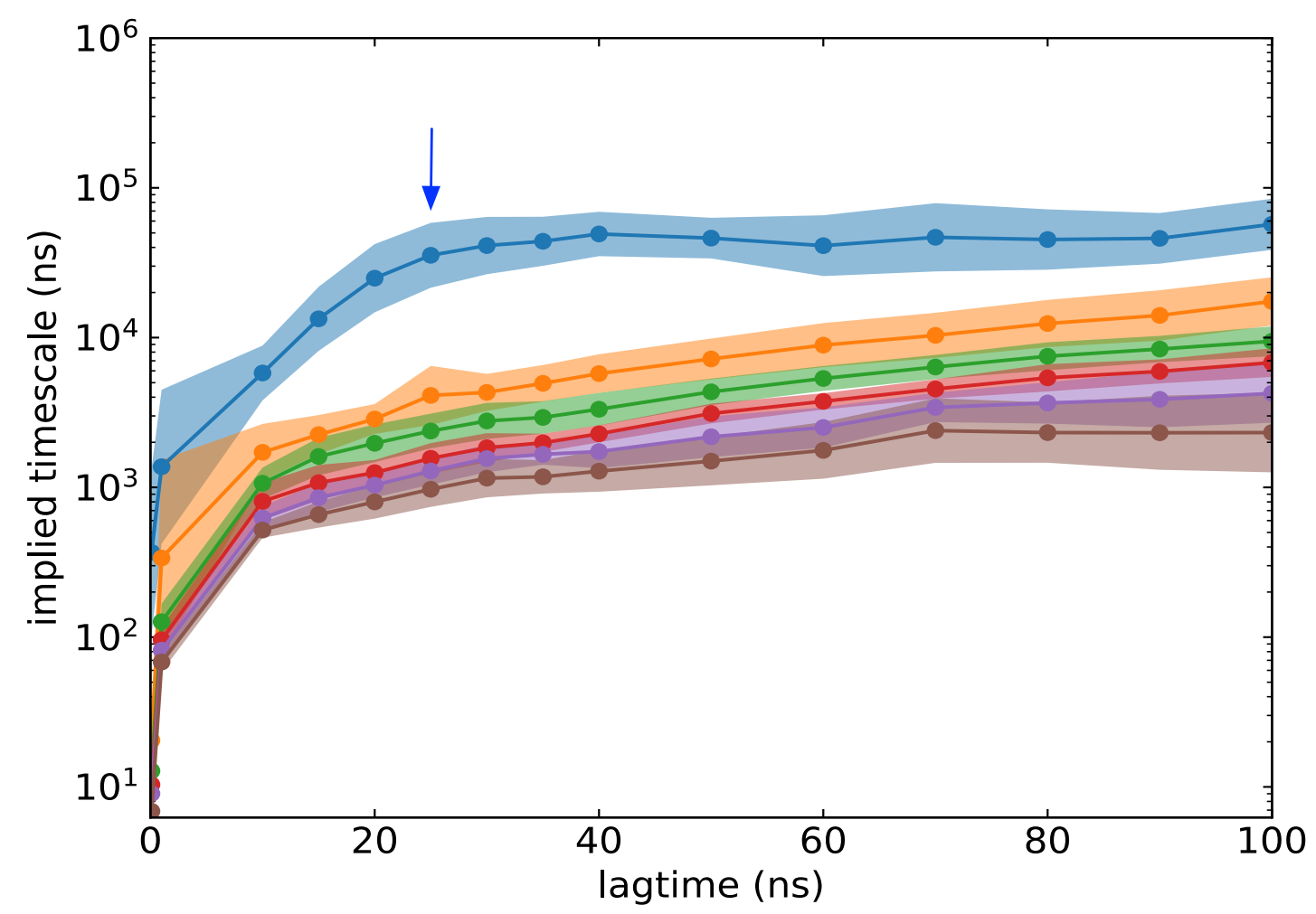

Figure S9: Implied timescale plot for peptide 1. Shown are implied relaxation times from MEMMs built using different lag times. Six slow motions in total are shown in different colored lines. Uncertainty estimates (standard deviations shown as shaded regions) were calculated using a bootstrap procedure, whereby 10 different MEMMs were constructed by sampling the input trajectories of each ensemble with replacement. 

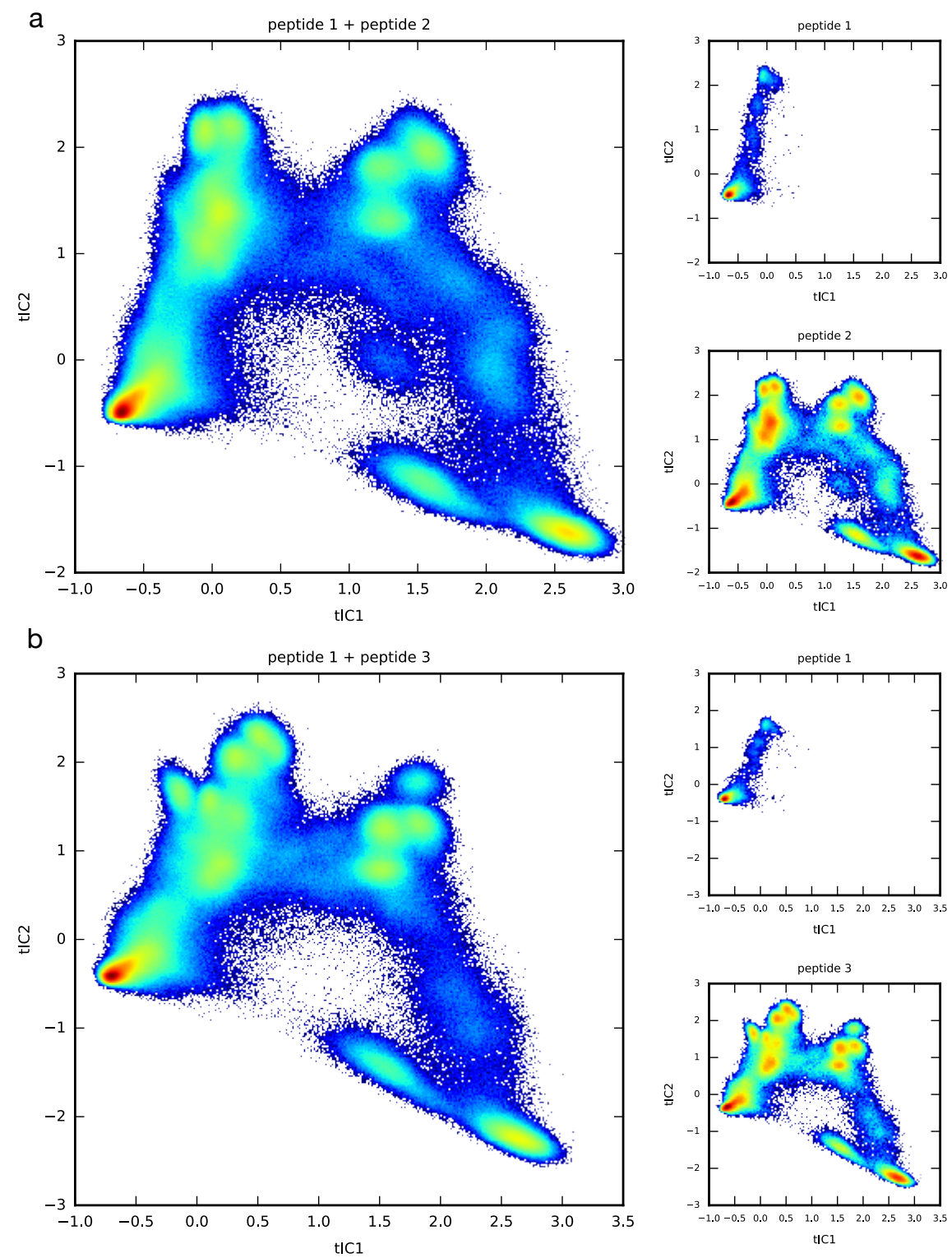

Figure S10: Combination of (a) peptide 1 and 2 and (b) peptide 1 and 3 simulation trajectories projected to the 2D tICA landscape. 

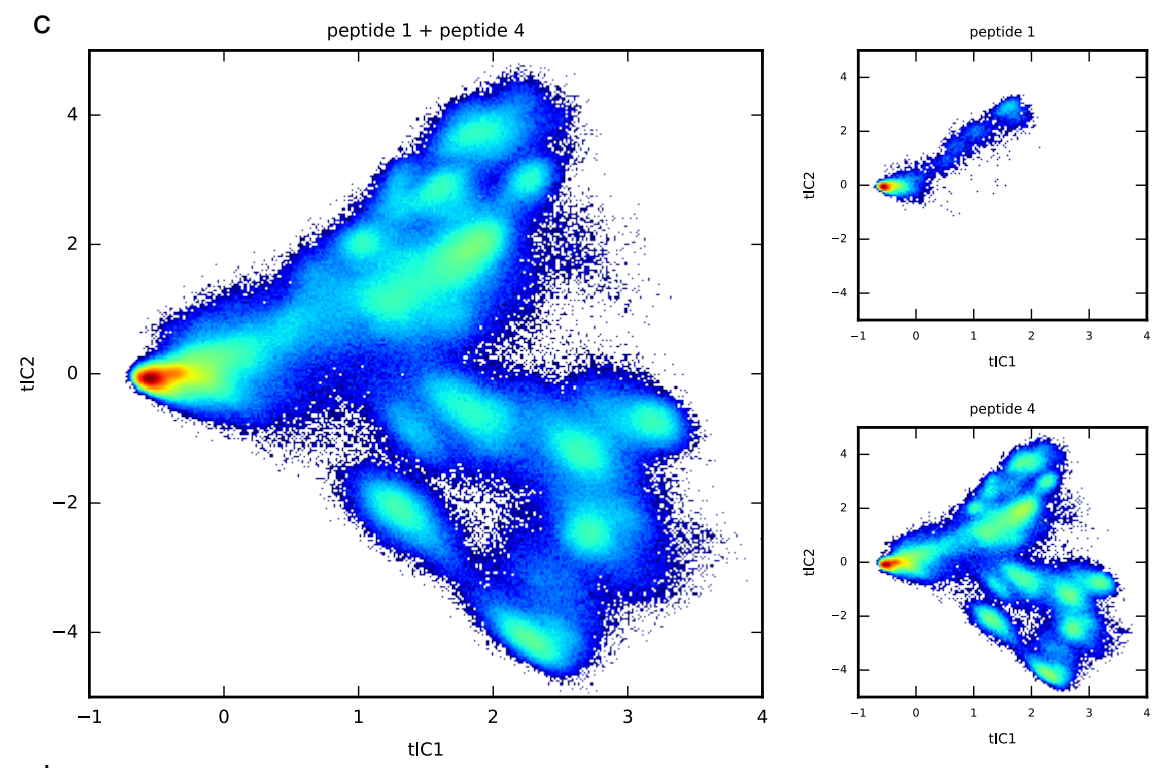

$d$
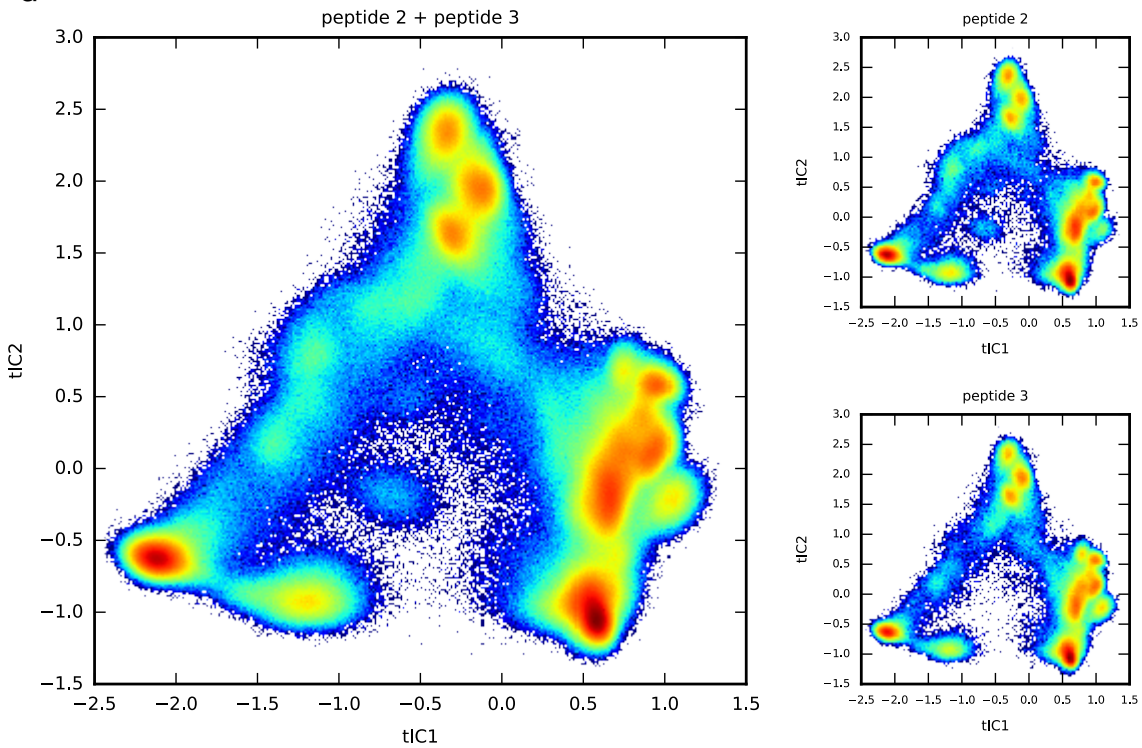

Figure S11: Combination of (c) peptide 1 and 4 and (d) peptide 2 and 3 simulation trajectories projected to the 2D tICA landscape. 

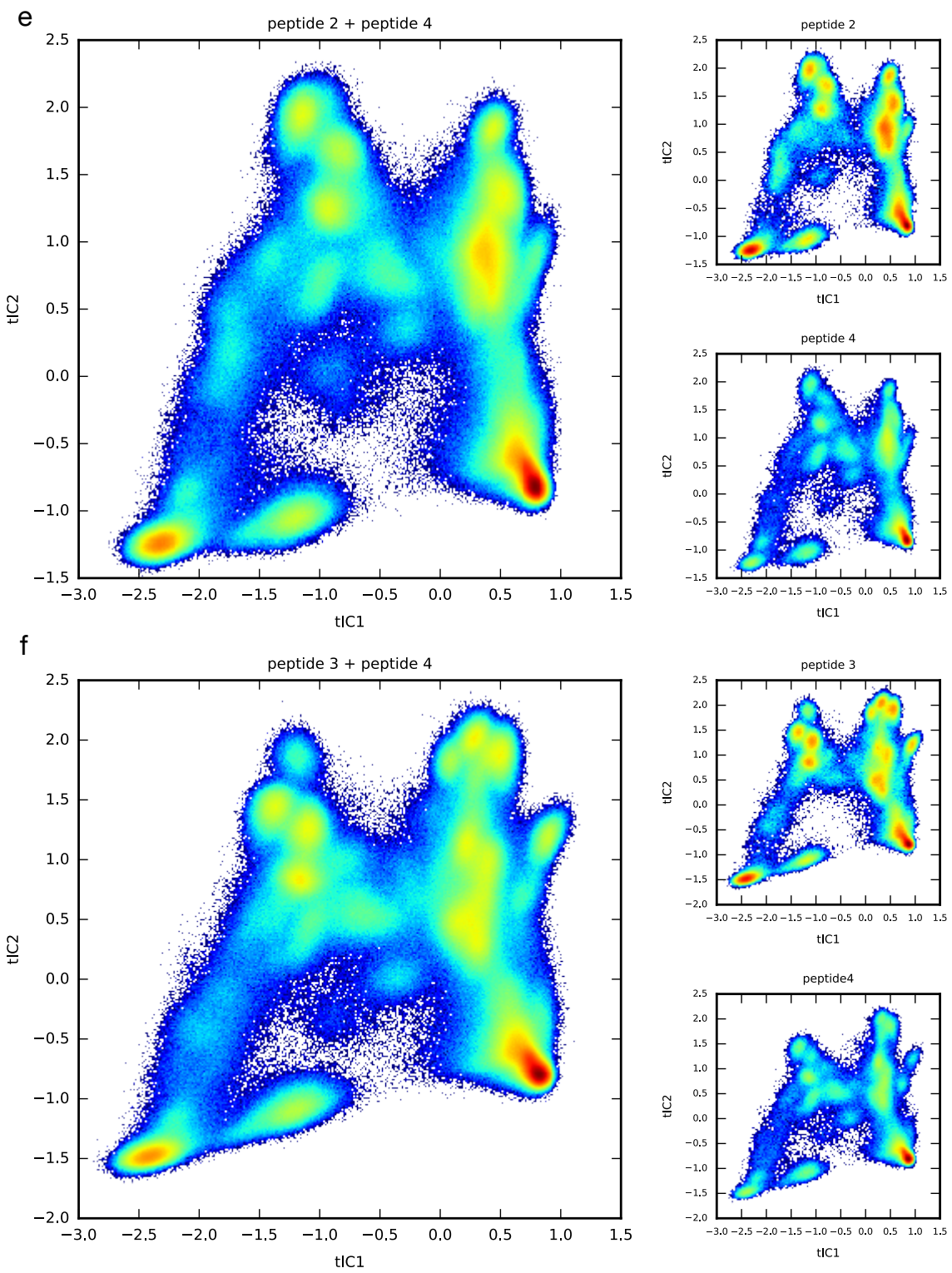

Figure S12: Combination of (e) peptide 2 and 4 and (f) peptide 3 and 4 simulation trajectories projected to the 2D tICA landscape. 

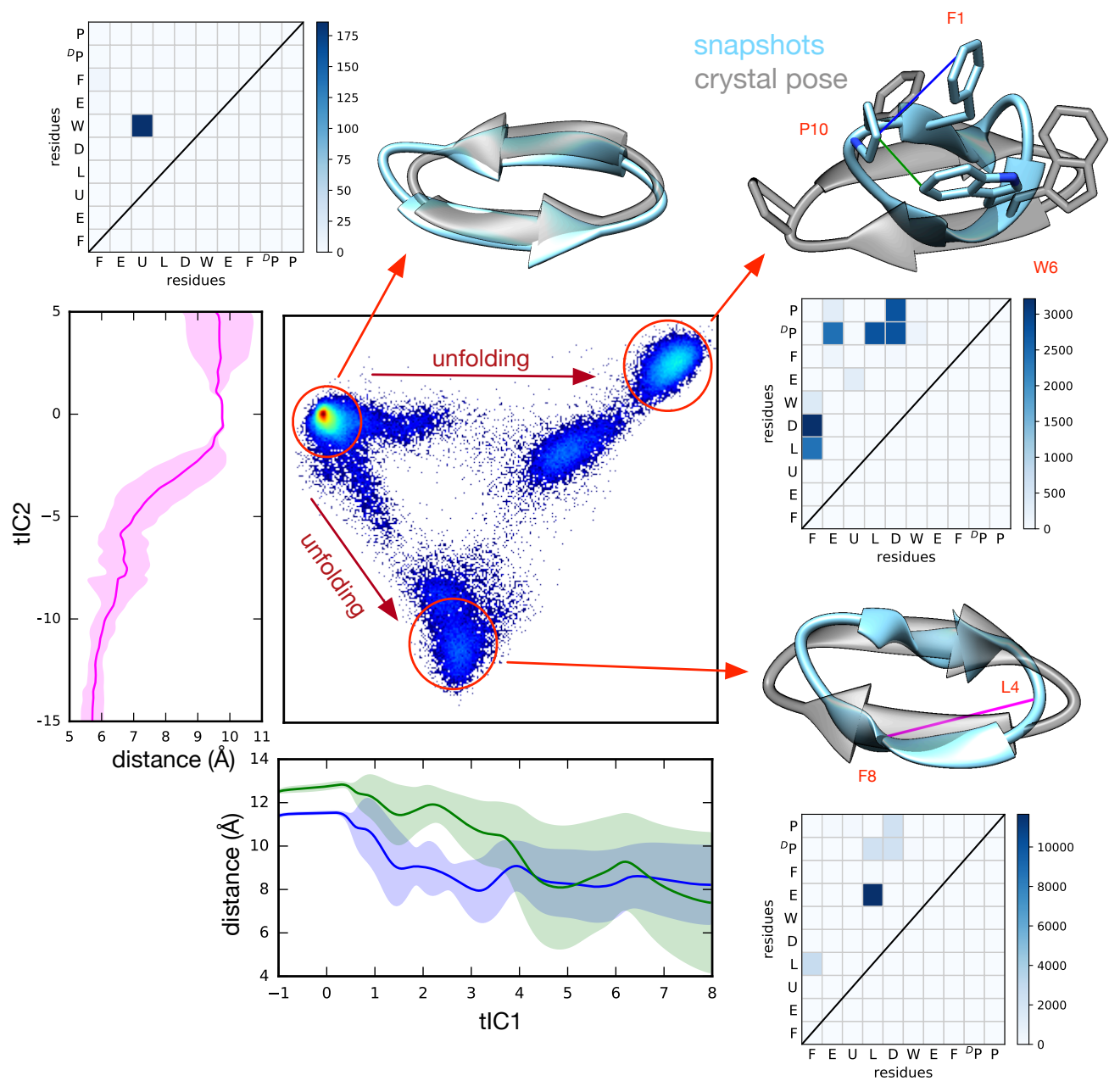

Figure S13: The slowest dynamics (along $\mathrm{tIC}_{1}$ ) of peptide 1 corresponding to a motion that Pro10 and Phe1/Trp6 non-native side chain interactions stabilize the misfolded states. The next slowest motion (along $\mathrm{tIC}_{2}$ ) corresponds to a motion that mainly caused by non-native backbone interactions. The inter-residue distances that change greatly during these motions are shown in blue $\left(\left(\right.\right.$ Phe1- $\left.C^{\zeta}\right)-\left(\right.$ Pro10- $\left.\left.C^{\gamma}\right)\right)$, green $\left(\left(\operatorname{Trp} 6-C^{\zeta 3}\right)\right)-\left(\right.$ Pro10- $\left.\left.C^{\gamma}\right)\right)$, magenta ((Leu4-O)-(Phe8-N)) as a function of $\mathrm{tIC}_{1}$ and $\mathrm{tIC}_{2}$. Contact maps show computed Bayes Factors for all contacts within selected macrostates. Contacts with large Bayes Factor values are those that uniquely define each microstate. In all cases, computed Bayes Factors are consistent with tICA eigenvectors and visual inspection. 


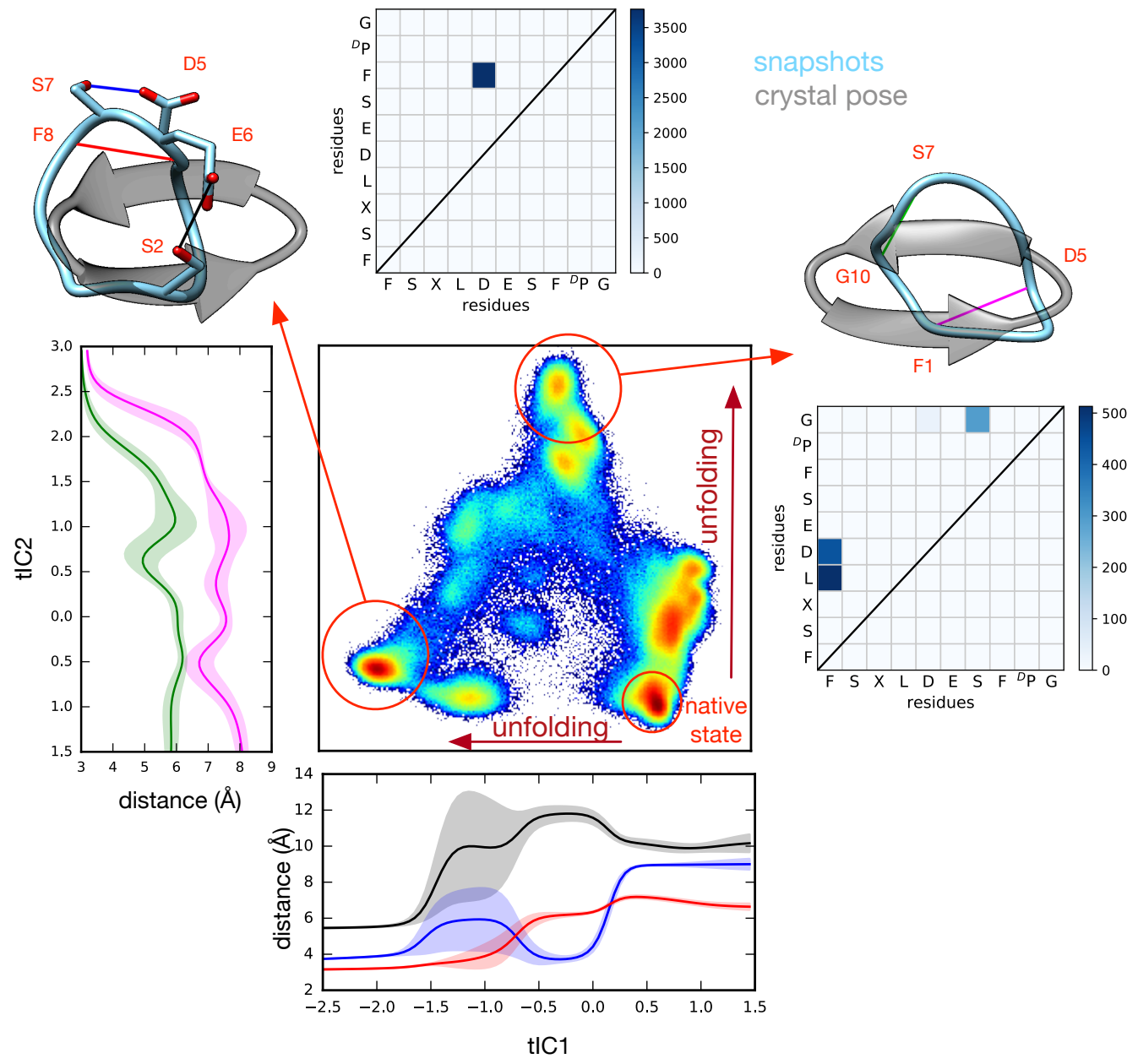

Figure S14: The slowest dynamics (along $\mathrm{tIC}_{1}$ ) of peptide 2 corresponding to a motion that Ser7-Asp5, Phe8-Asp5 and Ser2-Glu6 non-native backbone/side chain interactions stabilize the misfolded states. The next slowest motion (along $\mathrm{tIC}_{2}$ ) corresponds to a motion that mainly caused by non-native backbone interactions. The inter-residue distances that change greatly during these motions are shown in black ((Ser2-O $\left.\left.{ }^{\gamma}\right)-\left(\mathrm{Glu} 6-O^{\epsilon 1}\right)\right)$, blue $((\mathrm{Asp} 5$ $\left.\left.O^{\delta 1}\right)-\left(\operatorname{Ser} 7-O^{\gamma}\right)\right)$, red $\left(\left(\right.\right.$ Asp5-O)- $($ Phe8-N) $)$ as a function of $\mathrm{tIC}_{1}$. The inter-residue distances of (Phe1-O)-(Asp5-N) (magenta) and (Gly10-N)-(Ser7-O) (green) are shown to represent $\mathrm{tIC}_{2}$ motions. Contact maps show computed Bayes Factors for all contacts within selected macrostates. The contact maps do not show some key contacts because only contacts separated by three or more residues are computed. Peptide 3 has identical tICA heatmap and key contacts observed in metastable states as peptide 2 due to identical sequence as peptide 2 (only halogen atoms are different). 


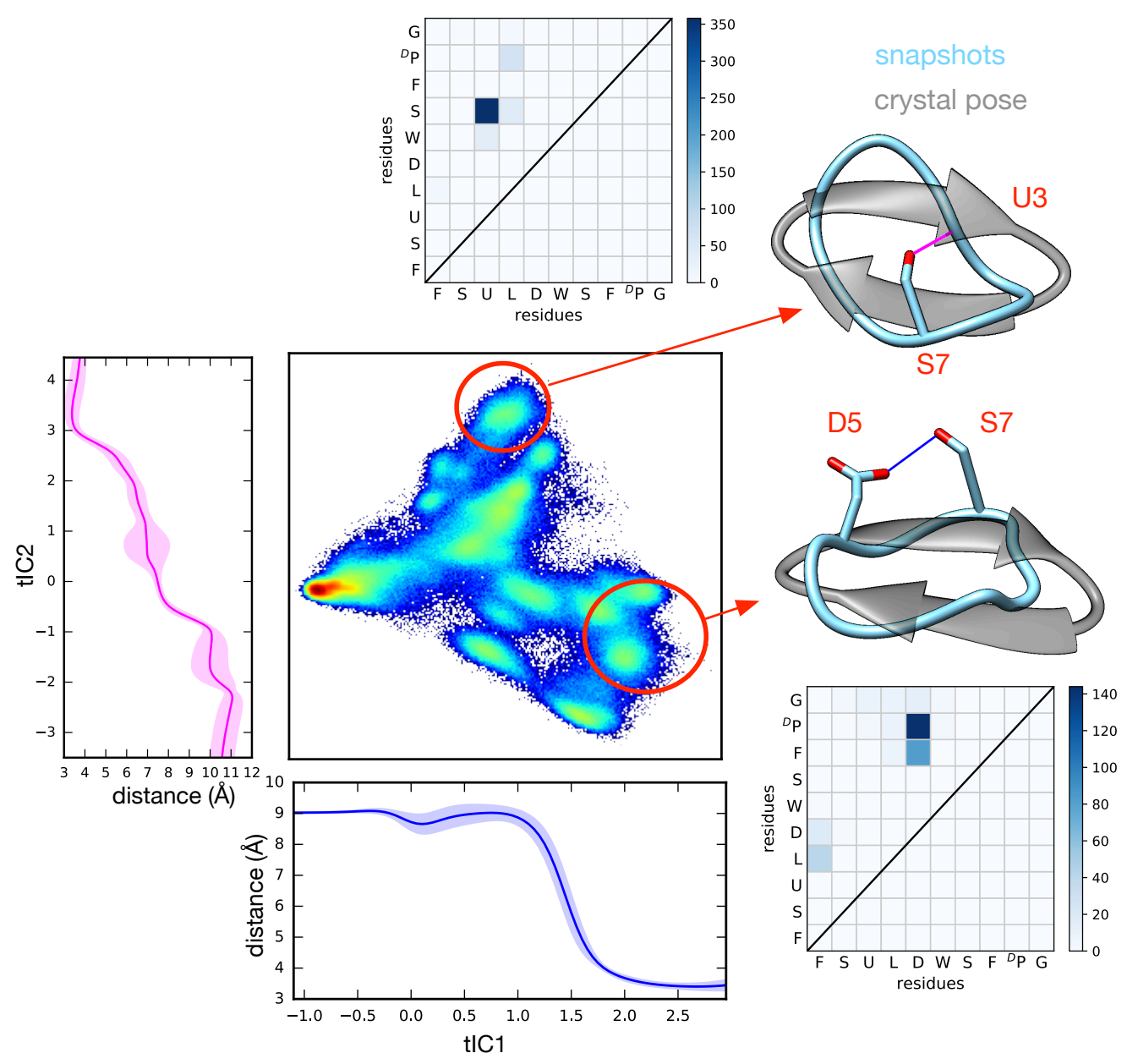

Figure S15: The slowest dynamics (along $\mathrm{tIC}_{1}$ ) of peptide 4 corresponding to a motion that Ser7-Asp5 non-native side chain interactions stabilize the misfolded states. The next slowest motion (along $\mathrm{tIC}_{2}$ ) corresponds to a motion that mainly caused by $\operatorname{Ser} 7-\operatorname{Trp}(\mathrm{Cl}) 3$ non-native interactions. The inter-residue distances that change greatly during these motions are shown in blue $\left(\left(\operatorname{Asp} 5-O^{\delta 1}\right)-\left(\operatorname{Ser} 7-O^{\gamma}\right)\right)$, and magenta $\left((\operatorname{Trp}(\mathrm{Cl}) 3-\mathrm{O})-\left(\operatorname{Ser} 7-O^{\gamma}\right)\right)$ as a function of $\mathrm{tIC}_{1}$ and $\mathrm{tIC}_{2}$, respectively. Contact maps show computed Bayes Factors for all contacts within selected macrostates. The contact maps do not show some key contacts because only contacts separated by three or more residues are computed. 
a

C
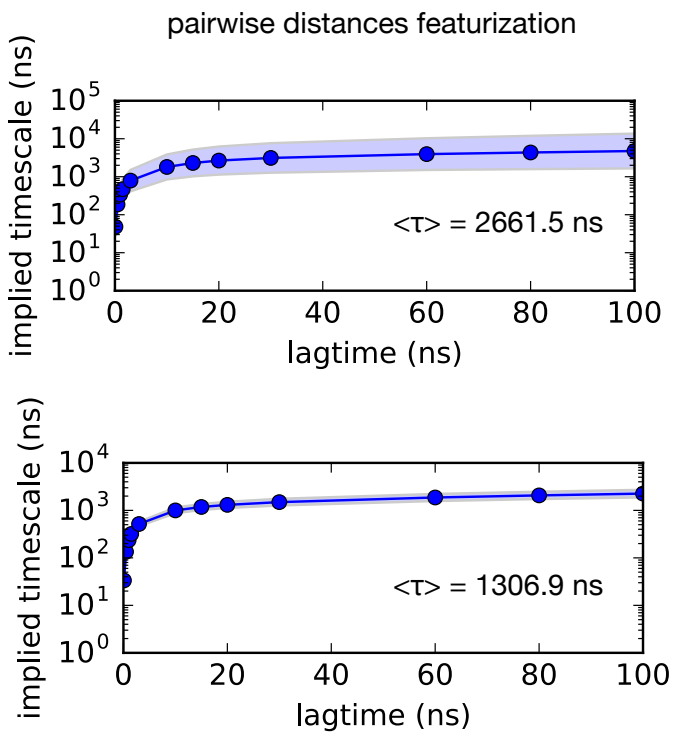

e

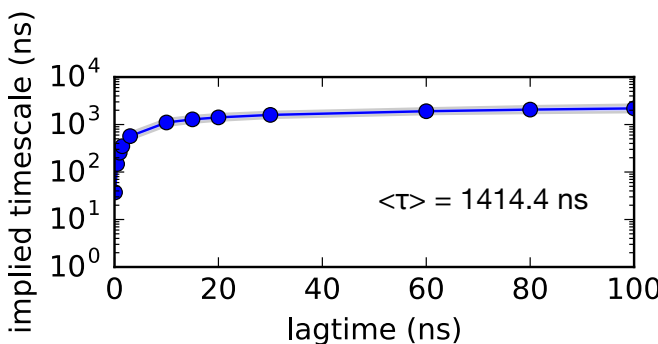

g

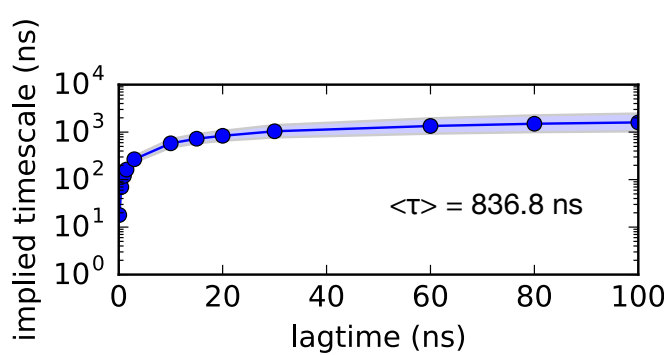

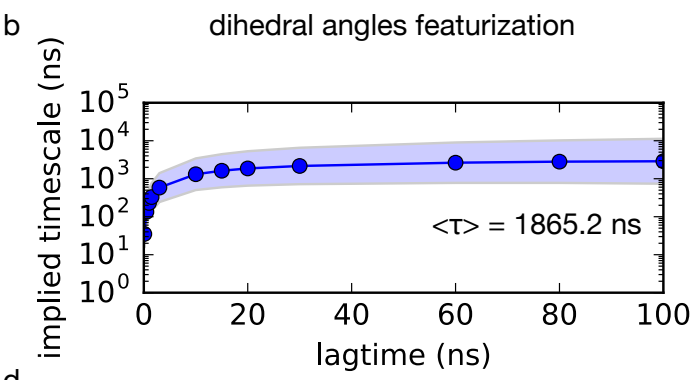

d

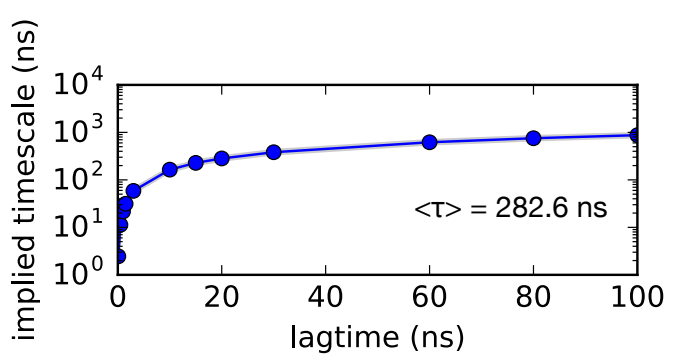

f

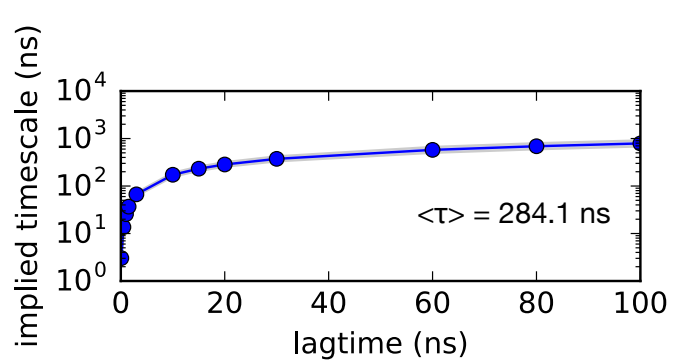

$\mathrm{h}$

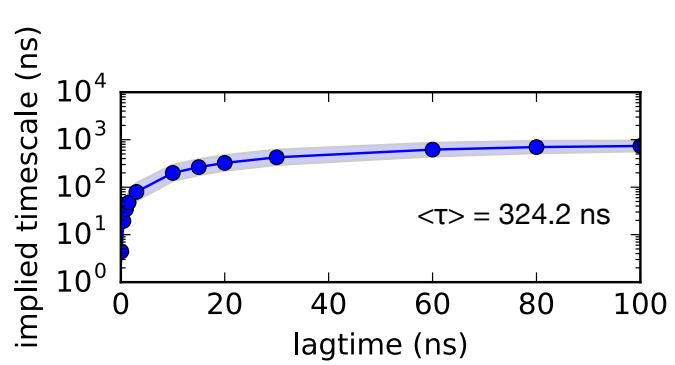

Figure S16: Implied timescale plots for peptide 1-4 using different featurization (pairwise distances: a, c, e, g; dihedral angles: b, d, f, h) in tICA projections. Only the slowest motion timescale is shown with averaged values from a bootstrap procedure, whereby 20 different MSMs were constructed by sampling the input trajectories with replacement. Uncertainty estimates are shown as shaded regions. 

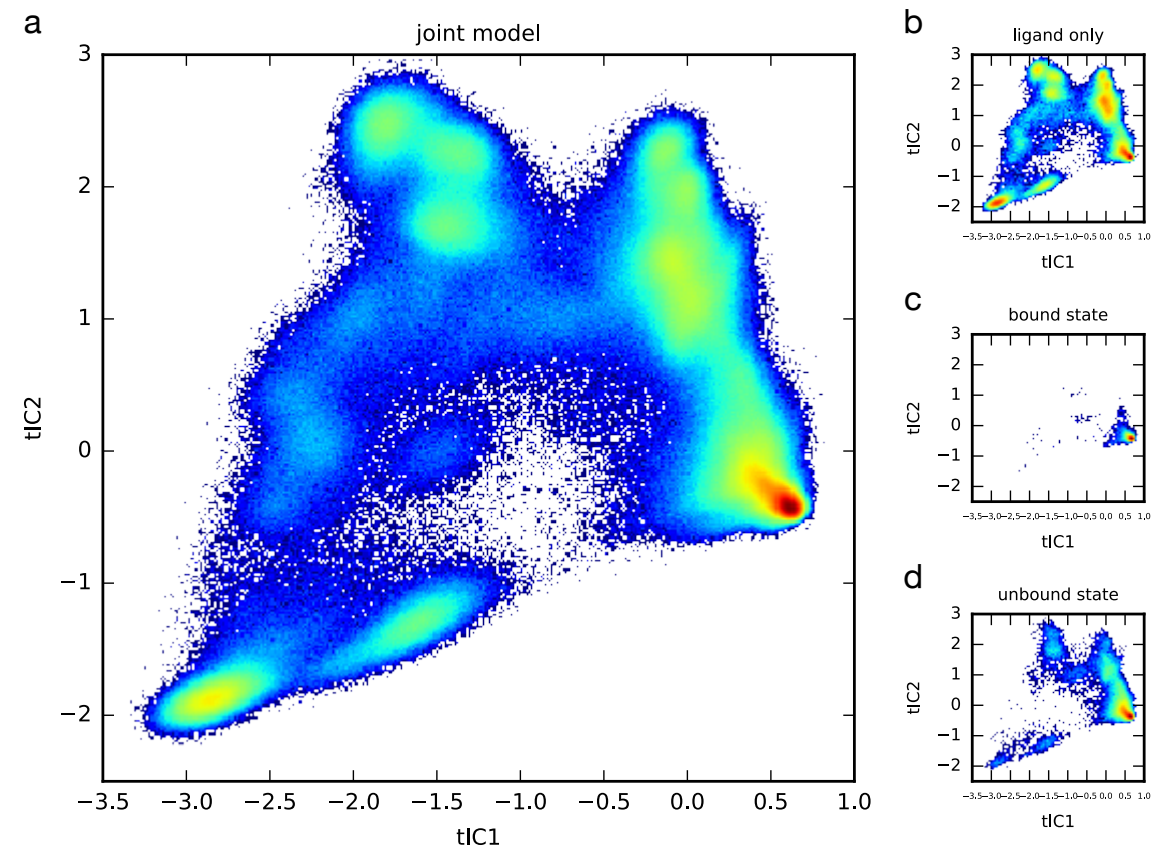

Figure S17: (a) Combined trajectory data of peptide 2 projected to the 2D tICA landscape. (b-d) Transformation of simulation trajectory data using tICA model generated from combined trajectory data for ligand only, bound state and unbound state.
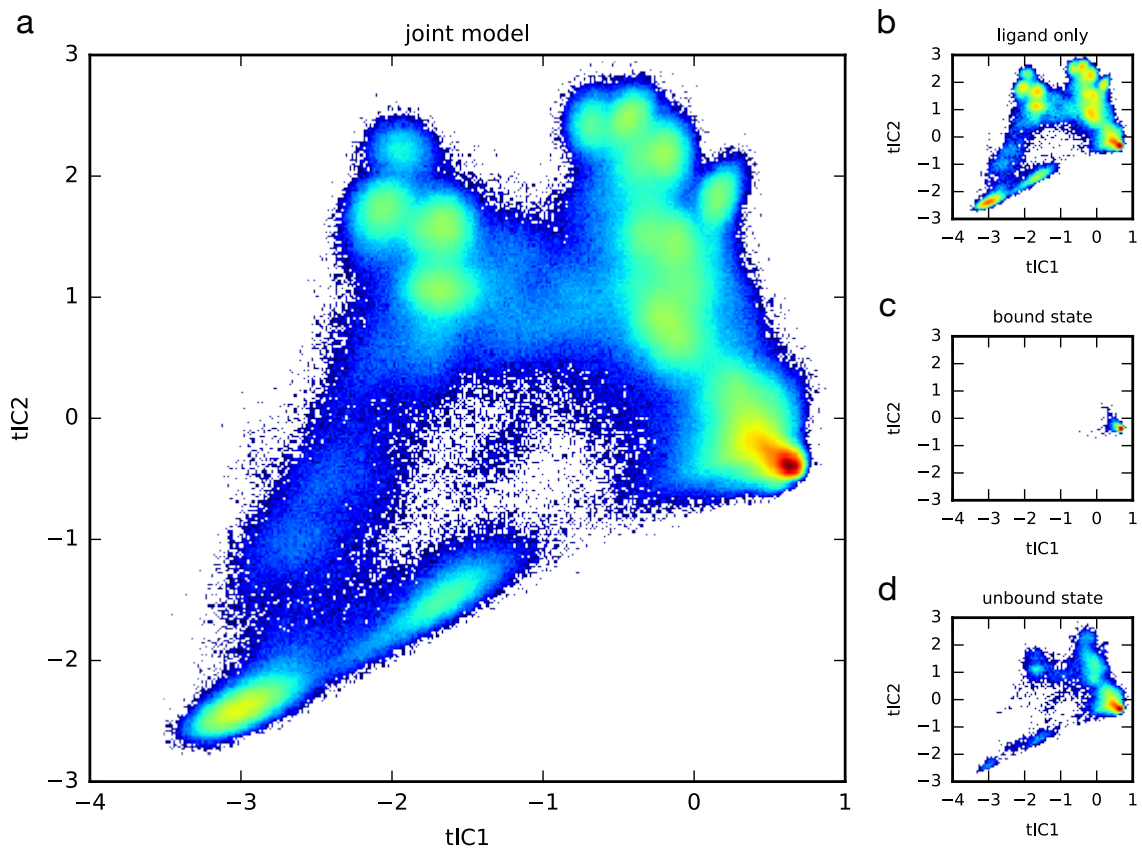

Figure S18: (a) Combined trajectory data of peptide 3 projected to the 2D tICA landscape. (b-d) Transformation of simulation trajectory data using tICA model generated from combined trajectory data for ligand only, bound state and unbound state. 

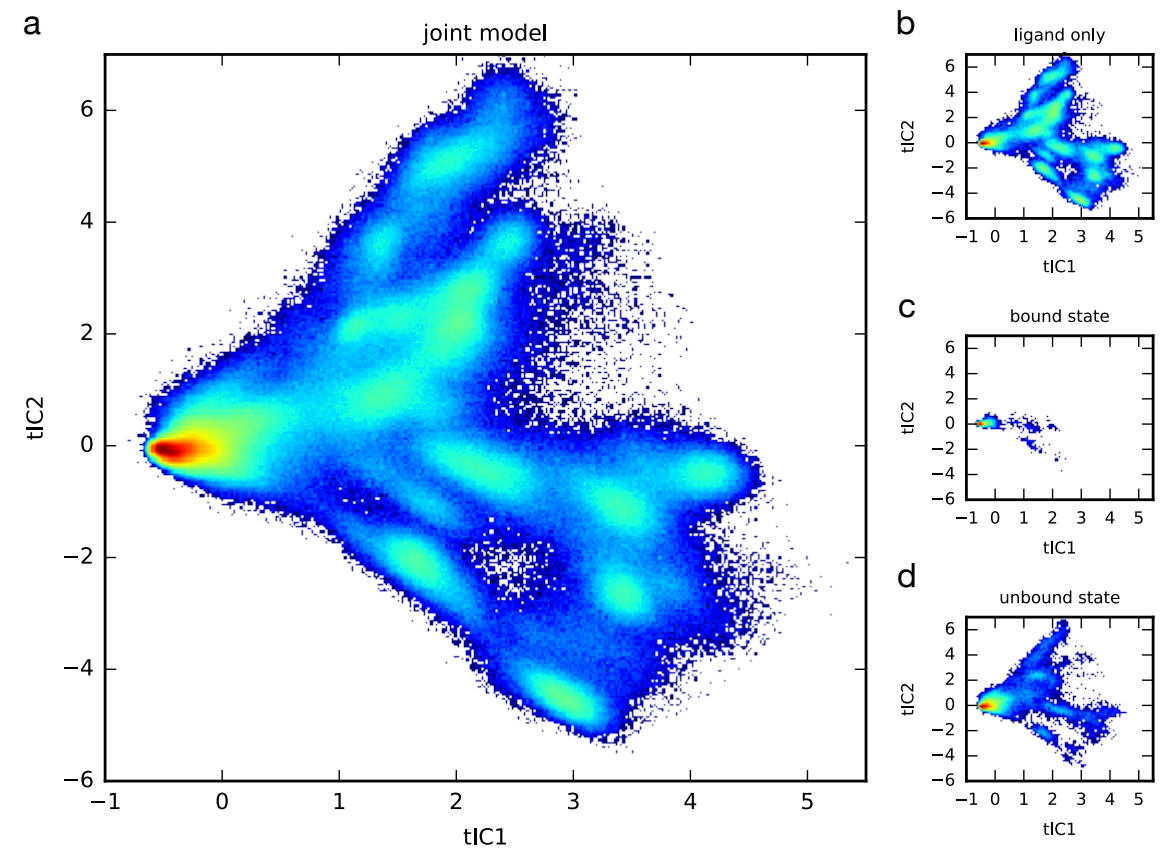

Figure S19: (a) Combined trajectory data of peptide 4 projected to the 2D tICA landscape. (b-d) Transformation of simulation trajectory data using tICA model generated from combined trajectory data for ligand only, bound state and unbound state. 


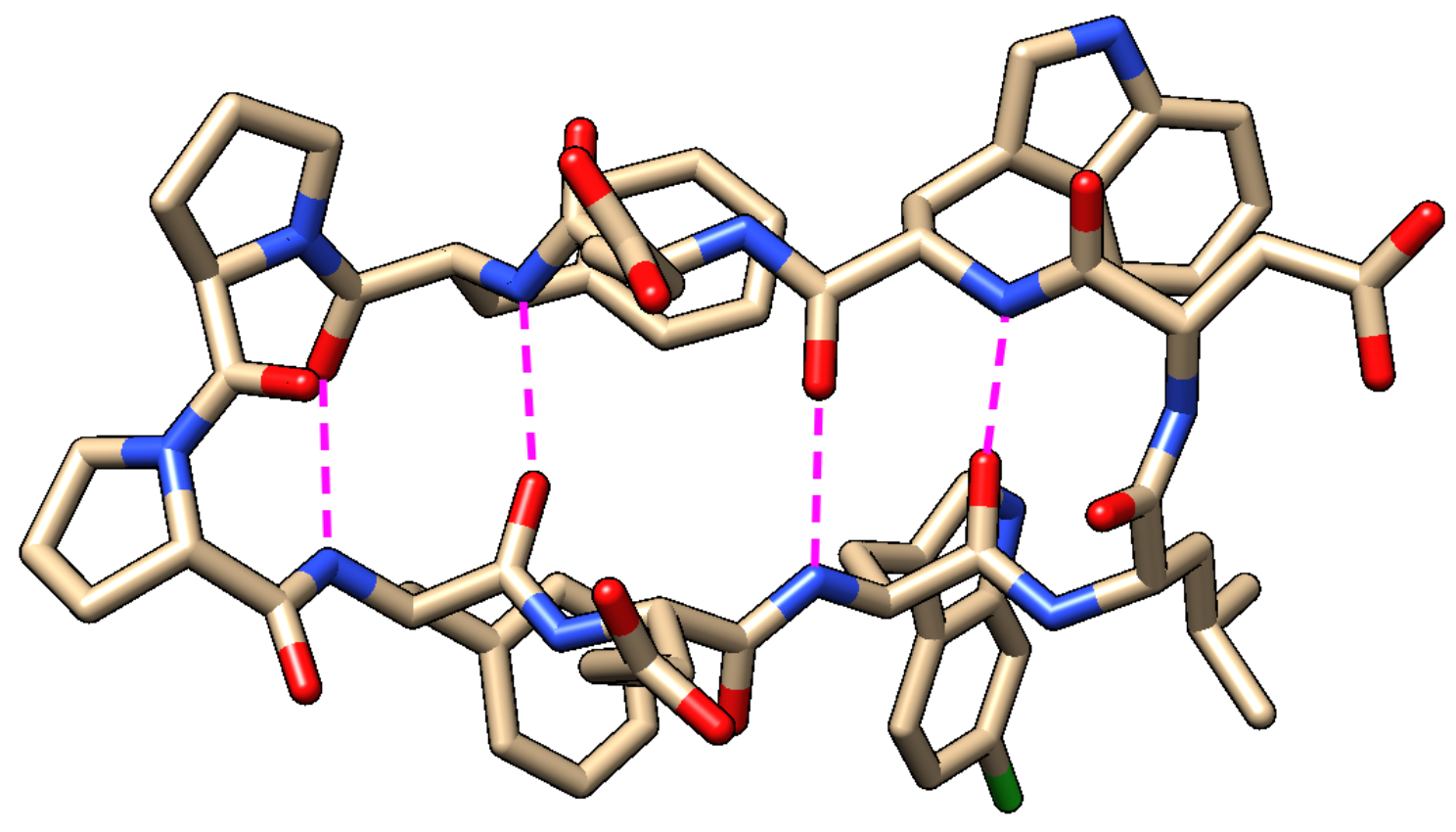

Figure S20: The possible interstrand hydrogen bonds for a folded state. 

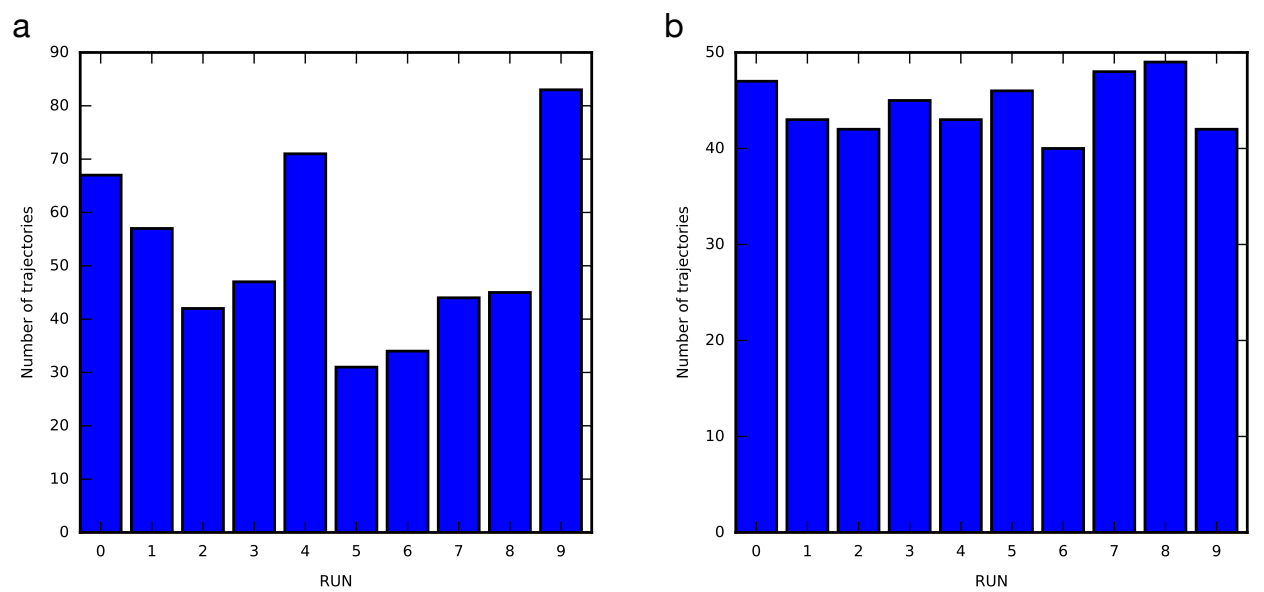

C

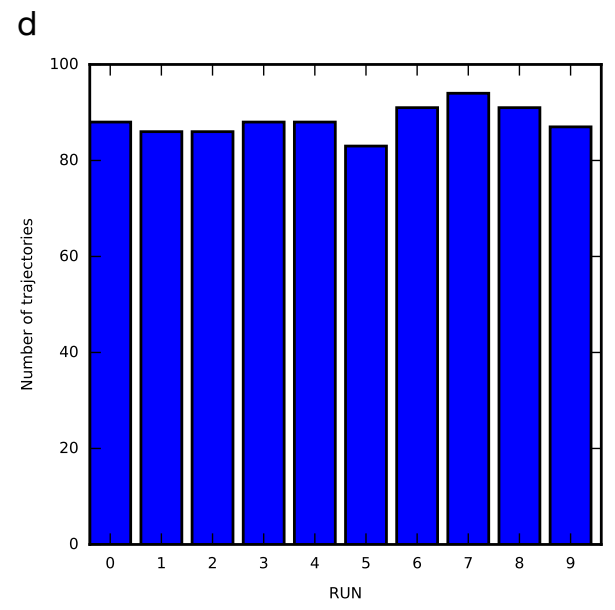

Figure S21: (a-d) Histogram of binding events for initial conformations of simulations (peptide 1-4). This even distribution suggests no bias for any specific initial conformations of simulations. 


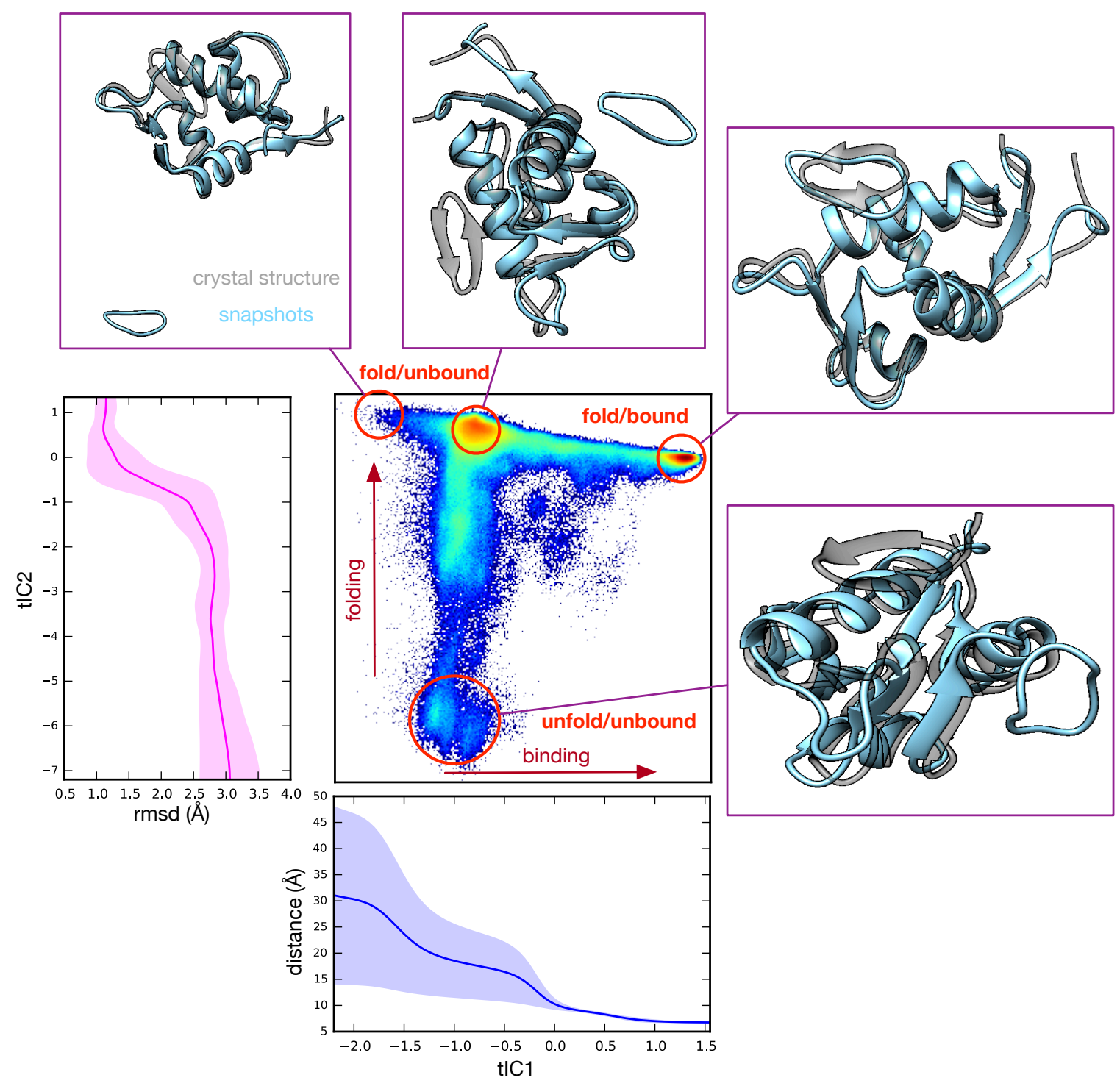

Figure S22: The slowest dynamics (along $\mathrm{tIC}_{1}$ ) of peptide 2 corresponds to the binding process whereas the next slowest dynamics corresponds to the ligand folding process. A conformational selection binding mechanism is revealed from the tICA landscape. Averaged distances between selected residues on MDM2 and the ligand is shown in blue and rmsd change along $\mathrm{tIC}_{2}$ is shown in magenta. Peptide 3 has identical landscape of peptide 2 . 

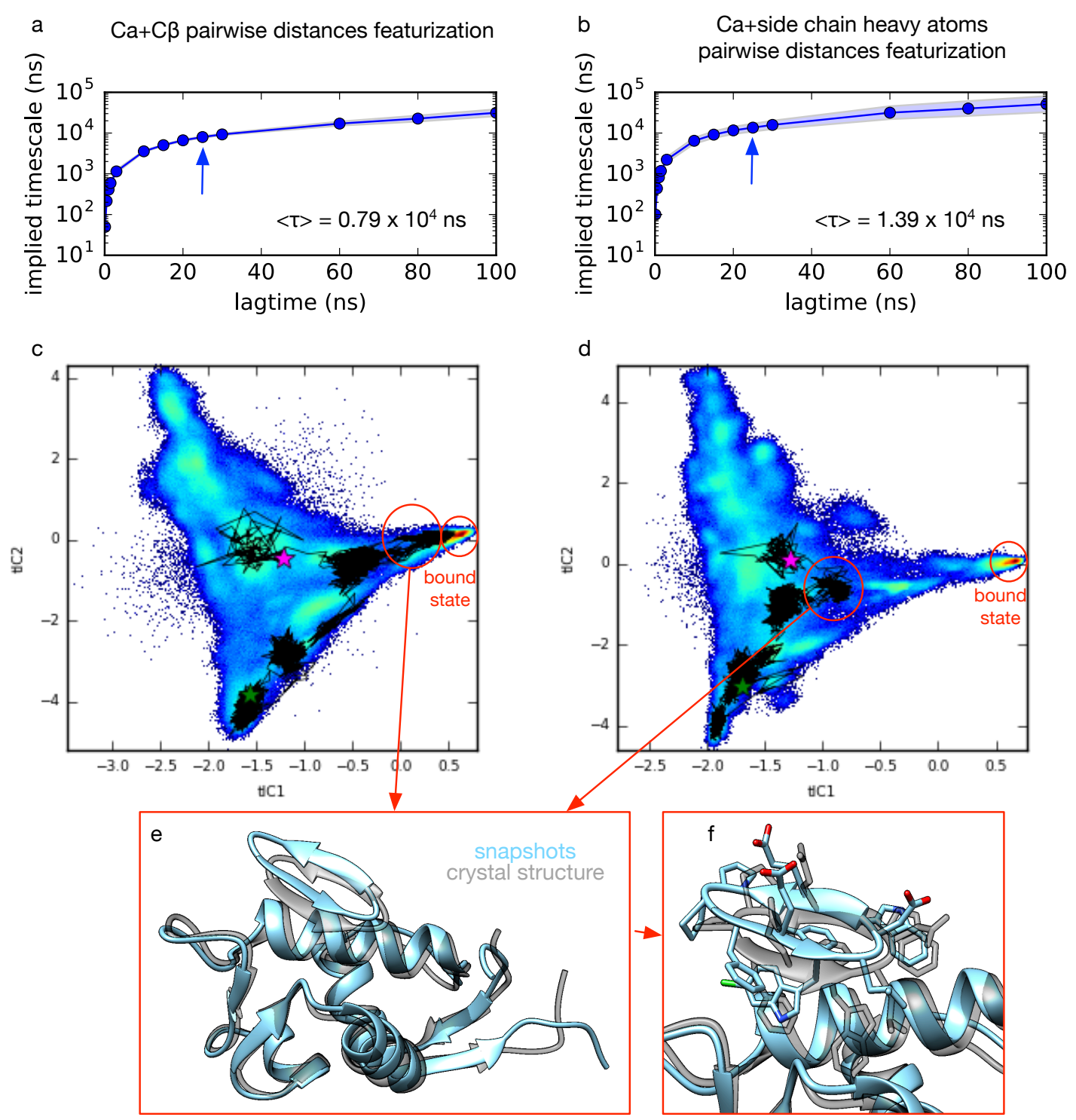

Figure S23: (a-b) Implied timescale plots for peptide 1 using different featurization $(\mathrm{C} \alpha+\mathrm{C} \beta$ pairwise distances and $\mathrm{C} \alpha+$ side chain heavy atoms pairwise distances) in tICA projections. Only the slowest motion timescale is shown with averaged values from a bootstrap procedure, whereby 20 different MSMs were constructed by sampling the input trajectories with replacement. Uncertainty estimates are shown as shaded regions. (c-d) An example trajectory traces shown on tICA heatmap projected using different featurizations. A selected snapshot is shown in (e) with side chain details shown in (f). In the absence of side chain heavy atoms included (c) in featurization, this snapshot is very close to the native bound state but after checking structural details we can see a distinct binding pose of the snapshot from the crystal structure. In the presence of side chain heavy atoms, the traces clearly show a distance from the crystal binding pose for the snapshot which matches the visual inspection. 